\title{
Coding Theorems for Noisy Permutation Channels
}

\author{
Anuran Makur
}

\begin{abstract}
In this paper, we formally define and analyze the class of noisy permutation channels. The noisy permutation channel model constitutes a standard discrete memoryless channel (DMC) followed by an independent random permutation that reorders the output codeword of the DMC. While coding theoretic aspects of this model have been studied extensively, particularly in the context of reliable communication in network settings where packets undergo transpositions, and closely related models of DNA based storage systems have also been analyzed recently, we initiate an information theoretic study of this model by defining an appropriate notion of noisy permutation channel capacity. Specifically, on the achievability front, we prove a lower bound on the noisy permutation channel capacity of any DMC in terms of the rank of the stochastic matrix of the DMC. On the converse front, we establish two upper bounds on the noisy permutation channel capacity of any DMC whose stochastic matrix is strictly positive (entry-wise). Together, these bounds yield coding theorems that characterize the noisy permutation channel capacities of every strictly positive and "full rank" DMC, and our achievability proof yields a conceptually simple, computationally efficient, and capacity achieving coding scheme for such DMCs. Furthermore, we also demonstrate the relation between the wellknown output degradation preorder over channels and noisy permutation channel capacity. In fact, the proof of one of our converse bounds exploits a degradation result that constructs a symmetric channel for any DMC such that the DMC is a degraded version of the symmetric channel. Finally, we illustrate some examples such as the special cases of binary symmetric channels and (general) erasure channels. Somewhat surprisingly, our results suggest that noisy permutation channel capacities are generally quite agnostic to the parameters that define the DMCs.
\end{abstract}

Index Terms-Permutation channel, channel capacity, degradation, second moment method, Doeblin minorization.

\section{CONTENTS}

Introduction

I-A Related Literature and Motivation . . . 2

I-B Noisy Permutation Channel Model . . . 4

I-C Additional Notation ......... 5

I-D Outline ........... 5

II Main Results

II-A Achievability Bound . . . . . . . . 6

II-B Converse Bounds . . . . . . . . . 6

II-C Strictly Positive and Full Rank Channels 6

This work was presented in part at the 2020 IEEE International Symposium on Information Theory [1], and a very preliminary version of this work was presented in part at the 2018 56th Annual Allerton Conference on Communication, Control, and Computing [2].

A. Makur is with the Department of Electrical Engineering and Computer Science, Massachusetts Institute of Technology, Cambridge, MA 02139, USA (e-mail: a_makur@mit.edu).

Copyright (c) 2020 IEEE. Personal use of this material is permitted However, permission to use this material for any other purposes must be obtained from the IEEE by sending a request to pubs-permissions@ieee.org.
II-D Degradation and Noisy Permutation Channel Capacity ......... 6

III Achievability and Converse Bounds 8

III-A Auxiliary Lemmata . . . . . . . . 8

III-B Achievability Bounds for DMCs . . . . 10

III-C Converse Bounds for Strictly Positive DMCs .............. 13

IV Noisy Permutation Channel Capacity 17

IV-A Unit Rank Channels . . . . . . . . . . . 17

IV-B Permutation Transition Matrices . . . . 18

IV-C Strictly Positive Channels . . . . . . . 18

IV-D Erasure Channels and Doeblin Minorization ............ 19

V Conclusion 22

Appendix A: Proof of Proposition 1

Appendix B: Proof of Lemma 4

Appendix C: Proof of Lemma 5 24

Appendix D: Proof of Lemma 6

References $\quad 25$

\section{INTRODUCTION}

In this paper, we initiate an information theoretic study of the problem of reliable communication through noisy permutation channels by defining and analyzing a pertinent notion of information capacity for such channels. Noisy permutation channels refer to discrete memoryless channels (DMCs) followed by independent random permutation transformations that are applied to the entire blocklength of the output codeword. Such channels can be perceived as models of communication links in networks where packets are not delivered in sequence, and hence, the ordering of the packets does not carry any information. Moreover, they also bear a close resemblance to recently introduced models of deoxyribonucleic acid (DNA) based storage systems. The main contributions of this work are the following:

1) We formalize the notion of "noisy permutation channel capacity" of a DMC in Definition 1, which captures, up to first order, the maximum number of messages than can be transmitted through a noisy permutation channel model with vanishing probability of error as the blocklength tends to infinity. (Although our formalism is quite natural, it has not appeared in the literature to our knowledge.) 
2) We establish an achievability bound on the noisy permutation channel capacity of any DMC in terms of the rank of the DMC in Theorem 1 by analyzing a conceptually simple and computationally tractable randomized coding scheme. Moreover, we also demonstrate an alternative proof of our achievability bound for DMCs that have rank 2 in Proposition 2 by using the so called second moment method (in Lemmata 4 and 5).

3) We prove two converse bounds on the noisy permutation channel capacity of any DMC that is strictly positive (entry-wise). The first bound, in Theorem 2, is in terms of the output alphabet size of the DMC, and the second bound, in Theorem 3, is in terms of the "effective input alphabet" size of the DMC. (Neither bound is uniformly better than the other.)

4) Using the aforementioned achievability and converse bounds, we exactly characterize the noisy permutation channel capacity of all strictly positive and "full rank" DMCs in Theorem 4. Furthermore, we propound a candidate solution for the noisy permutation channel capacity of general strictly positive DMCs (regardless of their rank) in Conjecture 1.

5) To complement these results and assist in understanding them, we derive an intuitive monotonicity relation between the degradation preorder over channels and noisy permutation channel capacity in Theorem 5 (also see Theorem 6). Furthermore, we also construct symmetric channels that dominate given DMCs in the degradation sense in Proposition 1. This construction is utilized in the proof of Theorem 3 .

6) Finally, we present exact characterizations of the noisy permutation channel capacities of several specific families of channels, e.g., binary symmetric channels in Proposition 5 (cf. [2, Theorem 3]), channels with unit rank transition kernels in Proposition 3, and channels with permutation matrices as transition kernels in Proposition 4. Furthermore, we present bounds on the noisy permutation channel capacities of (general) erasure channels in Proposition 6, and also propose related conjectures (see, e.g., Conjecture 2). In particular, although Theorem 1 yields our achievability bound for erasure channels, we show an alternative achievability proof in Proposition 6 by exploiting the classical notion of Doeblin minorization.

The ensuing subsections provide some background literature to motivate our study, a formal description of the noisy permutation channel model, some additional notation that will be used throughout the paper, and an outline of the remainder of the paper.

\section{A. Related Literature and Motivation}

The setting of channel coding with transpositions, where the output codeword undergoes some reordering of its symbols, has been widely studied in the coding theory, communication networks, and molecular and biological communications communities. We briefly discuss some relevant literature from these three disciplines, each of which provides a compelling incentive to study noisy permutation channels.
Firstly, in the coding theory literature, one earlier line of work concerned the construction of error-correcting codes that achieve capacity of the random deletion channel, cf. [3]-[5]. The random deletion channel operated on the codeword space by deleting each input codeword symbol independently with some probability $p \in(0,1)$, and copying it otherwise. As expounded in [4, Section I], with sufficiently large alphabet size $2^{b}$, where each symbol of the alphabet was construed as a packet with $b$ bits and $b=\Omega(\log (n))$ depended on the blocklength $n$, "embedding sequence numbers into the transmitted symbols [turned] the deletion channel [into a memoryless] erasure channel." Since coding for erasure channels was well-understood, the intriguing question became to construct (nearly) capacity achieving codes for the random deletion channel using sufficiently large packet length $b$ (depending on $n$ ), but without embedding sequence numbers (see, e.g., [3], [4], and the references therein). ${ }^{1}$ In particular, the author of [4] demonstrated that low density parity check (LDPC) codes with verification-based decoding formed a computationally tractable coding scheme with these properties. Notably, this coding scheme also tolerated transpositions of packets that were not deleted in the process of transmission. Therefore, it was equivalently a coding scheme for a memoryless erasure channel followed by a random permutation block, albeit with an alphabet size that grew polynomially with the blocklength.

Broadly speaking, the results of [3], [4] can be perceived as preliminary steps towards analyzing the fundamental limits of reliable communication through noisy permutation channels where the DMCs are erasure channels. Several other coding schemes for erasure permutation channels with sufficiently large alphabet size have also been developed in the literature. We refer readers to [5], which builds upon the key conceptual ideas in [4], and the references therein for other examples of such coding schemes.

Secondly, this discussion concerning the random deletion channel has a patent counterpart in the (closely related) communication networks literature. Indeed, in the context of the well-known store-and-forward transmission scheme for packet networks, packet losses (or deletions) were typically corrected using Reed-Solomon codes which assumed that each packet carried a header with a sequence number-see, e.g., [7], [8, Section I], and the references therein. Akin to the random deletion channel setting, this simplified the error correction problem since packet losses could be treated as erasures. However, "motivated by networks whose topologies change over time, or where several routes with unequal delays are available to transmit the data," the authors of [8] illustrated that packet errors and losses could also be corrected using binary codes under a channel model where the impaired or lost packets were randomly permuted, and the packets were not indexed with sequence numbers. Such work can also be construed as developing codes for specific kinds of noisy permutation channels.

In general, the noisy permutation channel model in subsection I-B is a simple and useful abstraction for point-to-point

\footnotetext{
${ }^{1}$ We also refer readers to the recent work [6], which proves that Reed-Muller codes achieve capacity for erasure channels, and the references therein.
} 


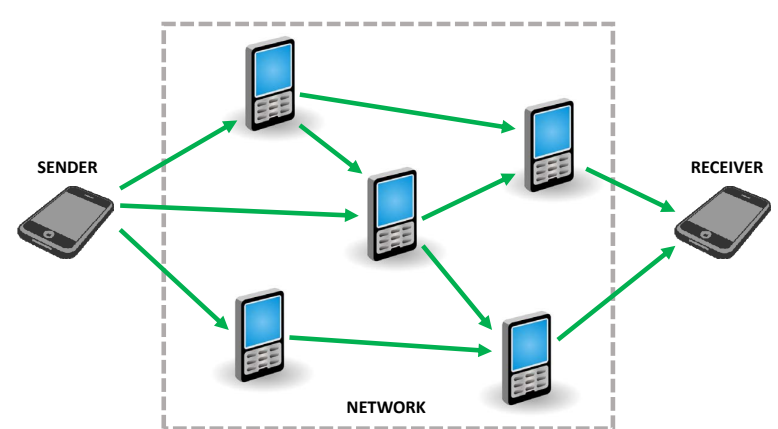

Fig. 1. Illustration of point-to-point communication between a sender and a receiver through a mobile ad hoc network (MANET).

communication between a source and a receiver in various network settings. For instance, when information is transmitted using a lower level multipath routed network (see, e.g., Figure 1 ), the set of all possible packets make up the channel input alphabet with each packet representing a different symbol (as mentioned earlier), and any context specific packet impairments are represented by the DMC in the model. Furthermore, since the packets (or symbols) can take different paths to the receiver in such a network, they may arrive at the destination out-of-order due to different delay profiles in the different paths. This out-of-order delivery of packets is captured by the random permutation transformation in the model. Several other aspects of noisy permutation channels have also been investigated in the communication networks literature. For example, the authors of [9] established rate-delay tradeoffs for multipath routed networks, although they neglected to account for packet impairments, such as deletions, in their analysis for simplicity.

More recently, inspired by packet networks such as mobile ad hoc networks (where the network topology changes over time) - see Figure 1, and heavily loaded datagram-based networks (where packets are often re-routed for load balancing purposes), the authors of [10]-[12] have considered the general problem of coding in channels where the codeword undergoes a random permutation and is subjected to impairments such as insertions, deletions, substitutions, and erasures. As stated in [10, Section I], the basic strategy to reliably communicate across a channel that applies a transformation to its codewords is to "encode the information in an object that is invariant under the given transformation." In the case of noisy permutation channels, the appropriate codes are the so called multiset codes, where the codewords are characterized by their empirical distribution over the underlying alphabet. The existence of certain perfect multiset codes is established in [11], and several other multiset code constructions based on lattices and Sidon sets are analyzed in [12].

Thirdly, an alternative motivation for analyzing noisy permutation channels stems from research at the intersection of computational biology and information theory on DNA based storage systems, cf. [13]-[17]. For example, the authors of [15] examined the storage capacity of systems where the source is encoded using DNA molecules. In their model, source data was encoded into codeword strings (or DNA molecules) consisting of letters from an alphabet of four nucleobases, and short fragments of these codewords were then cached in an unordered fashion akin to the effect of the random permutation in our noisy permutation channel model. The receiver read the encoded data by shotgun sequencing, or equivalently, by randomly sampling the stored and unordered fragments. While the unordered caching aspect of this model resembles our model, as stated in [12, Section I-B], this storage model also differs from our model since the receiver samples the stored codewords with replacement and without errors.

A very closely related DNA based storage model to [15], known as the noisy shuffling channel, is investigated in [17]. Specifically, in order to represent the corruption of DNA molecules during "synthesis, sequencing, and...storage," the authors of [17] studied the storage capacity of a model where DNA codewords first experienced the deleterious effects of a DMC (e.g., a binary symmetric channel), and were then fragmented, and subsequently, the fragments were randomly permuted. (Unlike [15], the receiver had access to all the permuted fragments in this model for simplicity.) The DNA based storage model in [17] is much closer to our noisy permutation channel model than the model in [15]. However, in contrast to our model, both [15] and [17] assume that the lengths of the codeword fragments (which are permuted) grow logarithmically with the number of fragments. We refer readers to [13] for a broader overview of DNA based storage systems, and to [14], [16], and the references therein for other examples of codes for such systems. Moreover, we also refer readers to the comprehensive bibliography in [12] for further related literature on noisy permutation channels.

Finally, it is worth re-emphasizing that the noisy permutation channel model in subsection I-B may be regarded as a variant or generalization of the models described above. More precisely, the analysis in [3], [4] pertains to erasure permutation channels where the alphabet size grows polynomially with the blocklength, the work in [8], [10]-[12] is concerned with various codes for specific noisy permutation channels, and the focus of [17] is on noisy shuffling channels that randomly permute fragments of codewords whose lengths scale logarithmically with the number of fragments. In comparison, our results on noisy permutation channels in this paper consider much broader classes of DMCs, and assume that alphabet sizes are constant with respect to the blocklength, or alternatively, that fragment lengths are constant with respect to the number of fragments. (Note that this latter assumption ensures that we cannot add sequence numbers to packets in order to transform our problem into one of classical coding.)

Furthermore, as the discussion heretofore reveals, the majority of the literature on noisy permutation channels analyzes its coding theoretic aspects. In contrast, we approach these channels from a purely information theoretic perspective. To our knowledge, such a systematic analysis has not been undertaken until now, and thus, there are no known results on the information capacity of the noisy permutation channel model described in the next subsection. (Indeed, while the aforementioned references [3], [15], and [17] have a more information theoretic focus, they analyze different models to 


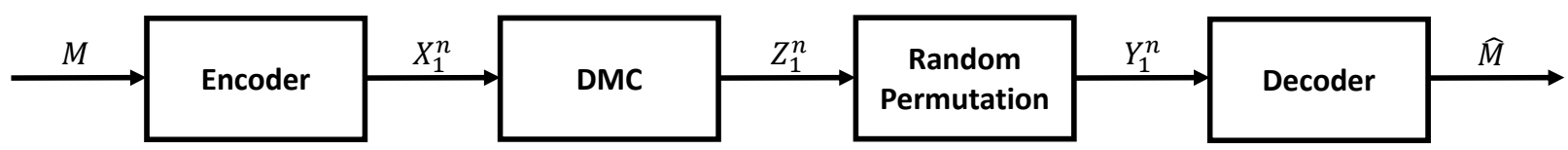

Fig. 2. Illustration of a communication system with a DMC followed by a random permutation.

ours.) In this paper, we will take some first steps towards a complete understanding of the information capacity of noisy permutation channels. Rather interestingly, our main achievability proof will automatically yield computationally tractable codes for reliable communication through certain noisy permutation channels, thereby rendering the need to develop conceptually sophisticated coding schemes for these channels futile when (theoretically) achieving noisy permutation channel capacity is the sole objective.

\section{B. Noisy Permutation Channel Model}

We define the point-to-point noisy permutation channel model in analogy with standard information theoretic definitions, cf. [18, Section 7.5]. Let $n \in \mathbb{N} \triangleq\{1,2,3, \ldots\}$ denote a fixed blocklength, $M \in \mathcal{M} \triangleq\{1, \ldots,|\mathcal{M}|\}$ be a message random variable that is drawn uniformly from the message set $\mathcal{M}, f_{n}: \mathcal{M} \rightarrow \mathcal{X}^{n}$ be a (possibly randomized) encoder, where $\mathcal{X}$ is the finite input alphabet of the channel with $|\mathcal{X}| \geq 2$, and $g_{n}: \mathcal{Y}^{n} \rightarrow \mathcal{M} \cup\{\mathrm{e}\}$ be a (possibly randomized) decoder, where $\mathcal{Y}$ is the finite output alphabet of the channel with $|\mathcal{Y}| \geq 2$ and e denotes an additional "error symbol." The message $M$ is first encoded into a codeword $X_{1}^{n}=f_{n}(M)$, where each $X_{i} \in \mathcal{X}$, and we use the notation $X_{i}^{j} \triangleq\left(X_{i}, \ldots, X_{j}\right)$ for $i<j$. This codeword is transmitted through a (stationary) discrete memoryless channel defined by the conditional probability distributions $\left\{P_{Z \mid X}(\cdot \mid x) \in \mathcal{P}_{\mathcal{Y}}: x \in \mathcal{X}\right\}$ to produce $Z_{1}^{n} \in \mathcal{Y}^{n}$, where each $Z_{i} \in \mathcal{Y}$, and $\mathcal{P} \mathcal{Y}$ denotes the probability simplex in $\mathbb{R}^{|\mathcal{Y}|}$ of all probability distributions on $\mathcal{Y}$. Note that in later sections, we will often treat a DMC $P_{Z \mid X}$ as a row stochastic transition probability matrix $P_{Z \mid X} \in \mathbb{R}^{|\mathcal{X}| \times|\mathcal{Y}|}$ whose rows are given by $\left\{P_{Z \mid X}(\cdot \mid x) \in \mathcal{P}_{\mathcal{Y}}: x \in \mathcal{X}\right\}$, and vice versa, since the two perspectives are equivalent. (In particular, for every $x \in \mathcal{X}$ and $y \in \mathcal{Y}$, the conditional probability $P_{Z \mid X}(y \mid x)$ is also the $(x, y)$ th element of the matrix $P_{Z \mid X}$, i.e., $P_{Z \mid X}(y \mid x)=\left[P_{Z \mid X}\right]_{x, y}$ using the notation in subsection I-C. Likewise, for every $x \in \mathcal{X}$, the conditional distribution $P_{Z \mid X}(\cdot \mid x) \in \mathcal{P}_{\mathcal{Y}}$ forms the $x$ th row of the matrix $\left.P_{Z \mid X}.\right)$ The memorylessness property of the DMC implies that

$$
P_{Z_{1}^{n} \mid X_{1}^{n}}\left(z_{1}^{n} \mid x_{1}^{n}\right)=\prod_{i=1}^{n} P_{Z \mid X}\left(z_{i} \mid x_{i}\right)
$$

for every $x_{1}^{n} \in \mathcal{X}^{n}$ and every $z_{1}^{n} \in \mathcal{Y}^{n}$. The noisy codeword $Z_{1}^{n}$ is then passed through an independent random permutation transformation to generate $Y_{1}^{n} \in \mathcal{Y}^{n}$. Specifically, the random permutation channel $\Pi \triangleq\left\{\Pi\left(\cdot \mid z_{1}^{n}\right)=P_{Y_{1}^{n} \mid Z_{1}^{n}}\left(\cdot \mid z_{1}^{n}\right): z_{1}^{n} \in\right.$ $\left.\mathcal{Y}^{n}\right\}$ is defined as

$$
\begin{aligned}
\Pi\left(y_{1}^{n} \mid z_{1}^{n}\right) & =P_{Y_{1}^{n} \mid Z_{1}^{n}}\left(y_{1}^{n} \mid z_{1}^{n}\right) \\
& =\frac{1}{n !} \sum_{\lambda \in \mathcal{S}_{n}} \mathbb{1}\left\{\forall i \in\{1, \ldots, n\}, y_{\lambda(i)}=z_{i}\right\}
\end{aligned}
$$

for every $y_{1}^{n}, z_{1}^{n} \in \mathcal{Y}^{n}$, where the sum is over all permutations $\lambda:\{1, \ldots, n\} \rightarrow\{1, \ldots, n\}$ in the symmetric group $\mathcal{S}_{n}$ over the set $\{1, \ldots, n\}$, and $\mathbb{1}\{\cdot\}$ is the indicator function defined in subsection I-C. Alternatively, we can describe the action of $\Pi$ in (2) as follows:

1) First, randomly draw a bijection (or permutation) $\lambda$ : $\{1, \ldots, n\} \rightarrow\{1, \ldots, n\}$ uniformly, and independently of everything else, from the symmetric group $\mathcal{S}_{n}$ over $\{1, \ldots, n\}$,

2) Then, generate $Y_{1}^{n}$ from $Z_{1}^{n}$ using the permutation $\lambda$ so that $Y_{\lambda(i)}=Z_{i}$ for all $i \in\{1, \ldots, n\}$.

Throughout this paper, we will refer to random permutation channels on different alphabets, such as the one defined above, as "random permutations" without any further clarification. Finally, the received codeword $Y_{1}^{n}$ is decoded to produce an estimate $\hat{M}=g_{n}\left(Y_{1}^{n}\right)$ of $M$. Figure 2 illustrates this communication system.

Let the average probability of error in this model be

$$
P_{\text {error }}^{(n)} \triangleq \mathbb{P}(M \neq \hat{M}),
$$

where we assume that any decoder $g_{n}$ always makes an error when it outputs the error symbol e. ${ }^{2}$ The "rate" of the encoderdecoder pair $\left(f_{n}, g_{n}\right)$ is defined as

$$
R \triangleq \frac{\log (|\mathcal{M}|)}{\log (n)}
$$

where $\log (\cdot)$ is the binary logarithm (with base 2) throughout this paper, and all Shannon entropy $H(\cdot)$, mutual information $I(\cdot ; \cdot)$, and Kullback-Leibler $(K L)$ divergence (or relative entropy) $D(\cdot \| \cdot)$ terms are measured in bits. ${ }^{3}$ So, we can also write $|\mathcal{M}|=n^{R}$. (Strictly speaking, $n^{R}$ should be an integer, but we will often neglect this detail since it will not affect our results.) We will say that a rate $R \geq 0$ is achievable if there exists a sequence of encoder-decoder pairs $\left\{\left(f_{n}, g_{n}\right)\right\}_{n \in \mathbb{N}}$ such

\footnotetext{
${ }^{2}$ Under an average probability of error criterion, the sequence of encoders $f_{n}$ that minimize $P_{\text {error }}^{(n)}$ are deterministic, and the corresponding sequence of decoders $g_{n}$ that minimize $P_{\text {error }}^{(n)}$ are the maximum a posteriori decoders (or maximum likelihood decoders, since $M$ is uniformly distributed), which are also deterministic without loss of generality [19, Section 16.2.1]. In contrast, under a maximal probability of error criterion, randomized encoders and decoders can be useful [19, Section 16.2.1].

${ }^{3}$ The notion of rate defined in (4) is analogous to the so called third-order coding rate in the finite blocklength analysis literature; see, e.g., [20].
} 
that $\lim _{n \rightarrow \infty} P_{\text {error }}^{(n)}=0$. Lastly, we operationally define the noisy permutation channel capacity as follows.

Definition 1 (Noisy Permutation Channel Capacity). For any DMC $P_{Z \mid X}$, its noisy permutation channel capacity is given by

$$
C_{\text {perm }}\left(P_{Z \mid X}\right) \triangleq \sup \{R \geq 0: R \text { is achievable }\} .
$$

It is straightforward to verify that the scaling in (4) is indeed $\log (n)$ rather than the standard $n$. As mentioned earlier, due to the independent random permutation in the model, all information embedded in the ordering within codewords is lost. (In fact, canonical fixed composition codes cannot carry more than one message in this setting.) So, the maximum number of decodable messages is (intuitively) upper bounded by the number of possible empirical distributions of $Y_{1}^{n}$, i.e.,

$$
n^{R}=|\mathcal{M}| \leq\left(\begin{array}{c}
n+|\mathcal{Y}|-1 \\
|\mathcal{Y}|-1
\end{array}\right) \leq(n+1)^{|\mathcal{Y}|-1},
$$

where taking log's and letting $n \rightarrow \infty$ yields $C_{\text {perm }}\left(P_{Z \mid X}\right) \leq$ $|\mathcal{Y}|-1$ (at least non-rigorously). This justifies that $\log (n)$ is the correct scaling in (4), i.e., the maximum number of messages that can be reliably communicated is polynomial in the blocklength (rather than exponential).

\section{Additional Notation}

In this subsection, we define some additional notation that will be utilized throughout the paper. We begin with some probabilistic notation. The standard expressions $\mathbb{P}(\cdot), \mathbb{E}[\cdot]$, and $\mathbb{V} \mathbb{R}(\cdot)$ represent the probability, expectation, and variance operators, where the underlying probability measures will be clear from context. Moreover, we will write $X \sim P_{X}$ when the random variable $X$ has probability law $P_{X}$. We let $\mathbb{1}\{\cdot\}$ denote the indicator function which equals 1 if its input proposition is true and 0 otherwise. Given any sequence $x_{1}^{n} \in \mathcal{X}^{n}$ with $n \in \mathbb{N}$, we define the empirical distribution (histogram or type) of $x_{1}^{n}$ as

$$
\hat{P}_{x_{1}^{n}}=\left(\hat{P}_{x_{1}^{n}}\left(x^{\prime}\right): x^{\prime} \in \mathcal{X}\right) \in \mathcal{P}_{\mathcal{X}},
$$

where $\hat{P}_{x_{1}^{n}}$ is a probability distribution on $\mathcal{X}$, and for every $x^{\prime} \in \mathcal{X}$,

$$
\hat{P}_{x_{1}^{n}}\left(x^{\prime}\right) \triangleq \frac{1}{n} \sum_{i=1}^{n} \mathbb{1}\left\{x_{i}=x^{\prime}\right\} .
$$

For convenience, we will use the notation

$$
\left(\begin{array}{c}
n \\
n \hat{P}_{x_{1}^{n}}
\end{array}\right) \triangleq \frac{n !}{\prod_{x^{\prime} \in \mathcal{X}}\left(n \hat{P}_{x_{1}^{n}}\left(x^{\prime}\right)\right) !}
$$

for the multinomial coefficient. Furthermore, for any $k \in \mathbb{N}$ and $p \in[0,1]$, we let $\operatorname{Ber}(p)$ denote a Bernoulli distribution with success probability $p$, and $\operatorname{bin}(k, p)$ denote a binomial distribution with $k$ trials and success probability $p$.

Next, we introduce some linear algebraic notation. Fix any $m, n \in \mathbb{N}$. Given any matrix $A \in \mathbb{R}^{m \times n}$, we let $[A]_{i, j}$ denote the $(i, j)$ th element of $A,\|A\|_{\text {op }}$ denote the operator or spectral norm of $A$ (which is the largest singular value of
$A), \sigma_{\min }(A)$ denote the smallest of the $\min \{m, n\}$ singular values of $A, \operatorname{rank}(A)$ denote the rank of $A, A^{\mathrm{T}} \in \mathbb{R}^{n \times m}$ denote the transpose or adjoint of $A, A^{\dagger} \in \mathbb{R}^{n \times m}$ denote the Moore-Penrose pseudoinverse of $A$, and $A^{-1} \in \mathbb{R}^{n \times n}$ denote the inverse of $A$ when $m=n$ and $A$ is non-singular. Furthermore, when the rows of $A$ are linearly independent, then $A^{\dagger}=A^{\mathrm{T}}\left(A A^{\mathrm{T}}\right)^{-1}$ is a right inverse of $A$ such that $A A^{\dagger}=I$, where $I$ is the identity matrix of appropriate dimension. For any row stochastic matrix $A \in \mathbb{R}^{m \times n}$, we let $\operatorname{ext}(A)$ denote the number of extreme points of the convex hull of the rows of $A$, and it is straightforward to verify that

$$
\operatorname{rank}(A) \leq \operatorname{ext}(A) \leq m
$$

In the sequel, we refer to a row stochastic matrix $A$ as full rank if $\operatorname{rank}(A)=\min \{\operatorname{ext}(A), n\},{ }^{4}$ and strictly positive if the elements of $A$ are all strictly positive.

Finally, we present some miscellaneous analysis notation. We let the customary $\|\cdot\|_{p}$ notation denote the $\mathcal{L}^{p}$-norm for $p \in[1, \infty]$. We let $\exp (\cdot)$ denote the natural exponential function (with base $e$ ), and $\lfloor\cdot\rfloor$ denote the floor function. Throughout this paper, we will use the standard BachmannLandau asymptotic notation, e.g., $O(\cdot), \Theta(\cdot), o(\cdot)$, and $\omega(\cdot)$, with the understanding that the parameter $n \rightarrow \infty$ and all other parameters are held constant with respect to $n$.

\section{Outline}

In closing section I, we briefly delineate the organization of the rest of this paper. In section II, we present all of our main results, which were described at the outset of section I. In section III, we prove our main achievability and converse bounds using several auxiliary lemmata. Then, we illustrate several examples of noisy permutation channel capacities for different families of channels in section IV. Furthermore, we also establish the connection between the degradation preorder over channels and noisy permutation channel capacity in section IV. Finally, we conclude our discussion and propose future research directions in section $\mathrm{V}$. On a separate note, it is worth mentioning that throughout this paper, theorems, propositions, and lemmata are stated according to the following convention: If the result is known in the literature, we provide references in the header, and if the result is new, we (obviously) do not provide any references.

\section{MAin Results}

In this section, we present our main results under the setup of subsection I-B, very briefly mention the important ideas in the corresponding proofs, and discuss any related literature where appropriate.

\footnotetext{
${ }^{4}$ This is in contrast to standard usage where $A$ is said to be "full rank" if $\operatorname{rank}(A)=\min \{m, n\}$. Our alternative usage of the phrase "full rank" is motivated by information theoretic contexts, such as in the proof of Theorem 3 in subsection III-C, where the effective number of rows (or input alphabet) of a row stochastic matrix (or channel) $A$ can often be reduced to $\operatorname{ext}(A)$ due to the convexity of KL divergence. The resulting sub-matrix, which has ext $(A)$ rows, is full rank in the standard sense when $\operatorname{rank}(A)=\min \{\operatorname{ext}(A), n\}$.
} 


\section{A. Achievability Bound}

Our first main result is a lower bound on the noisy permutation channel capacity of any DMC in terms of the rank of the DMC.

Theorem 1 (Achievability Bound). The noisy permutation channel capacity of a DMC $P_{Z \mid X}$ is lower bounded by

$$
C_{\text {perm }}\left(P_{Z \mid X}\right) \geq \frac{\operatorname{rank}\left(P_{Z \mid X}\right)-1}{2} .
$$

Theorem 1 is proved in subsection III-B using a simple (randomized) code which enables a basic concentration of measure inequality based argument. We also present an alternative proof of Theorem 1 for the special case of row stochastic matrices with rank 2 in subsection III-B, which employs the so called second moment method for total variation distance.

\section{B. Converse Bounds}

Our second main result is an upper bound on the noisy permutation channel capacity of any strictly positive DMC in terms of the output alphabet size of the DMC.

Theorem 2 (Converse Bound I). The noisy permutation channel capacity of a strictly positive DMC $P_{Z \mid X}$, which means that $P_{Z \mid X}(y \mid x)>0$ for all $x \in \mathcal{X}$ and $y \in \mathcal{Y}$, is upper bounded by

$$
C_{\text {perm }}\left(P_{Z \mid X}\right) \leq \frac{|\mathcal{Y}|-1}{2} .
$$

Theorem 2 is established in subsection III-C. The proof of Theorem 2 uses a Fano's inequality argument followed by a careful application of a central limit theorem (CLT) based approximation of the entropy of a binomial random variable. Intuitively, we also expect to have a converse bound in terms of the input alphabet size, because when $|\mathcal{X}|$ is much smaller than $|\mathcal{Y}|$, there are at most $O\left(n^{|\mathcal{X}|-1}\right)$ distinguishable empirical distributions (rather than $O\left(n^{|\mathcal{Y}|-1}\right)$, as suggested by (5)). Our third main result addresses this intuition by providing an alternative upper bound on the noisy permutation channel capacity of any strictly positive DMC in terms of the number of extreme points of the convex hull of the conditional probability distributions defining the DMC.

Theorem 3 (Converse Bound II). The noisy permutation channel capacity of a strictly positive DMC $P_{Z \mid X}$ is upper bounded by

$$
C_{\text {perm }}\left(P_{Z \mid X}\right) \leq \frac{\operatorname{ext}\left(P_{Z \mid X}\right)-1}{2} .
$$

Theorem 3 is also proved in subsection III-C. Its proof layers a degradation argument, based on Proposition 1 (which will be presented in due course), over the derivation of Theorem 2. We remark that the quantity $\operatorname{ext}\left(P_{Z \mid X}\right)$ can be perceived as an "effective input alphabet" size. Indeed, as elucidated in the proof of Theorem 3, the input alphabet of $P_{Z \mid X}$ can be reduced to a subset of $\mathcal{X}$ corresponding to the extreme points of the convex hull of the rows of $P_{Z \mid X}$ without loss of generality (due, essentially, to the convexity of KL divergence).
Together, the bounds in Theorems 2 and 3 yield the following corollary that for any strictly positive DMC $P_{Z \mid X}$,

$$
C_{\text {perm }}\left(P_{Z \mid X}\right) \leq \frac{\min \left\{\operatorname{ext}\left(P_{Z \mid X}\right),|\mathcal{Y}|\right\}-1}{2} .
$$

On the other hand, for a general DMC $P_{Z \mid X}$, which may have zero entries, we can show that

$$
C_{\text {perm }}\left(P_{Z \mid X}\right) \leq \min \left\{\operatorname{ext}\left(P_{Z \mid X}\right),|\mathcal{Y}|\right\}-1 .
$$

To see this, note that the bound $C_{\text {perm }}\left(P_{Z \mid X}\right) \leq|\mathcal{Y}|-1$ is already intuitively justified by (5), and a rigorous argument follows along the same lines as the converse proof in subsection IV-B. Moreover, the bound $C_{\text {perm }}\left(P_{Z \mid X}\right) \leq \operatorname{ext}\left(P_{Z \mid X}\right)-1$ can be established by following the proof of Theorem 3 in subsection III-C. (Indeed, the derivation of (65) in this proof also holds for DMCs $P_{Z \mid X}$ with zero entries, in which case, $P_{\tilde{Z} \mid \tilde{X}}$ is the identity channel. The converse proof in subsection IV-B can then be applied to yield the desired bound.) We omit these proofs for the sake of brevity.

\section{Strictly Positive and Full Rank Channels}

Theorem 1 and (10) portray that for any strictly positive DMC $P_{Z \mid X}$, the noisy permutation channel capacity satisfies the bounds

$$
\begin{aligned}
\frac{\operatorname{rank}\left(P_{Z \mid X}\right)-1}{2} & \leq C_{\text {perm }}\left(P_{Z \mid X}\right) \\
& \leq \frac{\min \left\{\operatorname{ext}\left(P_{Z \mid X}\right),|\mathcal{Y}|\right\}-1}{2} .
\end{aligned}
$$

Based on the inequalities in (12), we now state (perhaps) the most important result of this paper, which characterizes the noisy permutation channel capacity of the family of strictly positive and full rank channels.

Theorem $4\left(C_{\text {perm }}\right.$ of Strictly Positive and Full Rank Channels). The noisy permutation channel capacity of a strictly positive and full rank DMC $P_{Z \mid X}$ with rank $r \triangleq$ $\operatorname{rank}\left(P_{Z \mid X}\right)=\min \left\{\operatorname{ext}\left(P_{Z \mid X}\right),|\mathcal{Y}|\right\}$ is given by

$$
C_{\text {perm }}\left(P_{Z \mid X}\right)=\frac{r-1}{2} .
$$

Proof. Recalling the definition of "full rank" from subsection I-C, this is an immediate corollary of (12) (i.e., of Theorems 1,2 , and 3 ).

\section{Degradation and Noisy Permutation Channel Capacity}

To complement the aforementioned results, we next present another main result that relates the notion of noisy permutation channel capacity with the so called (output) degradation preorder over channels, which was defined in information theory to study broadcast channels in [21], [22]. (It is worth mentioning that in this paper, we are concerned with the notion of stochastic degradation as opposed to physical degradation, cf. [23, Section 5.4].)

Definition 2 (Degradation Preorder). For any two DMCs (or row stochastic matrices) $P_{Z_{1} \mid X} \in \mathbb{R}^{|\mathcal{X}| \times\left|\mathcal{Z}_{1}\right|}$ and $P_{Z_{2} \mid X} \in$ $\mathbb{R}^{|\mathcal{X}| \times\left|\mathcal{Z}_{2}\right|}$ with common input alphabet $\mathcal{X}$ and output alphabets $\mathcal{Z}_{1}$ and $\mathcal{Z}_{2}$, respectively, we say that $P_{Z_{2} \mid X}$ is a degraded 
version of $P_{Z_{1} \mid X}$ if $P_{Z_{2} \mid X}=P_{Z_{1} \mid X} P_{Z_{2} \mid Z_{1}}$ for some channel $P_{Z_{2} \mid Z_{1}} \in \mathbb{R}^{\left|Z_{1}\right| \times\left|\mathcal{Z}_{2}\right|}$.

The degradation preorder has a long and intriguing history that is worth elaborating on. Its study actually originated in the statistics literature [24]-[26], where it is also known as the Blackwell order. Indeed, the channels $P_{Z_{1} \mid X}$ and $P_{Z_{2} \mid X}$ can be construed as statistical experiments (or observation models) of the parameter space $\mathcal{X}$. In this statistical decision theoretic context, the celebrated Blackwell-Sherman-Stein theorem states that $P_{Z_{2} \mid X}$ is a degraded version of $P_{Z_{1} \mid X}$ if and only if for every prior distribution $P_{X} \in \mathcal{P}_{\mathcal{X}}$, and every real-valued loss function with domain $\mathcal{X} \times \mathcal{X}$, the minimum Bayes risk corresponding to $P_{Z_{1} \mid X}$ is less than or equal to the minimum Bayes risk corresponding to $P_{Z_{2} \mid X}$ [24]-[26] (also see [27] for a simple proof of this result using the separating hyperplane theorem). Furthermore, degradation has beautiful ties with non-Bayesian binary hypothesis testing as well. When $|\mathcal{X}|=2$, the channels $P_{Z_{1} \mid X}$ and $P_{Z_{2} \mid X}$ can be construed as dichotomies of likelihoods, and it can be shown that $P_{Z_{2} \mid X}$ is a degraded version of $P_{Z_{1} \mid X}$ if and only if the Neyman-Pearson function, or receiver operating characteristic curve, of $P_{Z_{1} \mid X}$ dominates the Neyman-Pearson function of $P_{Z_{2} \mid X}$ pointwise (cf. [28, Theorem 5.3] and [29, Section 9.3], where equivalent characterizations using $f$-divergences and majorization are also given). Moreover, for the special case where $P_{Z_{1} \mid X}$ and $P_{Z_{2} \mid X}$ are binary input symmetric channels, other majorization and stochastic domination based characterizations of degradation can be found in [30, Sections 4.1.14-4.1.16]. Finally, we note that degradation is also equivalent to the notion of matrix majorization in [31, Chapter 15, Definition C.8] (also see [32] and [33]). We refer readers to the author's doctoral thesis [34, Section 3.1.1] and [35, Section I-B] for further discussion and references.

The next theorem conveys an intuitive comparison result that if one DMC dominates another DMC in the degradation sense, then the noisy permutation channel capacity of the dominating DMC is larger than the noisy permutation channel capacity of the degraded DMC.

Theorem 5 (Comparison Bound via Degradation). Consider any two DMCs $P_{Z_{1} \mid X} \in \mathbb{R}^{|\mathcal{X}| \times\left|\mathcal{Z}_{1}\right|}$ and $P_{Z_{2} \mid X} \in \mathbb{R}^{|\mathcal{X}| \times\left|\mathcal{Z}_{2}\right|}$, with common input alphabet $\mathcal{X}$ and output alphabets $\mathcal{Z}_{1}$ and $\mathcal{Z}_{2}$, respectively. If $P_{Z_{2} \mid X}$ is a degraded version of $P_{Z_{1} \mid X}$, then we have

$$
C_{\text {perm }}\left(P_{Z_{2} \mid X}\right) \leq C_{\text {perm }}\left(P_{Z_{1} \mid X}\right) .
$$

Theorem 5 is derived in subsection IV-D. As with the setting of traditional channel capacity, the proof of Theorem 5 proceeds by verifying that a noisy permutation channel capacity achieving coding scheme for $P_{Z_{2} \mid X}$ can be used to achieve the same rate and vanishing probability of error when communicating through $P_{Z_{1} \mid X}$. Furthermore, a specialization of Theorem 5 for erasure channels turns out to correspond to the concept of Doeblin minorization, and we use this connection in subsection IV-D to provide an alternative achievability bound on the noisy permutation channel capacity of erasure channels.
Lastly, while we are on the topic of degradation, we present another seemingly disparate result which constructs symmetric channels that dominate given DMCs in the degradation sense. To state this result, we first recall the definition of symmetric channels, cf. [35, Equation (10)].

Definition 3 ( $q$-ary Symmetric Channel). Under the formalism presented in subsection I-B, we define a q-ary symmetric channel with total crossover probability $\delta \in[0,1]$, and input and output alphabet $\mathcal{X}=\mathcal{Y}$ with $|\mathcal{X}|=q \in \mathbb{N} \backslash\{1\}$, denoted $q-\mathrm{SC}(\delta)$, using the doubly stochastic matrix

$$
S_{\delta} \triangleq\left[\begin{array}{ccccc}
1-\delta & \frac{\delta}{q-1} & \cdots & \frac{\delta}{q-1} & \frac{\delta}{q-1} \\
\frac{\delta}{q-1} & 1-\delta & \cdots & \frac{\delta}{q-1} & \frac{\delta}{q-1} \\
\vdots & \vdots & \ddots & \vdots & \vdots \\
\frac{\delta}{q-1} & \frac{\delta}{q-1} & \cdots & 1-\delta & \frac{\delta}{q-1} \\
\frac{\delta}{q-1} & \frac{\delta}{q-1} & \cdots & \frac{\delta}{q-1} & 1-\delta
\end{array}\right] \in \mathbb{R}^{q \times q}
$$

which has $1-\delta$ along its principal diagonal, and $\frac{\delta}{q-1}$ in all other entries. (The rows and columns of $S_{\delta}$ are both indexed consistently by $\mathcal{X}$.)

We note that in the special case where $q=2, \mathcal{X}=\mathcal{Y}=$ $\{0,1\}$, and $\delta$ is the probability that the input bit flips, we refer to the $2-\mathrm{SC}(\delta)$ as a binary symmetric channel (BSC), denoted $\mathrm{BSC}(\delta)$.

The ensuing proposition portrays a sufficient condition for degradation by $q$-ary symmetric channels.

Proposition 1 (Degradation by Symmetric Channels). Suppose we are given a DMC (or row stochastic matrix) $P_{Z \mid X} \in$ $\mathbb{R}^{|\mathcal{X}| \times|\mathcal{Y}|}$ with minimum entry

$$
\nu=\min _{x \in \mathcal{X}, y \in \mathcal{Y}} P_{Z \mid X}(y \mid x),
$$

and a q-ary symmetric channel, $q-\mathrm{SC}(\delta)$, which has a common input alphabet $\mathcal{X}$ such that $|\mathcal{X}|=q$. If the total crossover probability parameter satisfies

$$
0 \leq \delta \leq \frac{\nu}{1-\nu+\frac{\nu}{q-1}},
$$

then $P_{Z \mid X}$ is a degraded version of $q-\mathrm{SC}(\delta)$.

Proposition 1 is proved in appendix A. Although it appears to be unrelated to our thrust towards understanding noisy permutation channel capacity, it turns out to be indispensable in the proof of Theorem 3. We state Proposition 1 here as a main result because we believe it can have many applications in information theory and statistics beyond the context of noisy permutation channels. We refer readers to [35], [36] for further insight regarding the value of studying channel domination by symmetric channels. It is also worth making a few remarks about related results in the literature. Indeed, Proposition 1 establishes a result analogous to [35, Theorem 2] (also see [36, Theorem 2]) that holds for general rectangular row stochastic matrices $P_{Z \mid X}$ (rather than square row stochastic matrices as in [35, Theorem 2]). However, Proposition 1 is weaker than [35, Theorem 2] for square row stochastic matrices (i.e., the upper bound on $\delta$ in [35, Theorem 2] is larger than that in Proposition 1 when $q>2$ ), because the proof of [35, Theorem 


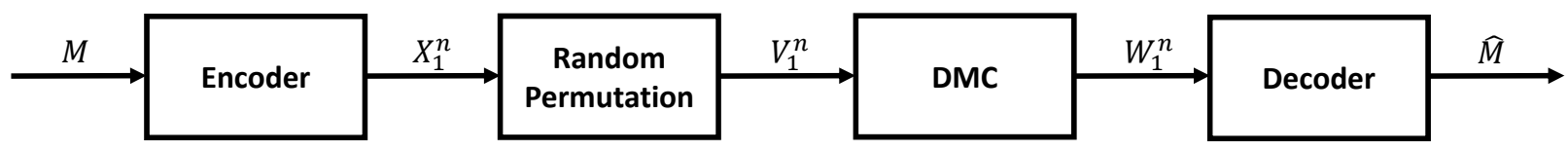

Fig. 3. Illustration of a communication system with a random permutation followed by a DMC.

2] exploits more sophisticated majorization arguments. We also remark that other sufficient conditions for degradation of square row stochastic matrices (or Markov kernels) by $q$-ary symmetric channels, which either use more information than the minimum entries of the matrices (see [37, Proposition 8.1, Equations (8.1) and (8.2)]), or assume further structure on the matrices such as additive noise over Abelian groups (see [35, Theorem 3, Proposition 10] or [36, Theorem 3]), have been derived in the literature.

\section{ACHIEVABILITy AND CONVERse Bounds}

We prove the achievability result in Theorem 1 and the converse results in Theorems 2 and 3 in this section. We commence by presenting some useful lemmata in subsection III-A, and then proceed to establishing the aforementioned theorems in subsections III-B and III-C, respectively.

\section{A. Auxiliary Lemmata}

First, to establish our converse bounds in Theorems 2 and 3, we will present two lemmata. The first lemma we will exploit is the following useful estimate of the entropy of a binomial distribution from the literature.

Lemma 1 (Approximation of Binomial Entropy [38, Equation (7)]). Given a binomial random variable $X \sim \operatorname{bin}(n, p)$ with $n \in \mathbb{N}$ and $p \in(0,1)$, we have

$$
\left|H(X)-\frac{1}{2} \log (2 \pi e n p(1-p))\right| \leq \frac{c(p)}{n}
$$

for some constant $c(p) \geq 0$ (that depends on $p$ ).

The second lemma we will utilize illustrates that swapping the order of the DMC and the random permutation block in the communication system in Figure 2 produces the statistically equivalent communication system in Figure 3.

Lemma 2 (Equivalent Model). Consider the channel $P_{W_{1}^{n} \mid X_{1}^{n}}$ shown in Figure 3, where the input codeword $X_{1}^{n} \in \mathcal{X}^{n}$ passes through an independent random permutation to produce $V_{1}^{n} \in$ $\mathcal{X}^{n}$, and $V_{1}^{n}$ then passes through a DMC $P_{W \mid V}$ to produce the output codeword $W_{1}^{n} \in \mathcal{Y}^{n}$ so that (much like (1))

$\forall v_{1}^{n} \in \mathcal{X}^{n}, w_{1}^{n} \in \mathcal{Y}^{n}, \quad P_{W_{1}^{n} \mid V_{1}^{n}}\left(w_{1}^{n} \mid v_{1}^{n}\right)=\prod_{i=1}^{n} P_{W \mid V}\left(w_{i} \mid v_{i}\right)$.

If the DMC $P_{W \mid V}$ is equal to the DMC $P_{Z \mid X}$ entry-wise, i.e.,

$$
\forall x \in \mathcal{X}, z \in \mathcal{Y}, P_{W \mid V}(z \mid x)=P_{Z \mid X}(z \mid x),
$$

then the channel $P_{W_{1}^{n} \mid X_{1}^{n}}$ is equivalent to the channel $P_{Y_{1}^{n} \mid X_{1}^{n}}$ (described in subsection I-B and Figure 2), i.e.,

$$
\forall x_{1}^{n} \in \mathcal{X}^{n}, y_{1}^{n} \in \mathcal{Y}^{n}, P_{W_{1}^{n} \mid X_{1}^{n}}\left(y_{1}^{n} \mid x_{1}^{n}\right)=P_{Y_{1}^{n} \mid X_{1}^{n}}\left(y_{1}^{n} \mid x_{1}^{n}\right) .
$$

Proof. This follows from direct calculation. Fix any $x_{1}^{n} \in \mathcal{X}^{n}$ and $y_{1}^{n} \in \mathcal{Y}^{n}$. Observe that

$$
\begin{aligned}
& P_{W_{1}^{n} \mid X_{1}^{n}}\left(y_{1}^{n} \mid x_{1}^{n}\right)=\sum_{\substack{v_{1}^{n} \in \mathcal{X}^{n}: \\
\hat{P}_{v_{1}^{n}}=\hat{P}_{x_{1}^{n}}}} P_{W_{1}^{n} \mid V_{1}^{n}}\left(y_{1}^{n} \mid v_{1}^{n}\right) P_{V_{1}^{n} \mid X_{1}^{n}}\left(v_{1}^{n} \mid x_{1}^{n}\right) \\
& =\left(\begin{array}{c}
n \\
n \hat{P}_{x_{1}^{n}}
\end{array}\right)^{-1} \sum_{v_{1}^{n} \in \mathcal{X}^{n}:} \prod_{i=1}^{n} P_{W \mid V}\left(y_{i} \mid v_{i}\right) \\
& \hat{P}_{v_{1}^{n}}^{n}=\hat{P}_{x_{1}^{n}} \\
& =\left(\begin{array}{c}
n \\
n \hat{P}_{x_{1}^{n}}^{n}
\end{array}\right)^{-1} \sum_{\substack{n \\
v_{1}^{n} \in \mathcal{X}^{n}: \\
\hat{P}_{v} n=\hat{P}_{x} n}} \prod_{i=1}^{n} P_{Z \mid X}\left(y_{i} \mid v_{i}\right) \\
& =\left(\begin{array}{c}
n \\
n \hat{P}_{y_{1}^{n}}
\end{array}\right)^{-1}\left(\begin{array}{c}
n \\
n \hat{P}_{x_{1}^{n}}^{n}
\end{array}\right)^{-1} \text {. } \\
& \sum_{\tilde{y}_{1}^{n} \in \mathcal{Y}^{n}:} \sum_{v_{1}^{n} \in \mathcal{X}^{n}:} \prod_{i=1}^{n} P_{Z \mid X}\left(\tilde{y}_{i} \mid v_{i}\right), \\
& \hat{P}_{\tilde{y}_{1}^{n}}=\hat{P}_{y_{1}^{n}} \hat{P}_{v_{1}^{n}}=\hat{P}_{x_{1}^{n}}
\end{aligned}
$$

where the first equality uses the Markov property $X_{1}^{n} \rightarrow$ $V_{1}^{n} \rightarrow W_{1}^{n}$, the third equality follows from (14), and the fourth equality holds because

$$
P_{W_{1}^{n} \mid X_{1}^{n}}\left(y_{1}^{n} \mid x_{1}^{n}\right)=P_{W_{1}^{n} \mid X_{1}^{n}}\left(\tilde{y}_{1}^{n} \mid x_{1}^{n}\right)
$$

for every $\tilde{y}_{1}^{n} \in \mathcal{Y}^{n}$ that is a permutation of $y_{1}^{n}$, which follows from the expression in the third equality. Likewise, we have

$$
\begin{aligned}
& P_{Y_{1}^{n} \mid X_{1}^{n}}\left(y_{1}^{n} \mid x_{1}^{n}\right)=\sum_{z_{1}^{n} \in \mathcal{Y}^{n}:} P_{Y_{1}^{n} \mid Z_{1}^{n}}\left(y_{1}^{n} \mid z_{1}^{n}\right) P_{Z_{1}^{n} \mid X_{1}^{n}}\left(z_{1}^{n} \mid x_{1}^{n}\right) \\
& \hat{P}_{z_{1}^{n}}=\hat{P}_{y_{1}^{n}} \\
& =\left(\begin{array}{c}
n \\
n \hat{P}_{y_{1}^{n}}
\end{array}\right)^{-1} \sum_{\substack{z_{1}^{n} \in \mathcal{Y}^{n}: \\
\hat{P}_{z_{1}^{n}}=\hat{P}_{y_{1}^{n}}}} \prod_{i=1}^{n} P_{Z \mid X}\left(z_{i} \mid x_{i}\right) \\
& =\left(\begin{array}{c}
n \\
n \hat{P}_{x_{1}^{n}}^{n}
\end{array}\right)^{-1}\left(\begin{array}{c}
n \\
n \hat{P}_{y_{1}^{n}}^{n}
\end{array}\right)^{-1} \\
& \sum_{\tilde{x}_{1}^{n} \in \mathcal{X}^{n}:} \sum_{z_{1}^{n} \in \mathcal{Y}^{n}:} \prod_{i=1}^{n} P_{Z \mid X}\left(z_{i} \mid \tilde{x}_{i}\right), \\
& \hat{P}_{\tilde{x}_{1}^{n}}=\hat{P}_{x_{1}^{n}} \hat{P}_{z_{1}^{n}}=\hat{P}_{y_{1}^{n}}
\end{aligned}
$$

where the first equality uses the Markov property $X_{1}^{n} \rightarrow$ $Z_{1}^{n} \rightarrow Y_{1}^{n}$, and the third equality holds because

$$
P_{Y_{1}^{n} \mid X_{1}^{n}}\left(y_{1}^{n} \mid x_{1}^{n}\right)=P_{Y_{1}^{n} \mid X_{1}^{n}}\left(y_{1}^{n} \mid \tilde{x}_{1}^{n}\right)
$$

for every $\tilde{x}_{1}^{n} \in \mathcal{X}^{n}$ that is a permutation of $x_{1}^{n}$, which follows from the expression in the second equality. Therefore, using (15) and (16), we have

$$
P_{W_{1}^{n} \mid X_{1}^{n}}\left(y_{1}^{n} \mid x_{1}^{n}\right)=P_{Y_{1}^{n} \mid X_{1}^{n}}\left(y_{1}^{n} \mid x_{1}^{n}\right),
$$


which completes the proof.

Next, to derive our achievability bound in Theorem 1, we will require the following well-known concentration of measure inequality, which is a specialization of Hoeffding's inequality.

Lemma 3 (Hoeffding's Inequality [39, Theorems 1 and 2]). Suppose $X_{1}, \ldots, X_{n}$ are independent and identically distributed (i.i.d.) random variables such that $\left|X_{1}\right| \leq \sigma$ almost surely for some $\sigma>0$. Then, for every $\gamma \geq 0$,

$$
\mathbb{P}\left(\frac{1}{n} \sum_{i=1}^{n} X_{i}-\mathbb{E}\left[X_{1}\right] \geq \gamma\right) \leq \exp \left(-\frac{n \gamma^{2}}{2 \sigma^{2}}\right)
$$

and

$$
\mathbb{P}\left(\frac{1}{n} \sum_{i=1}^{n} X_{i}-\mathbb{E}\left[X_{1}\right] \leq-\gamma\right) \leq \exp \left(-\frac{n \gamma^{2}}{2 \sigma^{2}}\right) .
$$

While Lemma 3 is used to provide exponentially decaying tail bounds on certain conditional probability of error terms in the proof of Theorem 1 (see (33) and (34) in subsection III-B), we will also show that much weaker tail bounds suffice for proving Theorem 1 for DMCs with rank 2. Indeed, our alternative achievability proof of Proposition 2 in subsection III-B uses the two ensuing lemmata pertaining to the following binary hypothesis testing problem.

Fix any $n \in \mathbb{N}$, and two distinct probability distributions $P_{X}, Q_{X} \in \mathcal{P}_{\mathcal{X}}$ (which can depend on $n$ ). Consider the hypothesis random variable $H \sim \operatorname{Ber}\left(\frac{1}{2}\right)$ (i.e., uniform prior), and likelihoods $P_{X \mid H}(\cdot \mid 0)=P_{X}(\cdot)$ and $P_{X \mid H}(\cdot \mid 1)=Q_{X}(\cdot)$, such that we observe $n$ samples $X_{1}^{n}$ that are drawn conditionally i.i.d. given $H$ from the likelihoods, viz.,

$$
\begin{aligned}
& \text { Given } H=0: X_{1}^{n} \stackrel{\text { i.i.d. }}{\sim} P_{X}, \\
& \text { Given } H=1: X_{1}^{n} \stackrel{\text { i.i.d. }}{\sim} Q_{X} \text {. }
\end{aligned}
$$

The (classical) objective of binary hypothesis testing is to decode the hypothesis $H$ with minimum probability of error from the observed samples $X_{1}^{n}$. It is well-known that the maximum likelihood $(M L)$ decision rule for $H$ based on $X_{1}^{n}$, $\hat{H}_{\mathrm{ML}}^{n}: \mathcal{X}^{n} \rightarrow\{0,1\}$, which is defined by

$$
\forall x_{1}^{n} \in \mathcal{X}^{n}, \hat{H}_{\mathrm{ML}}^{n}\left(x_{1}^{n}\right)=\underset{h \in\{0,1\}}{\arg \max } \prod_{i=1}^{n} P_{X \mid H}\left(x_{i} \mid h\right),
$$

or equivalently,

$$
D\left(\hat{P}_{x_{1}^{n}} \| Q_{X}\right) \quad \stackrel{\hat{H}_{\mathrm{ML}}^{n}\left(x_{1}^{n}\right)=0}{\underset{\hat{H}_{\mathrm{ML}}^{n}\left(x_{1}^{n}\right)=1}{\gtrless}} D\left(\hat{P}_{x_{1}^{n}} \| P_{X}\right),
$$

achieves the minimum probability of error

$$
P_{\mathrm{ML}}^{(n)} \triangleq \mathbb{P}\left(\hat{H}_{\mathrm{ML}}^{n}\left(X_{1}^{n}\right) \neq H\right)
$$

where the tie-breaking rule in (19) (when the likelihoods of 0 and 1 are equal) does not affect $P_{\mathrm{ML}}^{(n)}$ (see, e.g., [40, Chapter 2]). Furthermore, Le Cam's relation states that the ML decoding probability of error is completely characterized by the total variation (TV) distance between the two likelihoods, cf. [41, proof of Theorem 2.2(i)]. Recall that the TV distance between two probability measures $P_{0}$ and $P_{1}$ on a common measurable space $(\mathcal{U}, \mathscr{F})$ is defined as

$$
\begin{aligned}
\left\|P_{0}-P_{1}\right\|_{\mathrm{TV}} & \triangleq \sup _{\mathcal{A} \in \mathscr{F}}\left|P_{0}(\mathcal{A})-P_{1}(\mathcal{A})\right| \\
& =\frac{\left\|P_{0}-P_{1}\right\|_{1}}{2},
\end{aligned}
$$

where (22) is well-known (see, e.g., [42, Chapter 4] for a proof in the discrete case). Then, we have

$$
P_{\mathrm{ML}}^{(n)}=\frac{1}{2}\left(1-\left\|P_{X}^{\otimes n}-Q_{X}^{\otimes n}\right\|_{\mathrm{TV}}\right)
$$

where $P_{X}^{\otimes n}$ and $Q_{X}^{\otimes n}$ denote the $n$-fold product distributions of $X_{1}^{n}$ given $H=0$ and $H=1$, respectively. The next lemma presents a vector generalization of the so called "second moment method for TV distance," cf. [43, Lemma 4.2(iii)], and lower bounds $\left\|P_{X}^{\otimes n}-Q_{X}^{\otimes n}\right\|_{\mathrm{TV}}$.

Lemma 4 (Second Moment Method). For the binary hypothesis testing problem in (17), we have

$$
\left\|P_{X}^{\otimes n}-Q_{X}^{\otimes n}\right\|_{\mathrm{TV}} \geq \frac{\left\|P_{X}-Q_{X}\right\|_{2}^{2}}{4 \sum_{x \in \mathcal{X}} \operatorname{VAR}\left(\hat{P}_{X_{1}^{n}}(x)\right)} .
$$

Lemma 4 is proved in appendix B. Moreover, as mentioned in the remark in appendix B, Lemma 4 can also be construed as a variant of the Hammersley-Chapman-Robbins (HCR) bound in statistics [44], [45].

Our final lemma, Lemma 5, establishes an upper bound on $P_{\mathrm{ML}}^{(n)}$ using Lemma 4. It will be used to derive Proposition 2 in subsection III-B - a specialization of Theorem 1 for DMCs with rank 2 .

Lemma 5 (Testing between Converging Hypotheses). For the binary hypothesis problem in (17), suppose the $\ell^{2}$-distance between $P_{X}$ and $Q_{X}$ is lower bounded by

$$
\left\|P_{X}-Q_{X}\right\|_{2} \geq \frac{1}{n^{\frac{1}{2}-\epsilon_{n}}}
$$

for some constant $\epsilon_{n} \in\left(0, \frac{1}{2}\right)$ (which may depend on $n$ ). Then, we have

$$
P_{\mathrm{ML}}^{(n)} \leq \frac{|\mathcal{X}|}{2|\mathcal{X}|+2 n^{2 \epsilon_{n}}},
$$

which implies that $\lim _{n \rightarrow \infty} P_{\mathrm{ML}}^{(n)}=0$ when $\lim _{n \rightarrow \infty} n^{\epsilon_{n}}=+\infty$.

Lemma 5 is established in appendix C. It illustrates that as long as the Euclidean distance between the likelihoods $P_{X \mid H}(\cdot \mid 0)$ and $P_{X \mid H}(\cdot \mid 1)$ vanishes slower than $\Theta(1 / \sqrt{n})$, we can decode the hypothesis $H$ with vanishing probability of error as $n \rightarrow \infty$. Intuitively, when $\left\|P_{X}-Q_{X}\right\|_{2}=$ $\Theta\left(1 / n^{\frac{1}{2}-\epsilon_{n}}\right)$ and we neglect $\epsilon_{n}$, Lemma 5 holds because the sum of the variances of the entries of the sufficient statistic $T_{n}=\hat{P}_{X_{1}^{n}}-\frac{1}{2} P_{X}-\frac{1}{2} Q_{X}$ (defined in (80) and (85) in the proof of Lemma 4 in appendix B) is $O(1 / n)$. So, as long as the Euclidean distance between the two likelihoods is $\omega(1 / \sqrt{n})$, it is possible to distinguish between the two hypotheses. We also remark that tighter upper bounds on $P_{\mathrm{ML}}^{(n)}$ can be obtained using standard exponential concentration of measure inequalities. However, the simpler second moment method approach will suffice for our proof of Proposition 
2 (while our proof of Theorem 1 will in fact use stronger concentration bounds).

\section{B. Achievability Bounds for DMCs}

In this subsection, we first prove our main achievability result in Theorem 1 and then provide an alternative proof for DMCs with rank 2. Recall the formalism of subsection I-B, which describes the noisy permutation channel model with a DMC $P_{Z \mid X}$.

Proof of Theorem 1. Since the lower bound in Theorem 1 trivially holds for the case $\operatorname{rank}\left(P_{Z \mid X}\right)=1$, we assume without loss of generality that $r \triangleq \operatorname{rank}\left(P_{Z \mid X}\right) \geq 2$. Let $\mathcal{X}^{\prime} \subseteq \mathcal{X}$ denote any (fixed) subset of $\mathcal{X}$ such that $\left|\mathcal{X}^{\prime}\right|=r$ and the set of conditional distributions $\left\{P_{Z \mid X}(\cdot \mid x) \in \mathcal{P}_{\mathcal{Y}}\right.$ : $\left.x \in \mathcal{X}^{\prime}\right\}$ are linearly independent (as vectors in $\mathbb{R}^{|\mathcal{Y}|}$ ), ${ }^{5}$ and let $\tilde{P}_{Z \mid X} \in \mathbb{R}^{r \times|\mathcal{Y}|}$ denote the row stochastic matrix whose rows are given by $\left\{P_{Z \mid X}(\cdot \mid x) \in \mathcal{P}_{\mathcal{Y}}: x \in \mathcal{X}^{\prime}\right\}$. Furthermore, define

$\mathcal{P}_{r, k} \triangleq\left\{\left(\frac{p_{1}}{k}, \ldots, \frac{p_{r}}{k}\right): p_{1}, \ldots, p_{r} \in \mathbb{N} \cup\{0\}, \sum_{i=1}^{r} p_{i}=k\right\}$

as the intersection of the scaled integer lattice $\frac{1}{k} \mathbb{Z}^{r}$ and the probability simplex in $\mathbb{R}^{r}$, where $k \in \mathbb{N}$ is some large constant. Under the setup of subsection I-B, for any $\epsilon \in\left(0, \frac{1}{2}\right)$, consider the following message set and encoder-decoder pair:

1) The message set $\mathcal{M}=\mathcal{P}_{r, k}$ with $k=\left\lfloor n^{\frac{1}{2}-\epsilon}\right\rfloor$ so that the cardinality of $\mathcal{M}$ is

$$
|\mathcal{M}|=\left(\begin{array}{c}
k+r-1 \\
r-1
\end{array}\right)=\Theta\left(n^{\frac{r-1}{2}-\epsilon(r-1)}\right)
$$

where the elements of $\mathcal{M}$ have been re-indexed for convenience.

2) The randomized encoder $f_{n}: \mathcal{P}_{r, k} \rightarrow \mathcal{X}^{n}$ is given by

$$
\forall p=\left(\frac{p_{1}}{k}, \ldots, \frac{p_{r}}{k}\right) \in \mathcal{P}_{r, k}, \quad f_{n}(p)=X_{1}^{n} \stackrel{\text { i.i.d. }}{\sim} P_{X},
$$

where $X_{1}^{n}$ are i.i.d. according to a probability distribution $P_{X} \in \mathcal{P}_{\mathcal{X}}$ such that

$$
P_{X}(x)= \begin{cases}\frac{p_{x}}{k}, & \text { for } x \in \mathcal{X}^{\prime} \\ 0, & \text { for } x \in \mathcal{X} \backslash \mathcal{X}^{\prime}\end{cases}
$$

and $\mathcal{X}^{\prime}=\{1, \ldots, r\}$ without loss of generality.

3) Instead of the ML decoder which achieves minimum probability of error, consider the (sub-optimal) elementwise thresholding decoder $g_{n}: \mathcal{Y}^{n} \rightarrow \mathcal{P}_{r, k} \cup\{\mathrm{e}\}$ defined by

$$
g_{n}\left(y_{1}^{n}\right)= \begin{cases}\left(\frac{\hat{p}_{1}}{k}, \ldots, \frac{\hat{p}_{r}}{k}\right), & \text { if }\left(\frac{\hat{p}_{1}}{k}, \ldots, \frac{\hat{p}_{r}}{k}\right) \in \mathcal{P}_{r, k} \\ \mathrm{e}, & \text { otherwise }\end{cases}
$$

for every $y_{1}^{n} \in \mathcal{Y}^{n}$, where for each $x \in \mathcal{X}^{\prime}$,

$$
\hat{p}_{x}=\underset{j \in\{0, \ldots, k\}}{\arg \min }\left|\sum_{y \in \mathcal{Y}} \hat{P}_{y_{1}^{n}}(y)\left[\tilde{P}_{Z \mid X}^{\dagger}\right]_{y, x}-\frac{j}{k}\right|,
$$

\footnotetext{
${ }^{5}$ This implies that the extreme points of the convex hull of $\left\{P_{Z \mid X}(\cdot \mid x) \in\right.$ $\left.\mathcal{P}_{\mathcal{Y}}: x \in \mathcal{X}^{\prime}\right\}$ are precisely $\left\{P_{Z \mid X}(\cdot \mid x) \in \mathcal{P}_{\mathcal{Y}}: x \in \mathcal{X}^{\prime}\right\}$.
}

where we choose a minimizer randomly when there are several.

This encoder-decoder pair completely specifies the communication system model in subsection I-B. Intuitively, this decoder performs reasonably well because $\tilde{P}_{Z \mid X}^{\dagger}$ is a valid right inverse of $\tilde{P}_{Z \mid X}$ (since the rows of $\tilde{P}_{Z \mid X}$ are linearly independent). Indeed, conditioned on sending a particular message, $\hat{P}_{Y_{1}{ }^{n}}$ is "close" to $P_{Z}$ (which is the true distribution of the $Y_{i}^{1}$ 's as shown below) with high probability when $n$ is large. So, $\sum_{y \in \mathcal{Y}} \hat{P}_{Y_{1}^{n}}(y)\left[\tilde{P}_{Z \mid X}^{\dagger}\right]_{y, x}$ is "close" to the true $P_{X}(x)$ for all $x \in \mathcal{X}^{\prime}$ with high probability. We now analyze the average probability of error for this coding scheme.

Let us condition on the event $\{M=p\}$ for some $p=$ $\left(\frac{p_{1}}{k}, \ldots, \frac{p_{r}}{k}\right) \in \mathcal{P}_{r, k}$. Then, we have

$$
X_{1}^{n} \stackrel{\text { i.i.d. }}{\sim} P_{X}, \quad Z_{1}^{n} \stackrel{\text { i.i.d. }}{\sim} P_{Z}, \quad Y_{1}^{n} \stackrel{\text { i.i.d. }}{\sim} P_{Z},
$$

where $P_{Z}$ denotes the output distribution when $P_{X}$, defined via (28), is "pushed forward" through the channel $P_{Z \mid X}, Z_{1}^{n}$ are i.i.d. because the channel $P_{Z \mid X}$ is memoryless, and $Y_{1}^{n}$ are i.i.d. because they are the output of passing $Z_{1}^{n}$ through an independent random permutation. Let $\mathbb{P}_{p}$ represent the underlying probability measure after conditioning on $\{M=p\}$. The conditional probability that our element-wise thresholding decoder makes an error is upper bounded by

$$
\begin{aligned}
& \mathbb{P}_{p}(\hat{M} \neq M) \\
& =\mathbb{P}_{p}\left(g_{n}\left(Y_{1}^{n}\right) \neq p\right) \\
& =\mathbb{P}_{p}\left(\exists x \in \mathcal{X}^{\prime}, \hat{p}_{x} \neq p_{x}\right) \\
& \stackrel{\text { (a) }}{\leq} \sum_{x \in \mathcal{X}^{\prime}} \mathbb{P}_{p}\left(\hat{p}_{x} \in\{0, \ldots, k\} \backslash\left\{p_{x}\right\}\right) \\
& \stackrel{\text { (b) }}{=} \sum_{x \in \mathcal{X}^{\prime}}\left(\sum_{j>p_{x}} \mathbb{P}_{p}\left(\hat{p}_{x}=j\right)+\sum_{j<p_{x}} \mathbb{P}_{p}\left(\hat{p}_{x}=j\right)\right) \\
& \stackrel{(\text { c) })}{\leq} \sum_{x \in \mathcal{X}^{\prime}} \sum_{j>p_{x}} \mathbb{P}_{p}\left(\sum_{y \in \mathcal{Y}} \hat{P}_{Y_{1}^{n}}(y)\left[\tilde{P}_{Z \mid X}^{\dagger}\right]_{y, x} \geq \frac{p_{x}+j}{2 k}\right) \\
& \quad+\sum_{x \in \mathcal{X}^{\prime}} \sum_{j<p_{x}} \mathbb{P}_{p}\left(\sum_{y \in \mathcal{Y}} \hat{P}_{Y_{1}^{n}}(y)\left[\tilde{P}_{Z \mid X}^{\dagger}\right]_{y, x} \leq \frac{p_{x}+j}{2 k}\right),
\end{aligned}
$$

where (a) follows from the union bound, (b) splits a summation over $j \in\{0, \ldots, k\} \backslash\left\{p_{x}\right\}$ into two summations (and one of these summations is 0 if $p_{x} \in\{0, k\}$ ), and (c) holds because $\hat{p}_{x}=j$ implies that $k \sum_{y \in \mathcal{Y}} \hat{P}_{Y_{1}^{n}}(y)\left[\tilde{P}_{Z \mid X}^{\dagger}\right]_{y, x}$ is closer to $j$ than $p_{x}$ due to (30), and we count the tie case, where $k \sum_{y \in \mathcal{Y}} \hat{P}_{Y_{1}^{n}}(y)\left[\tilde{P}_{Z \mid X}^{\dagger}\right]_{y, x}$ is equally close to $j$ and $p_{x}$, as an error since this gives us an upper bound on the desired conditional probability of error.

To show that this upper bound in (31) vanishes, observe that 
for any $x \in \mathcal{X}^{\prime}$ and any $j>p_{x}$ (assuming $p_{x}<k$ ),

$$
\begin{aligned}
\mathbb{P}_{p}\left(\sum_{y \in \mathcal{Y}} \hat{P}_{Y_{1}^{n}}(y)\left[\tilde{P}_{Z \mid X}^{\dagger}\right]_{y, x} \geq \frac{p_{x}+j}{2 k}\right) \\
\quad=\mathbb{P}_{p}\left(\frac{1}{n} \sum_{i=1}^{n}\left[\tilde{P}_{Z \mid X}^{\dagger}\right]_{Y_{i}, x}-\frac{p_{x}}{k} \geq \frac{j-p_{x}}{2 k}\right),
\end{aligned}
$$

which holds because

$$
\sum_{y \in \mathcal{Y}} \hat{P}_{Y_{1}^{n}}(y)\left[\tilde{P}_{Z \mid X}^{\dagger}\right]_{y, x}=\frac{1}{n} \sum_{i=1}^{n}\left[\tilde{P}_{Z \mid X}^{\dagger}\right]_{Y_{i}, x}
$$

almost surely. To bound the right hand side of (32), we notice three facts:

1) $\left\{\left[\tilde{P}_{Z \mid X}^{\dagger}\right]_{Y_{i}, x}: i \in\{1, \ldots, n\}\right\}$ are i.i.d. random variables, because $\left[\tilde{P}_{Z \mid X}^{\dagger}\right]_{Y_{i}, x}$ is a deterministic function of $Y_{i}$ (since $\tilde{P}_{Z \mid X}^{\dagger}$ is a known deterministic matrix), and $Y_{1}^{n}$ are i.i.d. random variables given $M=p$.

2) For each $i \in\{1, \ldots, n\},\left[\tilde{P}_{Z \mid X}^{\dagger}\right]_{Y_{i}, x}$ is bounded almost surely by

$$
\begin{aligned}
\left|\left[\tilde{P}_{Z \mid X}^{\dagger}\right]_{Y_{i}, x}\right| & =\left|e_{Y_{i}}^{\mathrm{T}} \tilde{P}_{Z \mid X}^{\dagger} e_{x}\right| \\
& \leq \max _{\substack{u \in \mathbb{R}^{|\mathcal{Y}|}, v \in \mathbb{R}^{r}: \\
\|u\|_{2}=\|v\|_{2}=1}}\left|u^{\mathrm{T}} \tilde{P}_{Z \mid X}^{\dagger} v\right| \\
& =\left\|\tilde{P}_{Z \mid X}^{\dagger}\right\|_{\text {op }} \triangleq \sigma,
\end{aligned}
$$

where $e_{j}$ denotes the $j$ th standard basis vector with unity at the $j$ th position and zero elsewhere, and the last equality follows from the Courant-Fischer-Weyl minmax theorem, cf. [46, Section 3.1, Problem 6, p.155], [47, Lemma 2].

3) For each $i \in\{1, \ldots, n\},\left[\tilde{P}_{Z \mid X}^{\dagger}\right]_{Y_{i}, x}$ has expected value

$$
\begin{aligned}
\mathbb{E}_{p}\left[\left[\tilde{P}_{Z \mid X}^{\dagger}\right]_{Y_{i}, x}\right] & =\sum_{y \in \mathcal{Y}} \mathbb{E}_{p}\left[\hat{P}_{Y_{1}^{n}}(y)\right]\left[\tilde{P}_{Z \mid X}^{\dagger}\right]_{y, x} \\
& =\sum_{y \in \mathcal{Y}} P_{Z}(y)\left[\tilde{P}_{Z \mid X}^{\dagger}\right]_{y, x} \\
& =P_{X}(x) \\
& =\frac{p_{x}}{k}
\end{aligned}
$$

where $\mathbb{E}_{p}[\cdot]$ represents expectation with respect to the conditional probability distribution of $Y_{1}^{n}$ given $M=p$, the third equality crucially uses the fact that $P_{Z \mid X}$ has a right inverse since its rows are linearly independent, ${ }^{6}$ and the final equality follows from (28).

Using these facts, we can apply Lemma 3 to the right hand side of (32) and obtain

$$
\begin{aligned}
\mathbb{P}_{p}\left(\sum_{y \in \mathcal{Y}} \hat{P}_{Y_{1}^{n}}(y)\left[\tilde{P}_{Z \mid X}^{\dagger}\right]_{y, x}\right. & \left.\geq \frac{p_{x}+j}{2 k}\right) \\
\leq & \exp \left(-\frac{n\left(j-p_{x}\right)^{2}}{8 \sigma^{2} k^{2}}\right)
\end{aligned}
$$

\footnotetext{
${ }^{6}$ The existence of a right inverse of $P_{Z \mid X}$ ensures that the input distribution $P_{X}$ can be uniquely recovered from the output distribution $P_{Z}$ and $P_{Z \mid X}$. This elucidates why our achievability bound depends on the rank of $P_{Z \mid X}$.
}

for any $x \in \mathcal{X}^{\prime}$ and any $j>p_{x}$ (assuming $p_{x}<k$ ). Likewise, for any $x \in \mathcal{X}^{\prime}$ and any $j<p_{x}$ (assuming $p_{x}>0$ ), Lemma 3 yields

$$
\begin{aligned}
\mathbb{P}_{p}\left(\sum_{y \in \mathcal{Y}} \hat{P}_{Y_{1}^{n}}(y)\left[\tilde{P}_{Z \mid X}^{\dagger}\right]_{y, x}\right. & \left.\leq \frac{p_{x}+j}{2 k}\right) \\
\leq & \exp \left(-\frac{n\left(j-p_{x}\right)^{2}}{8 \sigma^{2} k^{2}}\right) .
\end{aligned}
$$

So, bounding the terms in (31) using (33) and (34) produces

$$
\begin{aligned}
\mathbb{P}_{p}(\hat{M} \neq M) & \leq \sum_{x \in \mathcal{X}^{\prime}} \sum_{j \in\{0, \ldots, k\} \backslash\left\{p_{x}\right\}} \exp \left(-\frac{n\left(j-p_{x}\right)^{2}}{8 \sigma^{2} k^{2}}\right) \\
& \leq \sum_{x \in \mathcal{X}^{\prime}} \sum_{j \in\{0, \ldots, k\} \backslash\left\{p_{x}\right\}} \exp \left(-\frac{n}{8 \sigma^{2} n^{1-2 \epsilon}}\right) \\
& \leq r n^{\frac{1}{2}-\epsilon} \exp \left(-\frac{n^{2 \epsilon}}{8 \sigma^{2}}\right)
\end{aligned}
$$

where the second inequality holds because $k=\left\lfloor n^{\frac{1}{2}-\epsilon}\right\rfloor \leq$ $n^{\frac{1}{2}-\epsilon}$ and $\left|j-p_{x}\right| \geq 1$ for all $x \in \mathcal{X}^{\prime}$ and all $j \neq p_{x}$, and the third inequality holds because $\left|\mathcal{X}^{\prime}\right|=r$ and $k \leq n^{\frac{1}{2}-\epsilon}$.

Lastly, taking expectations with respect to the law of $M$ in (35) yields

$$
P_{\text {error }}^{(n)} \leq r n^{\frac{1}{2}-\epsilon} \exp \left(-\frac{n^{2 \epsilon}}{8 \sigma^{2}}\right),
$$

which implies that $\lim _{n \rightarrow \infty} P_{\text {error }}^{(n)}=0$. Therefore, using (26), the rate

$$
R=\lim _{n \rightarrow \infty} \frac{\log \left(\left(\begin{array}{c}
k+r-1 \\
r-1
\end{array}\right)\right)}{\log (n)}=\frac{r-1}{2}-\epsilon(r-1)
$$

is achievable for every $\epsilon \in\left(0, \frac{1}{2}\right)$, and $C_{\text {perm }}\left(P_{Z \mid X}\right) \geq \frac{r-1}{2}$. This completes the proof.

We now make two pertinent remarks regarding Theorem 1. Firstly, the randomized encoder and element-wise thresholding decoder presented in the achievability proof constitute a computationally tractable coding scheme. Indeed, unlike the random coding argument in traditional channel coding, the element-wise thresholding decoder takes $O(n)$ (i.e., linear) time, because constructing $\hat{P}_{y_{1}^{n}}$ from $y_{1}^{n}$ requires $O(n)$ time and computing (30) requires $O(\sqrt{n})$ time. Therefore, communication via noisy permutation channels appears to not require the development of conceptually sophisticated coding schemes to theoretically achieve capacity. (Of course, other code constructions could be of utility based on alternative practical considerations.) Furthermore, our achievability proof also implies the existence of a good deterministic code using a simple application of the probabilistic method (see, e.g., [48, Lemma 2.2]).

Secondly, although we have presented Theorem 1 under an average probability of error criterion, our achievability proof establishes a lower bound on noisy permutation channel capacity under a maximal probability of error criterion as well; see, e.g., (35). More generally, the noisy permutation channel capacity of a DMC remains the same under a maximal probability of error criterion. This follows from a straightforward 
expurgation argument similar to [19, Theorem 18.3, Corollary 18.1] or [18, Section 7.7, p.204]. ${ }^{7}$

For the special case where $r=\operatorname{rank}\left(P_{Z \mid X}\right)=2$, we next present an alternative proof of Theorem 1 which exploits the second moment method bound in Lemma 5. For convenience, we also state the corresponding achievability result in the ensuing proposition.

Proposition 2 (Achievability Bound for DMCs with Rank 2). The noisy permutation channel capacity of a DMC $P_{Z \mid X}$ with $r \triangleq \operatorname{rank}\left(P_{Z \mid X}\right)=2$ is lower bounded by

$$
C_{\text {perm }}\left(P_{Z \mid X}\right) \geq \frac{1}{2} \text {. }
$$

Proof. We commence our proof without imposing the $r=2$ constraint. As in the proof of Theorem 1, consider the reduced input alphabet $\mathcal{X}^{\prime}=\{1, \ldots, r\} \subseteq \mathcal{X}$ such that the rows of $\tilde{P}_{Z \mid X}$ are linearly independent, the message set $\mathcal{M}=\mathcal{P}_{r, k}$ where $k=\left\lfloor n^{\frac{1}{2}-\epsilon}\right\rfloor$ for any $\epsilon \in\left(0, \frac{1}{2}\right)$, and the randomized encoder $f_{n}: \mathcal{P}_{r, k} \rightarrow \mathcal{X}^{n}$ given in (27) and (28). However, on the receiver end, consider the ML decoder $g_{n}: \mathcal{Y}^{n} \rightarrow \mathcal{P}_{r, k}$ such that

$$
\forall y_{1}^{n} \in \mathcal{Y}^{n}, g_{n}\left(y_{1}^{n}\right)=\underset{p \in \mathcal{P}_{r, k}}{\arg \max } P_{Y_{1}^{n} \mid M}\left(y_{1}^{n} \mid p\right),
$$

where the tie-breaking rule (to choose one maximizer when there are several) does not affect $P_{\text {error }}^{(n)}$. We now analyze the average probability of error for this simple encoding and decoding scheme.

Firstly, as before, we condition on the event $\{M=p\}$ for some $p=\left(\frac{p_{1}}{k}, \ldots, \frac{p_{r}}{k}\right) \in \mathcal{P}_{r, k}$, and note that

$$
X_{1}^{n} \stackrel{\text { i.i.d. }}{\sim} P_{X}, \quad Z_{1}^{n} \stackrel{\text { i.i.d. }}{\sim} P_{Z}, \quad Y_{1}^{n} \stackrel{\text { i.i.d. }}{\sim} P_{Z},
$$

where $P_{Z}$ denotes the output distribution when $P_{X}$, defined via (28), is "pushed forward" through $P_{Z \mid X}$. Moreover, as before, we let $\mathbb{P}_{p}$ represent the underlying probability measure after conditioning on $\{M=p\}$. The conditional probability that our ML decoder makes an error is upper bounded by

$$
\begin{aligned}
& \mathbb{P}_{p}(\hat{M} \neq M) \\
& =\mathbb{P}_{p}\left(g_{n}\left(Y_{1}^{n}\right) \neq p\right) \\
& \stackrel{\text { (a) }}{\leq} \mathbb{P}_{p}\left(\exists q \in \mathcal{P}_{r, k} \mid\{p\}, P_{Y_{1}^{n} \mid M}\left(Y_{1}^{n} \mid q\right) \geq P_{Y_{1}^{n} \mid M}\left(Y_{1}^{n} \mid p\right)\right) \\
& \stackrel{\text { (b) }}{\leq} \sum_{q \in \mathcal{P}_{r, k} \mid\{p\}} \mathbb{P}_{p}\left(P_{Y_{1}^{n} \mid M}\left(Y_{1}^{n} \mid q\right) \geq P_{Y_{1}^{n} \mid M}\left(Y_{1}^{n} \mid p\right)\right)
\end{aligned}
$$

where (a) is an upper bound because we regard the ML decoding equality case, $P_{Y_{1}^{n} \mid M}\left(Y_{1}^{n} \mid q\right)=P_{Y_{1}^{n} \mid M}\left(Y_{1}^{n} \mid p\right)$ for $q \neq p$, as an error even though the ML decoder may return the correct message in this scenario, and (b) follows from the union bound.

Then, to prove that this upper bound in (37) vanishes, for any message $q=\left(\frac{q_{1}}{k}, \ldots, \frac{q_{r}}{k}\right) \in \mathcal{P}_{r, k} \mid\{p\}$, consider a binary hypothesis test with likelihoods given by

$$
\text { Given } H=0: Y_{1}^{n} \stackrel{\text { i.i.d. }}{\sim} P_{Z},
$$

\footnotetext{
${ }^{7}$ Indeed, $C_{\text {perm }}$ under a maximal probability of error criterion is clearly upper bounded by $C_{\text {perm }}$ under an average probability of error criterion, and expurgating the code used to achieve $C_{\text {perm }}$ under an average probability of error criterion shows that this bound can be met with equality.
}

$$
\text { Given } H=1: Y_{1}^{n} \stackrel{\text { i.i.j. }}{\sim} Q_{Z},
$$

where the hypotheses $H=0$ and $H=1$ correspond to the messages $M=p$ and $M=q$, respectively, and $Q_{Z} \in \mathcal{P}_{\mathcal{Y}}$ is the output distribution when the input distribution $Q_{X} \in \mathcal{P}_{\mathcal{X}}$, defined analogously to (28) as

$$
Q_{X}(x)=\left\{\begin{array}{ll}
\frac{q_{x}}{k}, & \text { for } x \in \mathcal{X}^{\prime} \\
0, & \text { for } x \in \mathcal{X} \backslash \mathcal{X}^{\prime}
\end{array},\right.
$$

is "pushed forward" through the channel $P_{Z \mid X}$. Notice that the $\ell^{2}$-distance between $P_{Z}$ and $Q_{Z}$ can be upper and lower bounded using $\left\|P_{X}-Q_{X}\right\|_{2} ;{ }^{8}$ indeed,

$$
\begin{aligned}
\sigma_{\min }\left(\tilde{P}_{Z \mid X}\right)\left\|P_{X}-Q_{X}\right\|_{2} & \leq\left\|P_{Z}-Q_{Z}\right\|_{2} \\
& \leq\left\|P_{Z \mid X}\right\|_{\text {op }}\left\|P_{X}-Q_{X}\right\|_{2},
\end{aligned}
$$

where $\sigma_{\min }\left(\tilde{P}_{Z \mid X}\right)>0$ because the rows of $\tilde{P}_{Z \mid X}$ are linearly independent, the first inequality follows from the CourantFischer-Weyl min-max theorem, cf. [49, Theorem 7.3.8], because $\left\|P_{X}-Q_{X}\right\|_{2}=\|p-q\|_{2}$, and $P_{Z}$ and $Q_{Z}$ can be obtained by pushing $p$ and $q$ forward through the channel $\tilde{P}_{Z \mid X}$, respectively, and the second inequality follows from the definition of operator norm. So, letting

$$
\epsilon_{n}=\epsilon+\frac{\log \left(\sigma_{\min }\left(\tilde{P}_{Z \mid X}\right)\right)+\frac{1}{2} \log \left(\sum_{i=1}^{r}\left(p_{i}-q_{i}\right)^{2}\right)}{\log (n)}
$$

such that $\epsilon_{n} \in\left(0, \frac{1}{2}\right)$ for all sufficiently large $n$ (depending on $\left.P_{Z \mid X}\right)$, we have

$$
\begin{aligned}
\left\|P_{Z}-Q_{Z}\right\|_{2} & \geq \sigma_{\min }\left(\tilde{P}_{Z \mid X}\right)\|p-q\|_{2} \\
& =\frac{\sigma_{\min }\left(\tilde{P}_{Z \mid X}\right)}{\left\lfloor n^{\frac{1}{2}-\epsilon}\right\rfloor} \sqrt{\sum_{i=1}^{r}\left(p_{i}-q_{i}\right)^{2}} \\
& \geq \frac{1}{n^{\frac{1}{2}-\epsilon_{n}}},
\end{aligned}
$$

where $p=\left(\frac{p_{1}}{k}, \ldots, \frac{p_{r}}{k}\right) \in \mathcal{P}_{r, k}$ and $q=\left(\frac{q_{1}}{k}, \ldots, \frac{q_{r}}{k}\right) \in$ $\mathcal{P}_{r, k}$ with $k=\left\lfloor n^{\frac{1}{2}-\epsilon}\right\rfloor$. Using Lemma 5 (which is based on the second moment method in Lemma 4), if $H \sim \operatorname{Ber}\left(\frac{1}{2}\right)$, i.e., the hypotheses are equiprobable, then the ML decoding probability of error for our binary hypothesis testing problem, $P_{\mathrm{ML}}^{(n)}=\mathbb{P}\left(\hat{H}_{\mathrm{ML}}^{n}\left(Y_{1}^{n}\right) \neq H\right)$, satisfies

$$
\begin{aligned}
P_{\mathrm{ML}}^{(n)} & =\frac{1}{2} \mathbb{P}_{p}\left(\hat{H}_{\mathrm{ML}}^{n}\left(Y_{1}^{n}\right)=1\right)+\frac{1}{2} \mathbb{P}_{q}\left(\hat{H}_{\mathrm{ML}}^{n}\left(Y_{1}^{n}\right)=0\right) \\
& \leq \frac{|\mathcal{Y}|}{2|\mathcal{Y}|+2 n^{2 \epsilon_{n}}} .
\end{aligned}
$$

This implies that the false alarm probability satisfies

$$
\begin{aligned}
\mathbb{P}_{p}\left(\hat{H}_{\mathrm{ML}}^{n}\left(Y_{1}^{n}\right)=1\right) & =\mathbb{P}_{p}\left(P_{Y_{1}^{n} \mid M}\left(Y_{1}^{n} \mid q\right) \geq P_{Y_{1}^{n} \mid M}\left(Y_{1}^{n} \mid p\right)\right) \\
& \leq \frac{|\mathcal{Y}|}{|\mathcal{Y}|+n^{2 \epsilon_{n}}},
\end{aligned}
$$

where the equality follows from breaking ties in ML decoding, i.e., in cases where we get $P_{Y_{1}^{n} \mid M}\left(Y_{1}^{n} \mid q\right)=P_{Y_{1}^{n} \mid M}\left(Y_{1}^{n} \mid p\right)$, by

${ }^{8}$ The ensuing lower bound is where we crucially introduce a dependence between the noisy permutation channel capacity and rank of a DMC. Furthermore, the upper and lower bounds together imply that $\left\|P_{Z}-Q_{Z}\right\|_{2}=$ $\Theta\left(\left\|P_{X}-Q_{X}\right\|_{2}\right)$. 
assigning $\hat{H}_{\mathrm{ML}}^{n}\left(Y_{1}^{n}\right)=1$ (which does not affect the analysis of $P_{\mathrm{ML}}^{(n)}$ in Lemma 5).

Next, combining (37) and (40) yields

$$
\begin{aligned}
\mathbb{P}_{p}(\hat{M} \neq M) & \leq \sum_{q \in \mathcal{P}_{r, k} \mid\{p\}} \frac{|\mathcal{Y}|}{|\mathcal{Y}|+n^{2 \epsilon_{n}}} \\
& \leq|\mathcal{Y}| \sum_{q \in \mathcal{P}_{r, k} \mid\{p\}} \frac{1}{n^{2 \epsilon_{n}}} \\
& =\frac{|\mathcal{Y}|}{\sigma_{\min }\left(\tilde{P}_{Z \mid X}\right)^{2} n^{2 \epsilon}} \sum_{q \in \mathcal{P}_{r, k} \mid\{p\}} \frac{1}{\sum_{i=1}^{r}\left(p_{i}-q_{i}\right)^{2}},
\end{aligned}
$$

where the last equality follows from substituting (39). At this point, we use the fact that $r=2$ to simplify (41) so that

$$
\begin{aligned}
\mathbb{P}_{p}(\hat{M} \neq M) & \stackrel{(\text { a) }}{\leq} \frac{|\mathcal{Y}|}{2 \sigma_{\min }\left(\tilde{P}_{Z \mid X}\right)^{2} n^{2 \epsilon}} \sum_{q \in \mathcal{P}_{r, k} \backslash\{p\}} \frac{1}{\left(p_{1}-q_{1}\right)^{2}} \\
& \stackrel{\text { (b) }}{\leq} \frac{|\mathcal{Y}|}{\sigma_{\min }\left(\tilde{P}_{Z \mid X}\right)^{2} n^{2 \epsilon}} \sum_{j=1}^{\infty} \frac{1}{j^{2}} \\
& \stackrel{\text { (c) }}{=} \frac{|\mathcal{Y}| \pi^{2}}{6 \sigma_{\min }\left(\tilde{P}_{Z \mid X}\right)^{2} n^{2 \epsilon}}
\end{aligned}
$$

where (a) holds because $p_{1}+p_{2}=q_{1}+q_{2}=k$, (b) holds because $j=p_{1}-q_{1}$ ranges over a subset of all non-zero integers, and (c) utilizes the renowned solution to the Basel problem.

Finally, taking expectations with respect to the law of $M$ in (42) produces

$$
P_{\text {error }}^{(n)} \leq \frac{|\mathcal{Y}| \pi^{2}}{6 \sigma_{\min }\left(\tilde{P}_{Z \mid X}\right)^{2} n^{2 \epsilon}},
$$

which implies that $\lim _{n \rightarrow \infty} P_{\text {error }}^{(n)}=0$. Therefore, as argued in the proof of Theorem $1, C_{\text {perm }}\left(P_{Z \mid X}\right) \geq \frac{r-1}{2}=\frac{1}{2}$, which completes the proof.

In view of Proposition 2, some further remarks are in order. Firstly, the high-level proof strategy to establish Proposition 2 parallels the pairwise error probability analysis technique used in conventional channel coding problems (see, e.g., [50]). However, the details of our hypothesis testing formulation and the bounds we use to execute our analysis are different to such classical approaches.

Secondly, if $r>2$, then the obvious approach to bounding $P_{\text {error }}^{(n)}$ starting from (41) (and taking expectations with respect to $M$ ) yields

$$
P_{\text {error }}^{(n)} \leq \frac{|\mathcal{Y}|}{\sigma_{\min }\left(\tilde{P}_{Z \mid X}\right)^{2} n^{2 \epsilon}} \sum_{m \in \mathbb{Z}^{r} \backslash(0, \ldots, 0)} \frac{1}{\|m\|_{2}^{2}},
$$

because we can define $m=\left(m_{1}, \ldots, m_{r}\right) \in \mathbb{Z}^{r}$ such that $m_{i}=p_{i}-q_{i}$ for every $i \in\{1, \ldots, r\}$, and then take the summation over additional sequences $m$ whose sums are not necessarily equal to 0 (i.e., we take the summation over additional $q_{1}, \ldots, q_{r}$ whose sums are not necessarily equal to $k$, as is the case when $q \in \mathcal{P}_{r, k}$ ). It is straightforward to verify that the bound in (44) diverges when $r \geq 2$. Indeed, notice that

$$
\begin{aligned}
\sum_{m \in \mathbb{Z}^{r} \backslash(0, \ldots, 0)} \frac{1}{\|m\|_{2}^{2}} & \geq \sum_{m \in \mathbb{Z}^{r} \backslash(0, \ldots, 0)} \frac{1}{\|m\|_{1}^{2}} \\
& \geq \frac{1}{(r-1) !} \sum_{d=1}^{\infty} \frac{1}{d^{2}} \prod_{j=1}^{r-1}(d+j) \\
& \geq \frac{1}{(r-1) !} \sum_{d=1}^{\infty} \frac{1}{d^{3-r}}=+\infty,
\end{aligned}
$$

where the first inequality uses the monotonicity of $\ell^{p}$-norms in $p \in[1, \infty]$, the second inequality follows from enumerating over all possible $\ell^{1}$-norms $d$ and noting that there are $\left(\begin{array}{c}d+r-1 \\ r-1\end{array}\right)$ (entry-wise) non-negative points in the integer lattice $\mathbb{Z}^{r}$ that have an $\ell^{1}$-norm of $d$, and the expression in the final inequality is infinity due to the divergent nature of the harmonic series. In the $r=2$ case, as in the proof of Proposition 2 above, it is possible to tighten (44) and obtain a summation over $\mathbb{Z}$ rather than $\mathbb{Z}^{2}$. However, such a tightening does not ameliorate the divergent situation for $r>2$. So, the proof technique of Proposition 2 cannot be used for $r>2$.

Thirdly, as in the earlier proof of Theorem 1, the randomized encoder and ML decoder presented in the proof of Proposition 2 also constitute a computationally tractable coding scheme. In particular, the ML decoder requires at most $O(\sqrt{n})$ likelihood ratio tests, which means that the decoder operates in polynomial time in $n$.

Fourthly, for any non-trivial DMCs, we intuitively expect the rate of decay of $P_{\text {error }}^{(n)}$ to be dominated by the rate of decay of the probability of error in distinguishing between two "consecutive" messages. Although we do not derive precise error exponents or rates of decay in this paper, (35), (36), Lemma 5, and (43) indicate that this intuition is accurate.

Lastly, when we specialize (43) for a $\operatorname{BSC}(\delta)$ with $\delta \in$ $\left(0, \frac{1}{2}\right) \cup\left(\frac{1}{2}, 1\right)$, we get

$$
P_{\text {error }}^{(n)} \leq \frac{\pi^{2}}{3(1-2 \delta)^{2} n^{2 \epsilon}},
$$

because $|\mathcal{Y}|=|\{0,1\}|=2$ and $\sigma_{\min }\left(\tilde{P}_{Z \mid X}\right)=|1-2 \delta|$. This improves the constant in our corresponding bound in [2, Theorem 3, Equation (19)] by a factor of 3 .

\section{Converse Bounds for Strictly Positive DMCs}

In this subsection, we first prove Theorem 2. To this end, once again recall the formalism introduced in subsection I-B.

Proof of Theorem 2. Suppose we are given a sequence of encoder-decoder pairs $\left\{\left(f_{n}, g_{n}\right)\right\}_{n \in \mathbb{N}}$ on message sets of size $|\mathcal{M}|=n^{R}$ such that $\lim _{n \rightarrow \infty} P_{\text {error }}^{(n)}=0$. Consider the Markov chain $M \rightarrow X_{1}^{n} \rightarrow Z_{1}^{n} \rightarrow Y_{1}^{n} \rightarrow \hat{P}_{Y_{1}^{n}}$. Observe using (2) that for every $y_{1}^{n} \in \mathcal{Y}^{n}$ and $m \in \mathcal{M}$,

$$
P_{Y_{1}^{n} \mid M}\left(y_{1}^{n} \mid m\right)=\left(\begin{array}{c}
n \\
n \hat{P}_{y_{1}^{n}}^{n}
\end{array}\right)^{-1} \mathbb{P}\left(\hat{P}_{Z_{1}^{n}}=\hat{P}_{y_{1}^{n}} \mid M=m\right) .
$$

Since $P_{Y_{1}^{n} \mid M}\left(y_{1}^{n} \mid m\right)$ depends on $y_{1}^{n}$ through $\hat{P}_{y_{1}^{n}}$, the FisherNeyman factorization theorem implies that $\hat{P}_{Y_{1}^{n}}$ is a sufficient 
statistic of $Y_{1}^{n}$ for $M$ [51, Theorem 3.6]. Then, following the standard argument from [18, Section 7.9], we have

$$
\begin{aligned}
R \log (n) & \stackrel{(\mathrm{a})}{=} H(M) \\
& \stackrel{(\mathrm{b})}{=} H(M \mid \hat{M})+I(M ; \hat{M}) \\
& \stackrel{(\mathrm{c})}{\leq} 1+P_{\mathrm{error}}^{(n)} R \log (n)+I\left(M ; Y_{1}^{n}\right) \\
& \stackrel{(\mathrm{d})}{=} 1+P_{\text {error }}^{(n)} R \log (n)+I\left(M ; \hat{P}_{Y_{1}^{n}}\right) \\
& \stackrel{(\mathrm{e})}{\leq} 1+P_{\text {error }}^{(n)} R \log (n)+I\left(X_{1}^{n} ; \hat{P}_{Y_{1}^{n}}\right),
\end{aligned}
$$

where (a) holds because $M$ is uniformly distributed, (b) follows from the definition of mutual information, (c) follows from Fano's inequality and the data processing inequality [18, Theorems 2.10 .1 and 2.8.1], (d) holds because $\hat{P}_{Y_{1}^{n}}$ is a sufficient statistic, cf. [18, Section 2.9], and (e) also follows from the data processing inequality.

We now upper bound $I\left(X_{1}^{n} ; \hat{P}_{Y_{1}^{n}}\right)$. Notice that

$$
\begin{aligned}
I\left(X_{1}^{n} ; \hat{P}_{Y_{1}^{n}}\right)= & H\left(\hat{P}_{Y_{1}^{n}}\right)-H\left(\hat{P}_{Y_{1}^{n}} \mid X_{1}^{n}\right) \\
\leq & (|\mathcal{Y}|-1) \log (n+1) \\
& -\sum_{x_{1}^{n} \in\{0,1\}^{n}} P_{X_{1}^{n}}\left(x_{1}^{n}\right) H\left(\hat{P}_{Y_{1}^{n}} \mid X_{1}^{n}=x_{1}^{n}\right),
\end{aligned}
$$

where we use the upper bound on the number of possible empirical distributions given in (5). Given $X_{1}^{n}=x_{1}^{n}$ for any fixed $x_{1}^{n} \in \mathcal{X}^{n},\left\{Z_{i} \sim P_{Z \mid X}\left(\cdot \mid x_{i}\right): i \in\{1, \ldots, n\}\right\}$ are mutually independent and $\hat{P}_{Z_{1}^{n}}=\hat{P}_{Y_{1}^{n}}$ (almost surely). To lower bound $H\left(\hat{P}_{Y_{1}^{n}} \mid X_{1}^{n}=x_{1}^{n}\right)$, we need some additional definitions. Let $x^{*}=\arg \max _{x \in \mathcal{X}} \hat{P}_{x_{1}^{n}}(x)$, choosing one maximizer arbitrarily if there are several. For every $y \in \mathcal{Y}$, define the random variable

$$
N_{y} \triangleq \sum_{i=1}^{n} \mathbb{1}\left\{x_{i}=x^{*}\right\} \mathbb{1}\left\{Z_{i}=y\right\} .
$$

Furthermore, define the empirical conditional distribution

$$
\hat{P}_{Z \mid X=x}^{n}(y) \triangleq \frac{1}{n \hat{P}_{x_{1}^{n}}(x)} \sum_{i=1}^{n} \mathbb{1}\left\{x_{i}=x\right\} \mathbb{1}\left\{Z_{i}=y\right\}
$$

for every $y \in \mathcal{Y}$ and every $x \in \mathcal{X}$ such that $\hat{P}_{x_{1}^{n}}(x)>0$, where we let $\hat{P}_{Z \mid X=x}^{n}=\left(\hat{P}_{Z \mid X=x}^{n}(y): y \in \mathcal{Y}\right) \in \mathcal{P}_{\mathcal{Y}}$ so that

$$
\hat{P}_{Z_{1}^{n}}=\sum_{x \in \mathcal{X}} \hat{P}_{x_{1}^{n}}(x) \hat{P}_{Z \mid X=x}^{n} .
$$

In the sequel, for any $x \in \mathcal{X}$, if $\hat{P}_{x_{1}^{n}}(x)=0$, then we interpret $\hat{P}_{x_{1}^{n}}(x) \hat{P}_{Z \mid X=x}^{n}$ as the zero vector. Using these definitions, we have

$$
\begin{aligned}
& H\left(\hat{P}_{Y_{1}^{n}} \mid X_{1}^{n}=x_{1}^{n}\right) \\
& \stackrel{(\mathrm{a})}{=} H\left(\hat{P}_{Z_{1}^{n}} \mid X_{1}^{n}=x_{1}^{n}\right) \\
& \stackrel{\text { (b) }}{=} H\left(\sum_{x \in \mathcal{X}} \hat{P}_{x_{1}^{n}}(x) \hat{P}_{Z \mid X=x}^{n} \mid X_{1}^{n}=x_{1}^{n}\right) \\
& \stackrel{\text { (c) }}{\geq} H\left(\hat{P}_{x_{1}^{n}}\left(x^{*}\right) \hat{P}_{Z \mid X=x^{*}}^{n} \mid X_{1}^{n}=x_{1}^{n}\right)
\end{aligned}
$$

$\stackrel{(\mathrm{d})}{=} H\left(N_{1}, \ldots, N_{|\mathcal{Y}|-1} \mid X_{1}^{n}=x_{1}^{n}\right)$

$\stackrel{(\mathrm{e})}{=} H\left(N_{1} \mid X_{1}^{n}=x_{1}^{n}\right)+\sum_{i=2}^{|\mathcal{Y}|-1} H\left(N_{i} \mid N_{1}, \ldots, N_{i-1}, X_{1}^{n}=x_{1}^{n}\right)$

$\stackrel{\text { (f) }}{\geq} \sum_{i=1}^{|\mathcal{Y}|-1} H\left(N_{i} \mid\left\{N_{j}: j \in \mathcal{Y} \backslash\{i,|\mathcal{Y}|\}\right\}, X_{1}^{n}=x_{1}^{n}\right)$,

where (a) holds because $\hat{P}_{Z_{1}^{n}}=\hat{P}_{Y_{1}^{n}}$ (almost surely), (b) uses (49), (c) follows from [18, Problem 2.14] because $\left\{\hat{P}_{Z \mid X=x}^{n} \in\right.$ $\left.\mathcal{P}_{\mathcal{Y}}: x \in \mathcal{X}\right\}$ are mutually (conditionally) independent random variables given $X_{1}^{n}=x_{1}^{n}$, (d) holds because $\hat{P}_{Z \mid X=x^{*}}^{n}$ sums to unity and we let $\mathcal{Y}=\{1, \ldots,|\mathcal{Y}|\}$ without loss of generality, (e) follows from the chain rule for Shannon entropy (and the summation is 0 when $|\mathcal{Y}|=2$ ), and (f) holds because conditioning reduces Shannon entropy (and it equals $H\left(N_{1} \mid X_{1}^{n}=x_{1}^{n}\right)$ when $\left.|\mathcal{Y}|=2\right)$.

We next lower bound $H\left(N_{1} \mid N_{2}, \ldots, N_{|\mathcal{Y}|-1}, X_{1}^{n}=x_{1}^{n}\right)$; the other terms in the sum in (50) can be lower bounded similarly. Let $p \triangleq P_{Z \mid X}\left(1 \mid x^{*}\right) /\left(P_{Z \mid X}\left(1 \mid x^{*}\right)+P_{Z \mid X}\left(|\mathcal{Y}| \mid x^{*}\right)\right)$. Then, the conditional distribution of $N_{1}$ given $N_{2}, \ldots, N_{|\mathcal{Y}|-1}$ and $X_{1}^{n}=x_{1}^{n}$ is given by

$$
\begin{aligned}
& \mathbb{P}\left(N_{1}=k_{1} \mid N_{2}=k_{2}, \ldots, N_{|\mathcal{Y}|-1}=k_{|\mathcal{Y}|-1}, X_{1}^{n}=x_{1}^{n}\right) \\
& =\frac{\mathbb{P}\left(N_{1}=k_{1}, \ldots, N_{|\mathcal{Y}|-1}=k_{|\mathcal{Y}|-1} \mid X_{1}^{n}=x_{1}^{n}\right)}{\mathbb{P}\left(N_{2}=k_{2}, \ldots, N_{|\mathcal{Y}|-1}=k_{|\mathcal{Y}|-1} \mid X_{1}^{n}=x_{1}^{n}\right)} \\
& \prod_{j \in \mathcal{Y}} \frac{P_{Z \mid X}\left(j \mid x^{*}\right)^{k_{j}}}{k_{j} !} \\
& \frac{\overline{\left(P_{Z \mid X}\left(1 \mid x^{*}\right)+P_{Z \mid X}\left(|\mathcal{Y}| \mid x^{*}\right)\right)^{k_{1}+k_{|\mathcal{Y}|}}}}{\left(k_{1}+k_{|\mathcal{Y}|}\right) !} \prod_{j=2}^{|\mathcal{Y}|-1} \frac{P_{Z \mid X}\left(j \mid x^{*}\right)^{k_{j}}}{k_{j} !} \\
& =\left(\begin{array}{c}
k_{1}+k_{|\mathcal{Y}|} \\
k_{1}
\end{array}\right) p^{k_{1}}(1-p)^{k_{|\mathcal{Y}|}}
\end{aligned}
$$

for every $k_{1}, \ldots, k_{|\mathcal{Y}|} \in \mathbb{N} \cup\{0\}$ such that $\sum_{j \in \mathcal{Y}} k_{j}=$ $n \hat{P}_{x_{1}^{n}}\left(x^{*}\right)$. Therefore, $N_{1} \sim \operatorname{bin}\left(n \hat{P}_{x_{1}^{n}}\left(x^{*}\right)-\sum_{j=2}^{|\mathcal{Y}|-1} k_{j}, p\right)$ given $N_{2}=k_{2}, \ldots, N_{|\mathcal{Y}|-1}=k_{|\mathcal{Y}|-1}$ and $X_{1}^{n}=x_{1}^{n}$. (We remark that this calculation also holds for the $|\mathcal{Y}|=2$ case because $\sum_{j=2}^{|\mathcal{Y}|-1} k_{j}=0$.) Now, for some fixed constant $\tau \in\left(1-P_{Z \mid X}\left(1 \mid x^{*}\right)-P_{Z \mid X}\left(|\mathcal{Y}| \mid x^{*}\right), 1\right)$, let $E \in\{0,1\}$ be a binary random variable defined by

$$
E \triangleq \mathbb{1}\left\{\sum_{j=2}^{|\mathcal{Y}|-1} N_{j} \leq n \hat{P}_{x_{1}^{n}}\left(x^{*}\right) \tau\right\}
$$

Observe using the Bienaymé-Chebyshev inequality that

$$
\begin{aligned}
& \mathbb{P}\left(E=0 \mid X_{1}^{n}=x_{1}^{n}\right) \\
& =\mathbb{P}\left(\hat{P}_{Z \mid X=x^{*}}^{n}(1)+\hat{P}_{Z \mid X=x^{*}}^{n}(|\mathcal{Y}|)<1-\tau \mid X_{1}^{n}=x_{1}^{n}\right) \\
& \stackrel{\text { (a) }}{\leq} \frac{\mathbb{V} \mathbb{R}\left(\hat{P}_{Z \mid X=x^{*}}^{n}(1)+\hat{P}_{Z \mid X=x^{*}}^{n}(|\mathcal{Y}|) \mid X_{1}^{n}=x_{1}^{n}\right)}{\left(\tau+P_{Z \mid X}\left(\{1,|\mathcal{Y}|\} \mid x^{*}\right)-1\right)^{2}} \\
& \stackrel{\text { (b) }}{=} \frac{P_{Z \mid X}\left(\{1,|\mathcal{Y}|\} \mid x^{*}\right)\left(1-P_{Z \mid X}\left(\{1,|\mathcal{Y}|\} \mid x^{*}\right)\right)}{n \hat{P}_{x_{1}^{n}}\left(x^{*}\right)\left(\tau+P_{Z \mid X}\left(\{1,|\mathcal{Y}|\} \mid x^{*}\right)-1\right)^{2}} \\
& \stackrel{\text { (c) }}{\leq} \frac{|\mathcal{X}| P_{Z \mid X}\left(\{1,|\mathcal{Y}|\} \mid x^{*}\right)\left(1-P_{Z \mid X}\left(\{1,|\mathcal{Y}|\} \mid x^{*}\right)\right)}{n\left(\tau+P_{Z \mid X}\left(\{1,|\mathcal{Y}|\} \mid x^{*}\right)-1\right)^{2}}
\end{aligned}
$$




$$
=O\left(\frac{1}{n}\right)
$$

where (a), (b), and (c) use the notation $P_{Z \mid X}\left(\{1,|\mathcal{Y}|\} \mid x^{*}\right)=$ $P_{Z \mid X}\left(1 \mid x^{*}\right)+P_{Z \mid X}\left(|\mathcal{Y}| \mid x^{*}\right)$, and (c) also utilizes the fact that $\hat{P}_{x_{1}^{n}}\left(x^{*}\right) \geq \frac{1}{|\mathcal{X}|}$ by definition of $x^{*}$. Then, we can apply Lemma 1 and get

$$
\begin{aligned}
& H\left(N_{1} \mid N_{2}, \ldots, N_{|\mathcal{Y}|-1}, X_{1}^{n}=x_{1}^{n}\right) \\
& \stackrel{\text { (a) }}{\geq} H\left(N_{1} \mid N_{2}, \ldots, N_{|\mathcal{Y}|-1}, E, X_{1}^{n}=x_{1}^{n}\right) \\
& \stackrel{\text { (b) }}{\geq}\left(1-O\left(\frac{1}{n}\right)\right) H\left(N_{1} \mid N_{2}, \ldots, N_{|\mathcal{Y}|-1}, E=1, X_{1}^{n}=x_{1}^{n}\right) \\
& =\left(1-O\left(\frac{1}{n}\right)\right) . \\
& \quad \mathbb{E}\left[H\left(\operatorname{bin}\left(n \hat{P}_{x_{1}^{n}}\left(x^{*}\right)-\sum_{j=2}^{|\mathcal{Y}|-1} N_{j}, p\right)\right) \mid E=1, X_{1}^{n}=x_{1}^{n}\right] \\
& \left.\stackrel{\text { (c) }}{\geq}\left(1-O\left(\frac{1}{n}\right)\right) H\left(\operatorname{bin}\left(\mid \frac{n(1-\tau)}{|\mathcal{X}|}\right], p\right)\right) \\
& \stackrel{\text { (d) }}{\geq}\left(1-O\left(\frac{1}{n}\right)\right) \frac{1}{2} \log \left(2 \pi e p(1-p)\left(\frac{n(1-\tau)}{|\mathcal{X}|}-1\right)\right) \\
& \quad-\left(1-O\left(\frac{1}{n}\right)\right) \frac{c(p)|\mathcal{X}|}{n(1-\tau)-|\mathcal{X}|},
\end{aligned}
$$

where (a) holds because conditioning reduces Shannon entropy, (b) follows from (52) and the non-negativity of Shannon entropy, (c) follows from [18, Problem 2.14] and the facts that $E=1$ and $\hat{P}_{x_{1}^{n}}\left(x^{*}\right) \geq \frac{1}{|\mathcal{X}|}$, and (d) employs Lemma 1. Here, to employ Lemma 1 and obtain (53), we implicitly utilize the assumption that all conditional distributions in $\left\{P_{Z \mid X}(\cdot \mid x) \in \mathcal{P}_{\mathcal{Y}}: x \in \mathcal{X}\right\}$ are strictly positive, which ensures that $p \in(0,1) .{ }^{9}$ We also note that when $|\mathcal{Y}|=2$, the above argument mutatis mutandis yields

$$
\begin{aligned}
H\left(N_{1} \mid\right. & \left.X_{1}^{n}=x_{1}^{n}\right) \\
& =H\left(\operatorname{bin}\left(n \hat{P}_{x_{1}^{n}}\left(x^{*}\right), p\right)\right) \\
\geq & \frac{1}{2} \log \left(2 \pi e p(1-p)\left(\frac{n}{|\mathcal{X}|}-1\right)\right)-\frac{c(p)|\mathcal{X}|}{n-|\mathcal{X}|},
\end{aligned}
$$

which is lower bounded by (53). So, the bound in (53) is valid for all $|\mathcal{Y}| \geq 2$.

Next, to upper bound $I\left(X_{1}^{n} ; \hat{P}_{Y_{1}^{n}}\right)$, let $\tau^{*}$ be any fixed constant such that

$$
1-\min _{\substack{x \in \mathcal{X}, y, y^{\prime} \in \mathcal{Y}: \\ y \neq y^{\prime}}} P_{Z \mid X}\left(\left\{y, y^{\prime}\right\} \mid x\right)<\tau^{*}<1
$$

where $P_{Z \mid X}\left(\left\{y, y^{\prime}\right\} \mid x\right)=P_{Z \mid X}(y \mid x)+P_{Z \mid X}\left(y^{\prime} \mid x\right)$ for any $x \in \mathcal{X}$ and $y, y^{\prime} \in \mathcal{Y}$ such that $y \neq y^{\prime}$. Notice that when we analyze other conditional entropy terms $H\left(N_{i} \mid\left\{N_{j}: j \in\right.\right.$ $\left.\mathcal{Y} \backslash\{i,|\mathcal{Y}|\}\}, X_{1}^{n}=x_{1}^{n}\right)$ for $i \in\{1, \ldots,|\mathcal{Y}|-1\}$ akin to our

\footnotetext{
${ }^{9}$ Note that since we do not know a priori which value $x^{*}$ takes and we have to prove (53) for every term in (50), we have to assume that $P_{Z \mid X}(y \mid x)>0$ for all $x \in \mathcal{X}$ and $y \in \mathcal{Y}$.
}

analysis of $H\left(N_{1} \mid N_{2}, \ldots, N_{|\mathcal{Y}|-1}, X_{1}^{n}=x_{1}^{n}\right)$ above with $\tau=$ $\tau^{*}$, the maximum bound of the form (51) is

$$
\max _{\substack{x \in \mathcal{X} \\ y, y^{\prime} \in \mathcal{Y} \\ y \neq y^{\prime}}} \frac{|\mathcal{X}| P_{Z \mid X}\left(\left\{y, y^{\prime}\right\} \mid x\right)\left(1-P_{Z \mid X}\left(\left\{y, y^{\prime}\right\} \mid x\right)\right)}{n\left(\tau^{*}+P_{Z \mid X}\left(\left\{y, y^{\prime}\right\} \mid x\right)-1\right)^{2}}=O\left(\frac{1}{n}\right)
$$

which remains $O\left(\frac{1}{n}\right)$. Furthermore, let $p^{*}$ be the optimal value of

$$
p=\frac{P_{Z \mid X}(y \mid x)}{P_{Z \mid X}\left(\left\{y, y^{\prime}\right\} \mid x\right)}>0
$$

that minimizes (53), with $\tau=\tau^{*}$ and the $O\left(\frac{1}{n}\right)$ term given by (54), over all $x \in \mathcal{X}$ and $y, y^{\prime} \in \mathcal{Y}$ such that $y \neq y^{\prime}$. Then, following the derivation of (53), for all $i \in\{1, \ldots,|\mathcal{Y}|-1\}$, we obtain the lower bound

$$
\begin{aligned}
& H\left(N_{i} \mid\left\{N_{j}: j \in \mathcal{Y} \backslash\{i,|\mathcal{Y}|\}\right\}, X_{1}^{n}=x_{1}^{n}\right) \\
& \geq\left(1-O\left(\frac{1}{n}\right)\right) \frac{1}{2} \log \left(2 \pi e p^{*}\left(1-p^{*}\right)\left(\frac{n\left(1-\tau^{*}\right)}{|\mathcal{X}|}-1\right)\right) \\
& \quad-\left(1-O\left(\frac{1}{n}\right)\right) \frac{c\left(p^{*}\right)|\mathcal{X}|}{n\left(1-\tau^{*}\right)-|\mathcal{X}|},
\end{aligned}
$$

where the $O\left(\frac{1}{n}\right)$ term is given by (54). Hence, we can combine (48), (50), and (55) to produce

$$
\begin{aligned}
& I\left(X_{1}^{n} ; \hat{P}_{Y_{1}^{n}}\right) \\
& \begin{aligned}
\leq(|\mathcal{Y}|-1)( & \log (n+1)-\left(1-O\left(\frac{1}{n}\right)\right) . \\
& \left(\frac{1}{2} \log \left(2 \pi e p^{*}\left(1-p^{*}\right)\left(\frac{n\left(1-\tau^{*}\right)}{|\mathcal{X}|}-1\right)\right)\right. \\
& \left.\left.-\frac{c\left(p^{*}\right)|\mathcal{X}|}{n\left(1-\tau^{*}\right)-|\mathcal{X}|}\right)\right) .
\end{aligned}
\end{aligned}
$$

Finally, combining (47) and (56), and dividing by $\log (n)$, yields

$$
\begin{aligned}
R \leq \frac{1}{\log (n)}+P_{\text {error }}^{(n)} R+(|\mathcal{Y}|-1)\left(\frac{\log (n+1)}{\log (n)}-\right. & \\
\left(1-O\left(\frac{1}{n}\right)\right)\left(\frac{\log \left(2 \pi e p^{*}\left(1-p^{*}\right)\left(1-\tau^{*}\right) /|\mathcal{X}|\right)}{2 \log (n)}+\right. & \frac{\log \left(n-\left(|\mathcal{X}| /\left(1-\tau^{*}\right)\right)\right)}{2 \log (n)}- \\
& \left.\left.\frac{c\left(p^{*}\right)|\mathcal{X}|}{\left(n\left(1-\tau^{*}\right)-|\mathcal{X}|\right) \log (n)}\right)\right)
\end{aligned}
$$

where letting $n \rightarrow \infty$ produces

$$
R \leq \frac{|\mathcal{Y}|-1}{2}
$$

This completes the proof.

The proofs of Lemmata 4 and 5 in appendices B and C (along with the discussion following Lemmata 4 and 5) and the proof of Proposition 2 portray that the distinguishability between two "consecutive" (encoded) messages can be determined by a careful comparison of the difference between their means and a variance (at least in the rank 2 case). This suggests that the CLT can be used to obtain the correct scaling 
of $|\mathcal{M}|$ with $n$ in general. We remark that the CLT is in fact implicitly used in the above converse proof when we apply Lemma 1, because estimates for the entropy of a binomial distribution are typically obtained using the CLT.

We conclude this section by using Theorem 2, Proposition 1 , and Lemma 2 to establish the alternative converse bound on the noisy permutation channel capacity of strictly positive DMCs given in Theorem 3.

Proof of Theorem 3. As in the proof of Theorem 2, consider any sequence of encoder-decoder pairs $\left\{\left(f_{n}, g_{n}\right)\right\}_{n \in \mathbb{N}}$ on message sets of size $|\mathcal{M}|=n^{R}$ such that $\lim _{n \rightarrow \infty} P_{\text {error }}^{(n)}=0$. This defines the Markov chain $M \rightarrow X_{1}^{n} \rightarrow Z_{1}^{n} \rightarrow Y_{1}^{n}$, and the standard argument from [18, Section 7.9], which yielded (47) earlier, easily produces

$$
R \log (n) \leq 1+P_{\text {error }}^{(n)} R \log (n)+I\left(X_{1}^{n} ; Y_{1}^{n}\right) .
$$

We proceed to upper bounding $I\left(X_{1}^{n} ; Y_{1}^{n}\right)$ using a degradation argument.

First, we reduce the cardinality of the input alphabet $\mathcal{X}$ of the DMC $P_{Z \mid X}$. In particular, we let $\mathcal{X}^{\prime} \subseteq \mathcal{X}$ be any (fixed) subset of $\mathcal{X}$ such that $q \triangleq\left|\mathcal{X}^{\prime}\right|=\operatorname{ext}\left(P_{Z \mid X}\right)$ and the set of conditional distributions $\left\{P_{Z \mid X}(\cdot \mid x) \in \mathcal{P}_{\mathcal{Y}}: x \in \mathcal{X}^{\prime}\right\}$ (as vectors in $\left.\mathbb{R}^{|\mathcal{Y}|}\right)$ are the extreme points of the convex hull of $\left\{P_{Z \mid X}(\cdot \mid x) \in \mathcal{P}_{\mathcal{Y}}: x \in \mathcal{X}\right\} .{ }^{10}$ Moreover, we let $P_{Z \mid \tilde{X}} \in$ $\mathbb{R}^{q \times|\mathcal{Y}|}$ denote the row stochastic matrix whose rows are given by $\left\{P_{Z \mid X}(\cdot \mid x) \in \mathcal{P}_{\mathcal{Y}}: x \in \mathcal{X}^{\prime}\right\}$, where the random variable $\tilde{X} \in \mathcal{X}^{\prime}$. Since the convex hulls of $\left\{P_{Z \mid X}(\cdot \mid x) \in \mathcal{P}_{\mathcal{Y}}: x \in\right.$ $\left.\mathcal{X}^{\prime}\right\}$ and $\left\{P_{Z \mid X}(\cdot \mid x) \in \mathcal{P}_{\mathcal{Y}}: x \in \mathcal{X}\right\}$ are equivalent, for every $x \in \mathcal{X}$, we have

$$
\forall z \in \mathcal{Y}, \quad P_{Z \mid X}(z \mid x)=\sum_{\tilde{x} \in \mathcal{X}^{\prime}} Q_{x}(\tilde{x}) P_{Z \mid X}(z \mid \tilde{x})
$$

for some convex weights $\left\{Q_{x}(\tilde{x}) \geq 0: \tilde{x} \in \mathcal{X}^{\prime}\right\}$ such that $\sum_{\tilde{x} \in \mathcal{X}^{\prime}} Q_{x}(\tilde{x})=1$. Observe that for every probability distribution $P_{X_{1}^{n}} \in \mathcal{P}_{\mathcal{X}^{n}}$, we can construct the probability distribution $P_{\tilde{X}_{1}^{n}} \in \mathcal{P}\left(\mathcal{X}^{\prime}\right)^{n}$ given by

$$
\forall \tilde{x}_{1}^{n} \in\left(\mathcal{X}^{\prime}\right)^{n}, P_{\tilde{X}_{1}^{n}}\left(\tilde{x}_{1}^{n}\right) \triangleq \sum_{x_{1}^{n} \in \mathcal{X}^{n}} P_{X_{1}^{n}}\left(x_{1}^{n}\right) \prod_{i=1}^{n} Q_{x_{i}}\left(\tilde{x}_{i}\right),
$$

where the random variables $\tilde{X}_{1}, \ldots, \tilde{X}_{n} \in \mathcal{X}^{\prime}$. This distribution has the property that it induces the marginal distribution $P_{Z_{1}^{n}}$ of $Z_{1}^{n}$, namely, for all $z_{1}^{n} \in \mathcal{Y}^{n}$,

$$
\begin{aligned}
P_{Z_{1}^{n}}\left(z_{1}^{n}\right) & \triangleq \sum_{x_{1}^{n} \in \mathcal{X}^{n}} P_{X_{1}^{n}}\left(x_{1}^{n}\right) \prod_{i=1}^{n} P_{Z \mid X}\left(z_{i} \mid x_{i}\right) \\
& =\sum_{x_{1}^{n} \in \mathcal{X}^{n}} P_{X_{1}^{n}}\left(x_{1}^{n}\right) \prod_{i=1}^{n} \sum_{\tilde{x} \in \mathcal{X}^{\prime}} Q_{x_{i}}(\tilde{x}) P_{Z \mid X}\left(z_{i} \mid \tilde{x}\right) \\
& =\sum_{x_{1}^{n} \in \mathcal{X}^{n}} P_{X_{1}^{n}}\left(x_{1}^{n}\right) \sum_{\tilde{x}_{1}^{n} \in\left(\mathcal{X}^{\prime}\right)^{n}} \prod_{i=1}^{n} Q_{x_{i}}\left(\tilde{x}_{i}\right) P_{Z \mid X}\left(z_{i} \mid \tilde{x}_{i}\right)
\end{aligned}
$$

\footnotetext{
${ }^{10}$ We note that when there are multiple copies of an extreme point of the convex hull of $\left\{P_{Z \mid X}(\cdot \mid x) \in \mathcal{P}_{\mathcal{Y}}: x \in \mathcal{X}\right\}$ in $\left\{P_{Z \mid X}(\cdot \mid x) \in \mathcal{P}_{\mathcal{Y}}: x \in\right.$ $\mathcal{X}\}$, we only add one of these conditional distributions to $\left\{P_{Z \mid X}(\cdot \mid x) \in\right.$ $\left.\mathcal{P}_{\mathcal{Y}}: x \in \mathcal{X}^{\prime}\right\}$.
}

$$
\begin{aligned}
& =\sum_{\tilde{x}_{1}^{n} \in\left(\mathcal{X}^{\prime}\right)^{n}} \sum_{x_{1}^{n} \in \mathcal{X}^{n}} P_{X_{1}^{n}}\left(x_{1}^{n}\right) \prod_{i=1}^{n} Q_{x_{i}}\left(\tilde{x}_{i}\right) P_{Z \mid X}\left(z_{i} \mid \tilde{x}_{i}\right) \\
& =\sum_{\tilde{x}_{1}^{n} \in\left(\mathcal{X}^{\prime}\right)^{n}} P_{\tilde{X}_{1}^{n}}\left(\tilde{x}_{1}^{n}\right) \prod_{i=1}^{n} P_{Z \mid X}\left(z_{i} \mid \tilde{x}_{i}\right)
\end{aligned}
$$

where the first equality defines the distribution $P_{Z_{1}^{n}}$ of $Z_{1}^{n}$ (and uses the memorylessness of $P_{Z \mid X}$ ), the second equality follows from (58), the third equality follows from the distributive property, the fourth equality follows from swapping the order of summations, and the fifth equality follows from (59). Furthermore, notice that

$$
\begin{aligned}
& I\left(X_{1}^{n} ; Y_{1}^{n}\right) \\
& =\sum_{x_{1}^{n} \in \mathcal{X}^{n}} P_{X_{1}^{n}}\left(x_{1}^{n}\right) D\left(P_{Y_{1}^{n} \mid X_{1}^{n}}\left(\cdot \mid x_{1}^{n}\right)|| P_{Y_{1}^{n}}\right) \\
& \stackrel{(a)}{=} \sum_{x_{1}^{n} \in \mathcal{X}^{n}} P_{X_{1}^{n}}\left(x_{1}^{n}\right) D\left(P_{Z_{1}^{n} \mid X_{1}^{n}}\left(\cdot \mid x_{1}^{n}\right) \cdot \Pi|| P_{Y_{1}^{n}}\right) \\
& \stackrel{(\mathrm{b})}{=} \sum_{x_{1}^{n} \in \mathcal{X}^{n}} P_{X_{1}^{n}}\left(x_{1}^{n}\right) \text {. } \\
& D\left(\sum_{\tilde{x}_{1}^{n} \in\left(\mathcal{X}^{\prime}\right)^{n}} \prod_{i=1}^{n} Q_{x_{i}}\left(\tilde{x}_{i}\right)\left(P_{Z_{1}^{n} \mid X_{1}^{n}}\left(\cdot \mid \tilde{x}_{1}^{n}\right) \cdot \Pi\right) \| P_{Y_{1}^{n}}\right) \\
& \stackrel{(\mathrm{c})}{=} \sum_{x_{1}^{n} \in \mathcal{X}^{n}} P_{X_{1}^{n}}\left(x_{1}^{n}\right) \text {. } \\
& D\left(\sum_{\tilde{x}_{1}^{n} \in\left(\mathcal{X}^{\prime}\right)^{n}} \prod_{i=1}^{n} Q_{x_{i}}\left(\tilde{x}_{i}\right) P_{Y_{1}^{n} \mid X_{1}^{n}}\left(\cdot \mid \tilde{x}_{1}^{n}\right) \| P_{Y_{1}^{n}}\right) \\
& \stackrel{(\mathrm{d})}{\leq} \sum_{x_{1}^{n} \in \mathcal{X}^{n}} P_{X_{1}^{n}}\left(x_{1}^{n}\right) \sum_{\tilde{x}_{1}^{n} \in\left(\mathcal{X}^{\prime}\right)^{n}} \prod_{i=1}^{n} Q_{x_{i}}\left(\tilde{x}_{i}\right) D\left(P_{Y_{1}^{n} \mid X_{1}^{n}}\left(\cdot \mid \tilde{x}_{1}^{n}\right)|| P_{Y_{1}^{n}}\right) \\
& \stackrel{(\mathrm{e})}{=} \sum_{\tilde{x}_{1}^{n} \in\left(\mathcal{X}^{\prime}\right)^{n}} D\left(P_{Y_{1}^{n} \mid X_{1}^{n}}\left(\cdot \mid \tilde{x}_{1}^{n}\right)|| P_{Y_{1}^{n}}\right) \sum_{x_{1}^{n} \in \mathcal{X}^{n}} P_{X_{1}^{n}}\left(x_{1}^{n}\right) \prod_{i=1}^{n} Q_{x_{i}}\left(\tilde{x}_{i}\right) \\
& \stackrel{(\mathrm{f})}{=} \sum_{\tilde{x}_{1}^{n} \in\left(\mathcal{X}^{\prime}\right)^{n}} P_{\tilde{X}_{1}^{n}}\left(\tilde{x}_{1}^{n}\right) D\left(P_{Y_{1}^{n} \mid X_{1}^{n}}\left(\cdot \mid \tilde{x}_{1}^{n}\right)|| P_{Y_{1}^{n}}\right) \\
& \stackrel{(\mathrm{g})}{=} I\left(\tilde{X}_{1}^{n} ; Y_{1}^{n}\right) \text {, }
\end{aligned}
$$

where (a) and (c) hold because the conditional distribution $P_{Y_{1}^{n} \mid X_{1}^{n}}\left(\cdot \mid x_{1}^{n}\right)=P_{Z_{1}^{n} \mid X_{1}^{n}}\left(\cdot \mid x_{1}^{n}\right) \cdot \Pi \in \mathcal{P}_{\mathcal{Y}^{n}}$ is the output of pushing the conditional distribution $P_{Z_{1}^{n} \mid X_{1}^{n}}\left(\cdot \mid x_{1}^{n}\right) \in \mathcal{P}_{\mathcal{Y}^{n}}$ through the random permutation channel $\Pi=P_{Y_{1}^{n} \mid Z_{1}^{n}}$ (defined in (2)) for every $x_{1}^{n} \in \mathcal{X}^{n}$, (b) follows from (60) after substituting Kronecker delta distributions $P_{X_{1}^{n}}$ into (59) (and uses the memorylessness of $P_{Z \mid X}$ ), (d) follows from the convexity of KL divergence, (e) follows from swapping the order of summations, (f) follows from (59), and (g) holds because (60) conveys that $P_{Y_{1}^{n}} \in \mathcal{P}_{y^{n}}$, which is the marginal distribution of $Y_{1}^{n}$ in the original Markov chain $X_{1}^{n} \rightarrow Z_{1}^{n} \rightarrow Y_{1}^{n}$, is also the marginal distribution of $Y_{1}^{n}$ in the Markov chain $\tilde{X}_{1}^{n} \rightarrow Z_{1}^{n} \rightarrow Y_{1}^{n} \cdot{ }^{11}$

\footnotetext{
${ }^{11}$ Note that we abuse notation here and use the same random variable labels $Z_{1}^{n}$ and $Y_{1}^{n}$ for the Markov chains $X_{1}^{n} \rightarrow Z_{1}^{n} \rightarrow Y_{1}^{n}$ and $\tilde{X}_{1}^{n} \rightarrow Z_{1}^{n} \rightarrow$ $Y_{1}^{n}$, because the two chains can be coupled so that $Z_{1}^{n}$ and $Y_{1}^{n}$ are shared random variables.
} 
Second, we construct an equivalent model of the Markov chain $\tilde{X}_{1}^{n} \rightarrow Z_{1}^{n} \rightarrow Y_{1}^{n}$, which has reduced input alphabet $\mathcal{X}^{\prime}, \tilde{X}_{1}^{n} \sim P_{\tilde{X}_{1}^{n}}, P_{Z^{n} \mid \tilde{X}^{n}}$ given by the DMC $P_{Z \mid \tilde{X}}$, and $P_{Y_{1}^{n} \mid Z_{1}^{n}}=\Pi$ given by the random permutation channel in (2). Employing Lemma 2 (also see Figure 3), we can swap the random permutation channel $\Pi$ and the DMC $P_{Z \mid \tilde{X}}$ to get a Markov chain $\tilde{X}_{1}^{n} \rightarrow V_{1}^{n} \rightarrow W_{1}^{n}$ such that the channel $P_{W_{1}^{n} \mid \tilde{X}_{1}^{n}}$ is equivalent to the channel $P_{Y_{1}^{n} \mid \tilde{X}_{1}^{n}}$. In this alternative Markov chain, $V_{1}^{n} \in\left(\mathcal{X}^{\prime}\right)^{n}$ is an independent random permutation of $\tilde{X}_{1}^{n} \in\left(\mathcal{X}^{\prime}\right)^{n}$, and $W_{1}^{n} \in \mathcal{Y}^{n}$ is the output of passing $V_{1}^{n}$ through a DMC $P_{W \mid V}$, which satisfies $P_{W \mid V}=P_{Z \mid \tilde{X}}$ (as shown in (14)). Hence, we have

$$
I\left(\tilde{X}_{1}^{n} ; Y_{1}^{n}\right)=I\left(\tilde{X}_{1}^{n} ; W_{1}^{n}\right),
$$

since $\tilde{X}_{1}^{n}$ is common to both Markov chains $\tilde{X}_{1}^{n} \rightarrow Z_{1}^{n} \rightarrow Y_{1}^{n}$ and $\tilde{X}_{1}^{n} \rightarrow V_{1}^{n} \rightarrow W_{1}^{n}$.

Third, we construct a $q$-ary symmetric channel that dominates the DMC $P_{W \mid V}=P_{Z \mid \tilde{X}}$ in the degradation sense. To this end, define the parameter

$$
\delta=\frac{\nu}{1-\nu+\frac{\nu}{q-1}}>0
$$

in terms of the minimum entry, $\nu$, of $P_{Z \mid X}$, viz.,

$$
\nu=\min _{x \in \mathcal{X}, y \in \mathcal{Y}} P_{Z \mid X}(y \mid x)=\min _{x \in \mathcal{X}^{\prime}, y \in \mathcal{Y}} P_{Z \mid \tilde{X}}(y \mid x)>0,
$$

where the second equality follows from (58). (Note that $\delta>0$ because $\nu>0$, and $\nu>0$ since $P_{Z \mid X}$ is strictly positive.) Then, applying Proposition 1, we get that $P_{W \mid V}=P_{Z \mid \tilde{X}}$ is a degraded version of $q-\mathrm{SC}(\delta)$ (see Definition 3). Let the input random variable of the $q$-SC $(\delta)$ be $V \in \mathcal{X}^{\prime}$, and the output random random variable be $\tilde{W} \in \mathcal{X}^{\prime}$, so that we can write $P_{\tilde{W} \mid V}=S_{\delta}$. Now consider the Markov chain $\tilde{X}_{1}^{n} \rightarrow V_{1}^{n} \rightarrow \tilde{W}_{1}^{n}$, where $V_{1}^{n}$ is a random permutation of $\tilde{X}_{1}^{n}$ as before, and the channel $P_{\tilde{W}_{1}^{n} \mid V_{1}^{n}}$ is given by the DMC $P_{\tilde{W} \mid V}=S_{\delta}$. Since the degradation preorder tensorizes, the channel $P_{W \mid V}^{\otimes n}$ is a degraded version of the channel $P_{\tilde{W} \mid V}^{\otimes n}=S_{\delta}^{\otimes n}$, where $A^{\otimes n}$ denotes the $n$-fold Kronecker product (or tensor product) of a row stochastic matrix $A$, which corresponds to $n$ uses of the memoryless channel $A \cdot{ }^{12}$ Thus, we have a Markov chain $\tilde{X}_{1}^{n} \rightarrow V_{1}^{n} \rightarrow \tilde{W}_{1}^{n} \rightarrow W_{1}^{n}$ using Definition 2 (where we neglect the difference between physical and stochastic degradation since it is inconsequential in this context). By the data processing inequality, this implies that

$$
I\left(\tilde{X}_{1}^{n} ; W_{1}^{n}\right) \leq I\left(\tilde{X}_{1}^{n} ; \tilde{W}_{1}^{n}\right) .
$$

Fourth, we again swap the random permutation and DMC blocks in the Markov chain $\tilde{X}_{1}^{n} \rightarrow V_{1}^{n} \rightarrow \tilde{W}_{1}^{n}$ using Lemma 2. As we argued earlier, this produces an equivalent Markov chain $\tilde{X}_{1}^{n} \rightarrow \tilde{Z}_{1}^{n} \rightarrow \tilde{Y}_{1}^{n}$ such that the channel $P_{\tilde{Y}_{1}^{n} \mid \tilde{X}_{1}^{n}}$ is equivalent to the channel $P_{\tilde{W}_{1}^{n} \mid \tilde{X}_{1}^{n}}$. Moreover, in this alternative Markov chain, $\tilde{X}_{1}^{n} \sim P_{\tilde{X}^{n}}$ as before, the product channel $P_{\tilde{Z}_{1}^{n} \mid \tilde{X}_{1}^{n}}$ is defined by the DMC $P_{\tilde{Z} \mid \tilde{X}}=S_{\delta}$

\footnotetext{
${ }^{12}$ The tensorization property of the degradation preorder is well-known in information theory. For a proof, notice that given any three row stochastic matrices $A, B, C$ (with consistent dimensions so that the ensuing products are legal), if $A=B C$, then $A \otimes A=(B \otimes B)(C \otimes C)$ using the mixedproduct property, where $\otimes$ denotes the Kronecker product.
}

(i.e., a $q-\mathrm{SC}(\delta)$ ) with input and output alphabet $\mathcal{X}^{\prime}$, and the channel $P_{\tilde{Y}_{1}^{n}} \mid \tilde{Z}_{1}^{n}$ is defined by a random permutation channel. Hence, we have

$$
I\left(\tilde{X}_{1}^{n} ; \tilde{W}_{1}^{n}\right)=I\left(\tilde{X}_{1}^{n} ; \tilde{Y}_{1}^{n}\right) .
$$

Finally, combining (61), (62), (63), and (64), we get

$$
I\left(X_{1}^{n} ; Y_{1}^{n}\right) \leq I\left(\tilde{X}_{1}^{n} ; \tilde{Y}_{1}^{n}\right),
$$

which implies that the right hand side of (57) can be upper bounded as

$$
R \log (n) \leq 1+P_{\text {error }}^{(n)} R \log (n)+I\left(\tilde{X}_{1}^{n} ; \tilde{Y}_{1}^{n}\right) .
$$

Executing the Fisher-Neyman factorization argument from the outset of the proof of Theorem 2, we obtain that $\hat{P}_{\tilde{Y}_{1}^{n}}$ is a sufficient statistic of $\tilde{Y}_{1}^{n}$ for $\tilde{X}_{1}^{n}$. So, we have

$$
R \log (n) \leq 1+P_{\text {error }}^{(n)} R \log (n)+I\left(\tilde{X}_{1}^{n} ; \hat{P}_{\tilde{Y}_{1}^{n}}\right),
$$

much like the bound in (47). At this stage, noting that the DMC $P_{\tilde{Z} \mid \tilde{X}}=S_{\delta}$ is strictly positive, we can upper bound $I\left(\tilde{X}_{1}^{n} ; \hat{P}_{\tilde{Y}_{1}^{n}}\right)$ by following the proof of Theorem 2 mutatis mutandis. Indeed, starting from (65) and proceeding with the proof of Theorem 2 yields

$$
R \leq \frac{q-1}{2}=\frac{\left|\mathcal{X}^{\prime}\right|-1}{2}=\frac{\operatorname{ext}\left(P_{Z \mid X}\right)-1}{2} .
$$

This completes the proof.

\section{Noisy Permutation Channel Capacity}

To complement our main result in Theorem 4, we characterize and bound the noisy permutation channel capacities of several other simple classes of DMCs in this section.

\section{A. Unit Rank Channels}

We start with what is perhaps the simplest setting - that of an "independent channel." In this case, the noisy permutation channel capacity is obviously zero, and a standard Fano's inequality argument rigorously justifies this.

Proposition 3 ( $C_{\text {perm }}$ of Unit Rank Stochastic Matrices). For a unit rank $D M C P_{Z \mid X} \in \mathbb{R}^{|\mathcal{X}| \times|\mathcal{Y}|}$ such that all rows of $P_{Z \mid X}$ are equal, we have

$$
C_{\text {perm }}\left(P_{Z \mid X}\right)=0 .
$$

Proof. We need only prove a converse bound to establish this. Since all rows of $P_{Z \mid X}$ are equal, the output $Z$ of the DMC $P_{Z \mid X}$ is independent of the input $X$. Recalling the setup in subsection I-B, this implies that

$$
I\left(X_{1}^{n} ; \hat{P}_{Y_{1}^{n}}\right)=0 .
$$

Notice that (47) (in the proof of Theorem 2) holds for any DMC. So, dividing both sides of (47) by $\log (n)$ and applying (66) yields

$$
R \leq \frac{1}{\log (n)}+P_{\text {error }}^{(n)} R,
$$

where letting $n \rightarrow \infty$ produces $R \leq 0$. Therefore, we have $C_{\text {perm }}\left(P_{Z \mid X}\right)=0$. 


\section{B. Permutation Transition Matrices}

Next, we consider the straightforward dual setting of a "perfect channel." In this case, the DMC is an identity channel without loss of generality, which means that the corresponding noisy permutation channel just permutes its input codewords randomly (see subsection I-B). So, intuitively, the maximum number of decodable messages that can be reliably communicated is equal to the number of possible empirical distributions over the alphabet of the DMC (see (67) below). Thus, the noisy permutation channel capacity is clearly characterized by the alphabet size of the DMC. The formal proof is again straightforward, but we include it here for completeness.

Proposition 4 ( $C_{\text {perm }}$ of Permutation Stochastic Matrices). For a DMC $P_{Z \mid X}$ such that $|\mathcal{X}|=|\mathcal{Y}|=k \geq 2$ and $P_{Z \mid X} \in \mathbb{R}^{k \times k}$ is a permutation matrix, we have

$$
C_{\text {perm }}\left(P_{Z \mid X}\right)=k-1 .
$$

\section{Proof.}

Achievability: Under the setup of subsection I-B, consider the obvious encoder-decoder pair:

1) The message set $\mathcal{M}=\left\{m=\left[m_{1} \cdots m_{k}\right]^{\mathrm{T}} \in(\mathbb{N} \cup\right.$ $\left.\{0\})^{k}: m_{1}+\cdots+m_{k}=n\right\}$ with cardinality

$$
|\mathcal{M}|=\left(\begin{array}{c}
n+k-1 \\
k-1
\end{array}\right)=\Theta\left(n^{k-1}\right),
$$

where the elements of $\mathcal{M}$ have been re-indexed for convenience.

2) The encoder $f_{n}: \mathcal{M} \rightarrow \mathcal{X}^{n}$ is given by

$$
\forall m \in \mathcal{M}, \quad f_{n}(m)=(\underbrace{1, \ldots, 1}_{m_{1} 1 \text { 's }}, \underbrace{2, \ldots, 2}_{m_{2} 2 \text { 's }}, \ldots, \underbrace{k, \ldots, k}_{m_{k} k^{\prime} \mathrm{s}}),
$$

where $\mathcal{X}=\{1, \ldots, k\}$ without loss of generality.

3) The decoder $g_{n}: \mathcal{Y}^{n} \rightarrow \mathcal{M}$ is given by

$$
\forall y_{1}^{n} \in \mathcal{Y}^{n}, g_{n}\left(y_{1}^{n}\right)=n P_{Z \mid X}\left[\hat{P}_{y_{1}^{n}}(1) \cdots \hat{P}_{y_{1}^{n}}(k)\right]^{\mathrm{T}},
$$

where $\mathcal{Y}=\mathcal{X}$ without loss of generality (and $P_{Z \mid X} \in$ $\mathbb{R}^{k \times k}$ is a permutation matrix).

Clearly, this encoder-decoder pair achieves $P_{\text {error }}^{(n)}=0$. Hence, using (67), the rate

$$
R=\lim _{n \rightarrow \infty} \frac{\log \left(\left(\begin{array}{c}
n+k-1 \\
k-1
\end{array}\right)\right)}{\log (n)}=k-1
$$

is achievable, and $C_{\text {perm }}\left(P_{Z \mid X}\right) \geq k-1$.

Converse: Recall that (47) (in the proof of Theorem 2) holds for any DMC. We bound the mutual information term in (47) with

$$
\begin{aligned}
I\left(X_{1}^{n} ; \hat{P}_{Y_{1}^{n}}\right) & =H\left(\hat{P}_{Y_{1}^{n}}\right) \\
& \leq(k-1) \log (n+1),
\end{aligned}
$$

where the equality holds because $H\left(\hat{P}_{Y_{1}^{n}} \mid X_{1}^{n}\right)=0$, and the inequality uses the upper bound on the number of possible empirical distributions given in (5). Then, as before, combining
(47) with the above bound on mutual information and dividing by $\log (n)$ yields

$$
R \leq \frac{1}{\log (n)}+P_{\text {error }}^{(n)} R+\frac{(k-1) \log (n+1)}{\log (n)},
$$

where letting $n \rightarrow \infty$ produces $R \leq k-1$. Therefore, we have $C_{\text {perm }}\left(P_{Z \mid X}\right) \leq k-1$, which completes the proof.

\section{Strictly Positive Channels}

Recall that Theorem 4 in section II presents the main contribution of this paper-a closed-form expression for the noisy permutation channel capacity of strictly positive DMCs with full rank. For general strictly positive DMCs, we complement Theorem 4 by proposing the following conjecture.

Conjecture 1 ( $C_{\text {perm }}$ of Strictly Positive Channels). For any strictly positive DMC $P_{Z \mid X}$, we have

$$
\begin{aligned}
C_{\text {perm }}\left(P_{Z \mid X}\right) & =\liminf _{n \rightarrow \infty} \frac{1}{\log (n)} \sup _{P_{X_{1}^{n}} \in \mathcal{P}_{\mathcal{X}^{n}}} I\left(\hat{P}_{X_{1}^{n}} ; \hat{P}_{Y_{1}^{n}}\right) \\
& =\frac{\operatorname{rank}\left(P_{Z \mid X}\right)-1}{2},
\end{aligned}
$$

where the supremum in the first equality is over all probability distributions in $\mathcal{P}_{\mathcal{X}^{n}}$, or equivalently, over all probability distributions of $\hat{P}_{X_{1}^{n}}$.

While Definition 1 provides an operational definition of $C_{\text {perm }}\left(P_{Z \mid X}\right)$, the first equality in Conjecture 1 can be construed as the corresponding notion of "information capacity" (analogous to the definition of multi-letter information capacity in, e.g., [19, Definition 18.6]), and the second equality in Conjecture 1 is a closed-form expression for the noisy permutation channel capacity. As Conjecture 1 reveals, we believe that our achievability bound in Theorem 1 is most likely tight. To briefly elaborate on this further, the first equality is inspired by the modified Fano's inequality argument in (47), where we also use Lemma 2 to obtain $I\left(\hat{P}_{X_{1}^{n}} ; \hat{P}_{Y_{1}^{n}}\right)$ instead of $I\left(X_{1}^{n} ; \hat{P}_{Y_{1}^{n}}\right)$, and the second equality is suggested by the first equality via intuition from the multivariate CLT.

Next, as a concrete and canonical illustration of Propositions 3 and 4 and Theorem 4, we present the noisy permutation channel capacity of binary symmetric channels below (see Definition 3 for a definition of $q-\mathrm{SCs}$ ). This result was first proved in [2, Theorem 3]. (Note that in the context of the work in [10], [11], and [12], this BSC setting corresponds to noisy permutation channels with substitution errors.)

Proposition 5 ( $C_{\text {perm }}$ of BSCs [2, Theorem 3]).

$$
C_{\text {perm }}(\operatorname{BSC}(\delta))= \begin{cases}1, & \text { for } \delta \in\{0,1\} \\ \frac{1}{2}, & \text { for } \delta \in\left(0, \frac{1}{2}\right) \cup\left(\frac{1}{2}, 1\right) . \\ 0, & \text { for } \delta=\frac{1}{2}\end{cases}
$$

Proof. The $\delta=\frac{1}{2}$ case follows from Proposition 3, the $\delta \in$ $\{0,1\}$ case follows from Proposition 4 , and the remaining case follows from Theorem 4 .

We remark that Proposition 5 illustrates a few somewhat surprising facts about noisy permutation channel capacity. 
While traditional channel capacity is convex as a function of the channel (with fixed dimensions), noisy permutation channel capacity is clearly non-convex and discontinuous as a function the channel. Moreover, for the most part, the noisy permutation channel capacity of a BSC does not depend on $\delta$. Looking at the proof of Proposition 2 in subsection III-B, this is because the scaling with $n$ of the $\ell^{2}$-distance between two encoded messages does not change after passing through the memoryless BSC (see, e.g., (38) and footnote 8). However, (36) and (43) suggest that $\delta$ does affect the rate of decay of $P_{\text {error }}^{(n)}$. Finally, we note that in a manner similar to Proposition 5, we can also determine the noisy permutation channel capacity of any $q-\mathrm{SC}(\delta)$ for $\delta \in[0,1)$ using Propositions 3 and 4 and Theorem 4 .

\section{Erasure Channels and Doeblin Minorization}

In this subsection, we consider the important class of $q$ ary erasure channels. Indeed, in the context of communication networks, networks where packets can be dropped are typically modeled as noisy permutation channels with possible deletions, or equivalently, erasures, cf. [10], [11], [12, Remark 1]. Since the transition kernels of $q$-ary erasure channels contain zero entries, our converse results in Theorems 2 and 3 do not hold. So, we will present some bounds on the their noisy permutation channel capacities. First, let us recall the definition of $q$-ary erasure channels.

Definition 4 ( $q$-ary Erasure Channel). Under the formalism presented in subsection I-B, we define a q-ary erasure channel $P_{Z \mid X}$ with erasure probability $\eta \in[0,1]$, input alphabet $\mathcal{X}$ with $|\mathcal{X}|=q \in \mathbb{N} \backslash\{1\}$, and output alphabet $\mathcal{Y}=\mathcal{X} \cup\{\mathrm{E}\}$, where $\mathrm{E}$ denotes the erasure symbol, using the conditional distributions

$$
\forall z \in \mathcal{Y}, \forall x \in \mathcal{X}, P_{Z \mid X}(z \mid x)= \begin{cases}1-\eta, & \text { for } z=x \\ \eta, & \text { for } z=\mathrm{E} \\ 0, & \text { otherwise }\end{cases}
$$

Moreover, we represent such a channel $P_{Z \mid X}$ as $q$-EC $(\eta)$ for convenience.

We note that in the special case where $q=2, \mathcal{X}=\{0,1\}$, and $\eta$ is the probability that the input bit is erased, we refer to the $2-\mathrm{SC}(\eta)$ as a binary erasure channel (BEC), denoted $\operatorname{BEC}(\eta)$.

Next, in order to present our bounds on the noisy permutation channel capacity of erasure channels, we introduce a classical concept from the Markov process literature. As we will see, one approach to proving our achievability bound entails using a symmetric channel that is degraded by the erasure channel under consideration. While we have introduced degradation in subsection III-A, the specific setting of degradation by erasure channels has been studied extensively in the Markov process literature under the guise of "Doeblin minorization." We next introduce the concept of Doeblin minorization in an information theoretic light (within the formalism of subsection I-B), cf. [52, Section 3].

Definition 5 (Doeblin Minorization). A row stochastic matrix $P_{Z \mid X} \in \mathbb{R}^{|\mathcal{X}| \times|\mathcal{Y}|}$ satisfies the Doeblin minorization condition if there exists a probability distribution $Q_{Z} \in \mathcal{P}_{\mathcal{Y}}$ and a constant $\eta \in(0,1)$ such that

$$
\forall z \in \mathcal{Y}, \forall x \in \mathcal{X}, \quad P_{Z \mid X}(z \mid x) \geq \eta Q_{Z}(z),
$$

and we say that $P_{Z \mid X}$ satisfies Doeblin $\left(Q_{Z}, \eta\right)$. Furthermore, we say that $P_{Z \mid X}$ satisfies Doeblin $\left(Q_{Z}, 0\right)$ when $P_{Z \mid X}$ does not satisfy the Doeblin minorization condition (since the above condition is trivially true when $\eta=0$ ).

Definition 5 of Doeblin minorization is less general than its definition in a finite state space Markov chain context, where one often studies "local minorization" of multi-step Markov transition kernels, cf. [52, Section 4]. On the other hand, our definition applies to more general (rectangular) transition kernels. While the Doeblin minorization condition was originally developed to study the ergodicity of Markov processes, ${ }^{13}$ as we alluded to earlier, it turns out to be equivalent to degradation by an erasure channel. The next lemma depicts this known, but seemingly overlooked, connection.

Lemma 6 (Doeblin Minorization and Degradation [52], [55]). Consider any DMC $P_{Z \mid X}$ with input alphabet $\mathcal{X}$ and output alphabet $\mathcal{Y}$ with $|\mathcal{X}|=q$. Then, the following are true:

1) (Equivalence [52, Theorem 3.1]) For any constant $\eta \in$ $(0,1), P_{Z \mid X}$ satisfies Doeblin $\left(Q_{Z}, \eta\right)$ for some distribution $Q_{Z} \in \mathcal{P}_{\mathcal{Y}}$ if and only if $P_{Z \mid X}$ is a degraded version of the $q$-ary erasure channel $q-\mathrm{EC}(\eta)$.

2) (Extremality [55, Lemma 4]) The extremal erasure probability $\eta_{*}=\eta_{*}\left(P_{Z \mid X}\right)$ such that $P_{Z \mid X}$ is a degraded version of $q-\mathrm{EC}\left(\eta_{*}\right)$ is given by

$$
\begin{aligned}
\eta_{*} & \triangleq \max \left\{\eta \in[0,1]: \begin{array}{l}
P_{Z \mid X} \text { is a degraded } \\
\text { version of } q-\mathrm{EC}(\eta)
\end{array}\right\} \\
& =\sup \left\{\eta \in[0,1): \begin{array}{l}
P_{Z \mid X} \text { satisfies Doeblin }\left(Q_{Z}, \eta\right) \\
\text { for some distribution } Q_{Z} \in \mathcal{P}_{\mathcal{Y}}
\end{array}\right\} \\
& =\sum_{z \in \mathcal{Y}} \min _{x \in \mathcal{X}} P_{Z \mid X}(z \mid x),
\end{aligned}
$$

where the second equality follows from part 1 and the quantity in the final equality is known as Doeblin's coefficient of ergodicity, $c f$. [56, Definition 5.1].

Although Lemma 6 is known in the literature, we provide a proof of part 1 in appendix D for completeness. Moreover, we note that the equivalent description of Doeblin minorization as degradation by an erasure channel can also be viewed as a specialization of the so called regeneration or Nummelin splitting technique in the theory of Harris chains [57], [58].

In the ensuing theorem, we derive Theorem 5, which uses the notion of degradation to prove a comparison bound for noisy permutation channel capacities, as well as a related bound pertaining to Doeblin minorization, which specializes Theorem 5 for erasure channels (as revealed by our discussion heretofore). As outlined in subsection II-D, this result concurs with the intuition that degraded channels are "more noisy," and therefore, have smaller noisy permutation channel capacity.

\footnotetext{
${ }^{13}$ As a historical remark, it is worth mentioning that as stated in [52, Section 3], "two of the most powerful ideas in the modern theory of Markov processes were introduced [by Doeblin in [53] and [54]]; namely minorization and coupling, respectively."
} 
Theorem 6 (Comparison Bounds via Degradation). Consider any two DMCs $P_{Z_{1} \mid X} \in \mathbb{R}^{|\mathcal{X}| \times\left|\mathcal{Z}_{1}\right|}$ and $P_{Z_{2} \mid X} \in \mathbb{R}^{|\mathcal{X}| \times\left|\mathcal{Z}_{2}\right|}$, with common input alphabet $\mathcal{X}$ and output alphabets $\mathcal{Z}_{1}$ and $\mathcal{Z}_{2}$, respectively. Then, the following are true:

1) If $P_{Z_{2} \mid X}$ is a degraded version of $P_{Z_{1} \mid X}$, then we have

$$
C_{\text {perm }}\left(P_{Z_{2} \mid X}\right) \leq C_{\text {perm }}\left(P_{Z_{1} \mid X}\right) .
$$

2) If $P_{Z_{2} \mid X}$ satisfies Doeblin $\left(Q_{Z}, \eta\right)$ for some distribution $Q_{Z} \in \mathcal{P}_{\mathcal{Y}}$ and some constant $\eta \in(0,1)$, then we have

$$
C_{\text {perm }}\left(P_{Z_{2} \mid X}\right) \leq C_{\text {perm }}(q-\mathrm{EC}(\eta)),
$$

where we let $q=|\mathcal{X}|$.

Proof.

Part 1: Recalling the formalism introduced in subsection I-B, fix any (small) $\epsilon>0$ such that $R \triangleq C_{\text {perm }}\left(P_{Z_{2} \mid X}\right)-\epsilon \geq 0$ is an achievable rate for the DMC $P_{Z_{2} \mid X}$. Then, for the noisy permutation channel model with DMC $P_{Z_{2} \mid X}$, consider the Markov chain $M \rightarrow f_{n}(M)=X_{1}^{n} \rightarrow\left(Z_{2}\right)_{1}^{n} \rightarrow\left(Y_{2}\right)_{1}^{n} \rightarrow$ $g_{n}\left(\left(Y_{2}\right)_{1}^{n}\right)$, defined by a message set $\mathcal{M}$ with cardinality $|\mathcal{M}|=n^{R}$, a sequence of possibly randomized encoders $\left\{f_{n}: \mathcal{M} \rightarrow \mathcal{X}^{n}\right\}_{n \in \mathbb{N}}$, and a sequence of associated possibly randomized decoders $\left\{g_{n}: \mathcal{Z}_{2}^{n} \rightarrow \mathcal{M} \cup\{\mathrm{e}\}\right\}_{n \in \mathbb{N}}$, where $\left(Y_{2}\right)_{1}^{n} \in \mathcal{Z}_{2}^{n}$ denotes a random permutation of the output codeword $\left(Z_{2}\right)_{1}^{n}$ of the DMC $P_{Z_{2} \mid X}$. Let us define

$$
P_{\text {error }}^{(n)}\left(P_{Z_{2} \mid X}, f_{n}, g_{n}\right) \triangleq \mathbb{P}\left(M \neq g_{n}\left(\left(Y_{2}\right)_{1}^{n}\right)\right)
$$

as the average probability of error for the noisy permutation channel model corresponding to $P_{Z_{2} \mid X}, f_{n}$, and $g_{n}$. Since $R$ is an achievable rate, we further assume that $f_{n}$ and $g_{n}$ are chosen such that $\lim _{n \rightarrow \infty} P_{\text {error }}^{(n)}\left(P_{Z_{2} \mid X}, f_{n}, g_{n}\right)=0$. By our assumption in the theorem statement, we know using Definition 2 that there exists some DMC $P_{Z_{2} \mid Z_{1}} \in \mathbb{R}^{\left|\mathcal{Z}_{1}\right| \times\left|\mathcal{Z}_{2}\right|}$ (with input alphabet $\mathcal{Z}_{1}$ and output alphabet $\mathcal{Z}_{2}$ ) such that $P_{Z_{2} \mid X}=P_{Z_{1} \mid X} P_{Z_{2} \mid Z_{1}}$. We will use this degradation relation to construct a "good" encoder-decoder pair for the DMC $P_{Z_{1} \mid X}$

To this end, for the noisy permutation channel model with DMC $P_{Z_{1} \mid X}$, consider the Markov chain $M \rightarrow f_{n}(M)=$ $X_{1}^{n} \rightarrow\left(Z_{1}\right)_{1}^{n} \rightarrow\left(Y_{1}\right)_{1}^{n}$, where we use the same message set (with cardinality $n^{R}$ ) and encoder sequence as before, and $\left(Y_{1}\right)_{1}^{n} \in \mathcal{Z}_{1}^{n}$ is a random permutation of the output codeword $\left(Z_{1}\right)_{1}^{n}$ of the DMC $P_{Z_{1} \mid X}$. (In fact, the random variables $M$ and $X_{1}^{n}$ are coupled to be equal for the two models.) Now, for every $n \in \mathbb{N}$, construct the decoder $\tilde{g}_{n}: \mathcal{Z}_{1}^{n} \rightarrow \mathcal{M} \cup\{\mathrm{e}\}$ so that

$$
\forall y_{1}^{n} \in \mathcal{Z}_{1}^{n}, \quad \tilde{g}_{n}\left(y_{1}^{n}\right) \triangleq g_{n}\left(Z_{1}^{n}\right),
$$

where $Z_{i} \sim P_{Z_{2} \mid Z_{1}}\left(\cdot \mid y_{i}\right)$ for every $i \in\{1, \ldots, n\}$, and $Z_{1}^{n}$ are mutually independent. This produces the Markov chain $M \rightarrow X_{1}^{n} \rightarrow\left(Z_{1}\right)_{1}^{n} \rightarrow\left(Y_{1}\right)_{1}^{n} \rightarrow \tilde{g}_{n}\left(\left(Y_{1}\right)_{1}^{n}\right)$. We note that the decoder in (68) essentially "simulates" the auxiliary DMC $P_{Z_{2} \mid Z_{1}}$ so that its output is statistically equivalent to the output of the decoder $g_{n}$ with DMC $P_{Z_{2} \mid X}$.

We next prove the intuitively straightforward relation

$$
P_{\text {error }}^{(n)}\left(P_{Z_{2} \mid X}, f_{n}, g_{n}\right)=P_{\text {error }}^{(n)}\left(P_{Z_{1} \mid X}, f_{n}, \tilde{g}_{n}\right) .
$$

To establish this, consider yet another Markov chain, $M \rightarrow$ $f_{n}(M)=X_{1}^{n} \rightarrow V_{1}^{n} \rightarrow W_{1}^{n} \rightarrow U_{1}^{n}$, where the message set and $f_{n}$ are the same as before, $V_{1}^{n} \in \mathcal{X}^{n}$ is a random permutation of $X_{1}^{n}, W_{1}^{n} \in \mathcal{Z}_{1}^{n}$ is the output of the DMC $P_{Z_{1} \mid X}$ with input $V_{1}^{n}$, and $U_{1}^{n} \in \mathcal{Z}_{2}^{n}$ is the output of the DMC $P_{Z_{2} \mid Z_{1}}$ with input $W_{1}^{n}$. Using Lemma 2, notice that the conditional distribution $P_{\left(Y_{1}\right)_{1}^{n} \mid X_{1}^{n}}$ is equivalent to the conditional distribution $P_{W_{1}^{n} \mid X_{1}^{n}}$. Furthermore, Lemma 2 and the degradation relation $P_{Z_{2} \mid X}=P_{Z_{1} \mid X} P_{Z_{2} \mid Z_{1}}$ imply that the conditional distribution $P_{\left(Y_{2}\right)_{1}^{n} \mid X_{1}^{n}}$ is equivalent to the conditional distribution $P_{U_{1}^{n} \mid X_{1}^{n}}$. Thus, the joint distribution of $\left(M, \tilde{g}_{n}\left(\left(Y_{1}\right)_{1}^{n}\right)\right)$ is equal to the joint distribution of $\left(M, g_{n}\left(\left(Y_{2}\right)_{1}^{n}\right)\right)$, because the conditional distributions of $\tilde{g}_{n}\left(W_{1}^{n}\right)$ and $g_{n}\left(U_{1}^{n}\right)$ given $M$ are equivalent, where we may perceive $U_{1}^{n}$ as the intermediate random variables used by $\tilde{g}$ in (68) so that $\tilde{g}_{n}\left(W_{1}^{n}\right)=g_{n}\left(U_{1}^{n}\right)$. This produces the relation (69).

Lastly, we conclude this proof by realizing that (69) reveals that $R=C_{\text {perm }}\left(P_{Z_{2} \mid X}\right)-\epsilon$ is an achievable rate for the DMC $P_{Z_{1} \mid X}$. Therefore, we have

$$
C_{\text {perm }}\left(P_{Z_{2} \mid X}\right)-\epsilon \leq C_{\text {perm }}\left(P_{Z_{1} \mid X}\right),
$$

and we can let $\epsilon \rightarrow 0$ to obtain the desired inequality.

Part 2: This follows immediately from part 1 of this theorem and Lemma 6.

We are now in a position to present bounds on the noisy permutation channel capacity of $q$-ary erasure channels. Although the achievability bound in the ensuing proposition can be obtained as a direct consequence of Theorem 1, we will elucidate an alternative coding scheme that establishes this bound using Doeblin minorization. Similarly, although the converse bound in the ensuing proposition is just the trivial bound given in (11), we will provide an alternative proof for it.

Proposition 6 (Bounds on $C_{\text {perm }}$ of $q$-EC). For a $q$-ary erasure channel $q-\mathrm{EC}(\eta)$ with $\eta \in(0,1)$, we have

$$
\frac{q-1}{2} \leq C_{\text {perm }}(q-\mathrm{EC}(\eta)) \leq q-1 .
$$

Furthermore, the extremal noisy permutation channel capacities are $C_{\text {perm }}(q-\mathrm{EC}(0))=q-1$ and $C_{\text {perm }}(q-\mathrm{EC}(1))=0$.

Proof.

Achievability for $\eta \in(0,1)$ : To derive a lower bound on $C_{\text {perm }}(q-\mathrm{EC}(\eta))$, we will employ a useful representation of $q$-SC's using $q$-EC's. Observe that the row stochastic transition probability matrix of a $q-\mathrm{SC}(\eta(q-1) / q)$ can be decomposed as

$$
S_{\eta(q-1) / q}=(1-\eta) I+\eta\left(\frac{1}{q} \mathbf{1 1}^{\mathrm{T}}\right),
$$

where $S_{\eta(q-1) / q}$ is the $q$-ary symmetric channel matrix defined in (13), $I \in \mathbb{R}^{q \times q}$ is the identity matrix representing a channel that exactly copies its input, $\mathbf{1}=[1 \cdots 1]^{\mathrm{T}} \in \mathbb{R}^{q}$ denotes the column vector with all elements equal to unity, and $\frac{1}{q} 11^{\mathrm{T}}$ represents a channel whose output is an independent uniform random variable. Hence, a $q-\mathrm{SC}(\eta(q-1) / q)$ can be equivalently construed as a channel that either copies its input random variable with probability $1-\eta$, or generates a completely independent output random variable that is uniformly 
distributed on the input alphabet $\mathcal{X}$ (where $|\mathcal{X}|=q$ ) with probability $\eta$. A consequence of this interpretation, or equivalently, the decomposition (70), is that a $q-\mathrm{SC}(\eta(q-1) / q)$ satisfies $\operatorname{Doeblin}\left(\mathbf{1}^{\mathrm{T}} / q, \eta\right)$, where $\mathbf{1}^{\mathrm{T}} / q$ is a row vector representing the uniform distribution. Using part 1 of Lemma 6, this means that a $q-\mathrm{SC}(\eta(q-1) / q)$ is a degraded version of a $q-\mathrm{EC}(\eta)$; in particular, a $q-\mathrm{SC}(\eta(q-1) / q)$ is statistically equivalent to a $q$ - $\mathrm{EC}(\eta)$ followed by a channel that outputs an independent uniformly distributed random variable for the input erasure symbol E, and copies all other input symbols. Moreover, part 2 of Theorem 6 conveys that

$$
C_{\text {perm }}\left(q-\mathrm{SC}\left(\frac{\eta(q-1)}{q}\right)\right) \leq C_{\text {perm }}(q-\mathrm{EC}(\eta)) .
$$

Since $\frac{\eta(q-1)}{q} \in\left(0, \frac{q-1}{q}\right)$, it is straightforward to verify that the $q$-ary symmetric channel matrix $S_{\eta(q-1) / q}$ is strictly positive and non-singular. So, we have

$$
C_{\text {perm }}(q-\mathrm{EC}(\eta)) \geq C_{\text {perm }}\left(q-\mathrm{SC}\left(\frac{\eta(q-1)}{q}\right)\right)=\frac{q-1}{2}
$$

using Theorem 4, which proves the desired result.

We remark that according to the proof of part 1 of Theorem 6 , an appropriately altered coding scheme from the achievability proof of Theorem 1 in subsection III-B, which has:

1) A randomized encoder described by (27),

2) A decoder that first generates independent uniform random output letters to replace every erasure symbol in the output codeword, and then applies the decoder (29), which is characterized by (30) specialized to a $q-\mathrm{SC}(\eta(q-1) / q)$,

achieves the lower bound on $C_{\text {perm }}(q-\mathrm{EC}(\eta))$. Alternatively, if we directly use Theorem 1 and the fact that $\operatorname{rank}(q-\mathrm{EC}(\eta))=$ $q$ to obtain the lower bound on $C_{\text {perm }}(q-\mathrm{EC}(\eta))$ (as mentioned earlier), then this corresponds to using the same encoder (27), but an alternative decoder (29), which is characterized by (30) specialized to a $q-\mathrm{EC}(\eta)$.

Converse for $\eta \in(0,1)$ : As mentioned earlier, the upper bound in the proposition statement is immediate from (11) and the fact that $\operatorname{ext}(q-\mathrm{EC}(\eta))=q$. However, as outlined after (11) in subsection II-B, the inequality in terms of ext $(\cdot)$ in (11) is proved using a degradation argument akin to the proof of Theorem 3. Here, we provide a simpler alternative proof of the (intuitively obvious) upper bound in the proposition statement by exploiting specific properties of $q$-ary erasure channels.

Recall that (47) (from the proof of Theorem 2) holds for a $q-\mathrm{EC}(\eta)$, and we can bound the mutual information term in (47) via

$$
\begin{aligned}
& I\left(X_{1}^{n} ; \hat{P}_{Y_{1}^{n}}\right) \stackrel{(\mathrm{a})}{=} I\left(X_{1}^{n} ; \hat{P}_{Y_{1}^{n}}(\mathrm{E}), \hat{P}_{Y_{1}^{n}}(1), \ldots, \hat{P}_{Y_{1}^{n}}(q-1)\right) \\
& \stackrel{\text { (b) }}{=} I\left(X_{1}^{n} ; \hat{P}_{Y_{1}^{n}}(1), \ldots, \hat{P}_{Y_{1}^{n}}(q-1) \mid \hat{P}_{Y_{1}^{n}}(\mathrm{E})\right) \\
& +I\left(X_{1}^{n} ; \hat{P}_{Y_{1}^{n}}(\mathrm{E})\right) \\
& \stackrel{\text { (c) }}{=} I\left(X_{1}^{n} ; \hat{P}_{Y_{1}^{n}}(1), \ldots, \hat{P}_{Y_{1}^{n}}(q-1) \mid \hat{P}_{Y_{1}^{n}}(\mathrm{E})\right) \\
& =H\left(\hat{P}_{Y_{1}^{n}}(1), \ldots, \hat{P}_{Y_{1}^{n}}(q-1) \mid \hat{P}_{Y_{1}^{n}}(\mathrm{E})\right) \\
& -H\left(\hat{P}_{Y_{1}^{n}}(1), \ldots, \hat{P}_{Y_{1}^{n}}(q-1) \mid X_{1}^{n}, \hat{P}_{Y_{1}^{n}}(\mathrm{E})\right) \\
& \stackrel{(\mathrm{d})}{\leq}(q-1) \log (n+1)
\end{aligned}
$$

$$
-H\left(\hat{P}_{Y_{1}^{n}}(1), \ldots, \hat{P}_{Y_{1}^{n}}(q-1) \mid X_{1}^{n}, \hat{P}_{Y_{1}^{n}}(\mathrm{E})\right)
$$

(e)

$$
\stackrel{\text { e) }}{\leq}(q-1) \log (n+1)
$$

where (a) holds because $\hat{P}_{Y_{1}^{n}}$ sums to unity and we let $\mathcal{X}=\{1, \ldots, q\}$ without loss of generality, (b) follows from the chain rule, (c) uses the fact that $I\left(X_{1}^{n} ; \hat{P}_{Y_{1}^{n}}(\mathrm{E})\right)=0$ since the codeword $X_{1}^{n}$ is independent of the number of erasures $n \hat{P}_{Y_{1}^{n}}(\mathrm{E})$, (d) holds because $n \hat{P}_{Y_{1}^{n}}(i) \in\{0, \ldots, n\}$ for every $i \in\{1, \ldots, q-1\}$, and (e) follows from the non-negativity of Shannon entropy. Therefore, as before, substituting (72) into (47), dividing by $\log (n)$, and letting $n \rightarrow \infty$, we get that any achievable rate $R \geq 0$ satisfies $R \leq q-1$. This proves that $C_{\text {perm }}(q-\mathrm{EC}(\eta)) \leq q-1$.

Case $\eta=0$ : In this case, the $q$-EC $(0)$ is just the deterministic identity channel. Hence, $C_{\text {perm }}(q-\mathrm{EC}(0))=q-1$ using Proposition 4.

Case $\eta=1$ : In this case, the $q$-EC(1) erases all its input symbols so that we obtain an "independent channel." Hence, $C_{\text {perm }}(q-\mathrm{EC}(1))=0$ using Proposition 3.

We finally make several pertinent remarks. Firstly, in the special case of $q=2$ and $\eta \in(0,1)$, the elegant interpretation of a $\operatorname{BSC}\left(\frac{\eta}{2}\right)$ as a $\operatorname{BEC}(\eta)$ which additionally replaces any output erasure symbol $E$ with an independent $\operatorname{Ber}\left(\frac{1}{2}\right)$ bit, or equivalently, the decomposition (70), is a notion that originates from Fortuin-Kasteleyn random cluster representations of Ising models in the study of percolation, cf. [59]. Moreover, this notion has been exploited in various other discrete probability contexts such as reliable computation using noisy circuits [60, p.570], broadcasting on trees [43, p.412], and broadcasting on directed acyclic graphs [61, Appendix C] (also see [62, p.1634]).

Secondly, in the special case of $q=2$ and $\eta \in(0,1)$, we propose the following conjecture.

Conjecture 2 ( $C_{\text {perm }}$ of BECs). For any erasure probability $\eta \in(0,1)$, the noisy permutation channel capacity of the $\operatorname{BEC}(\eta)$ is given by

$$
C_{\text {perm }}(\operatorname{BEC}(\eta))=\frac{1}{2} .
$$

Specifically, we believe that the achievability bound presented in Proposition 6 is tight. Consequently, unlike traditional channel capacity, we believe that the noisy permutation channel capacities of BSCs and BECs are equal in the nontrivial regimes of their parameters. Indeed, the converse bound in Proposition 6 for the case $q=2, C_{\text {perm }}(\operatorname{BEC}(\eta)) \leq 1$, is intuitively trivial as there are only $n+1$ distinct empirical distributions of codewords in $\{0,1\}^{n}$. So, we anticipate that this bound can be significantly tightened. One approach towards tightening the converse bound would be to consider the mutual information bound in (71), and much like the proof of Theorem 2 in subsection III-C, derive a lower bound on $H\left(\hat{P}_{Y_{1}^{n}}(1) \mid X_{1}^{n}, \hat{P}_{Y_{1}^{n}}(\mathrm{E})\right)$ of the form

$$
H\left(\hat{P}_{Y_{1}^{n}}(1) \mid X_{1}^{n}, \hat{P}_{Y_{1}^{n}}(\mathrm{E})\right) \geq \frac{1}{2} \log (n)+o(\log (n)) .
$$

Clearly, combining (47), (71), and (73) would yield the desired bound $C_{\text {perm }}(\operatorname{BEC}(\eta)) \leq \frac{1}{2}$. As explained in [2, Equation 
(26)], finding a bound of the form (73) corresponds to analyzing the Shannon entropy of hypergeometric distributions in non-trivial regimes of their parameters.

Thirdly, when $q \geq 3$, we also postulate that for any $\eta \in$ $(0,1)$,

$$
C_{\text {perm }}(q-\mathrm{EC}(\eta)) \leq \frac{q}{2} .
$$

While this upper bound does not exactly determine the noisy permutation channel capacity of $q$-ary erasure channels, it does have the following useful corollaries (if proven to be true):

1) In the limit of asymptotically large input alphabet size (i.e., as $q \rightarrow \infty$ ), the noisy permutation channel capacity of $q$-ary erasure channels is characterized by

$$
\forall \eta \in(0,1), \quad \lim _{q \rightarrow \infty} \frac{C_{\text {perm }}(q-\mathrm{EC}(\eta))}{q}=\frac{1}{2} .
$$

2) Applying part 2 of Theorem 6, if a DMC $P_{Z \mid X}$ satisfies the Doeblin minorization condition, then we obtain the converse bound

$$
C_{\text {perm }}\left(P_{Z \mid X}\right) \leq \frac{|\mathcal{X}|}{2}
$$

This bound is clearly weaker than that in Theorem 3 for strictly positive DMCs. However, it also holds for certain DMCs that have zero entries, and therefore, extends Theorem 3 for such DMCs.

We believe (74) could be true, because we can upper bound the mutual information term in (47) so that

$$
\begin{aligned}
I\left(X_{1}^{n} ; \hat{P}_{Y_{1}^{n}}\right) & =H\left(\hat{P}_{Y_{1}^{n}}\right)-H\left(\hat{P}_{Y_{1}^{n}} \mid X_{1}^{n}\right) \\
& \stackrel{(\mathrm{a})}{\leq} q \log (n+1)-H\left(\hat{P}_{Y_{1}^{n}}(1), \ldots, \hat{P}_{Y_{1}^{n}}(q) \mid X_{1}^{n}\right) \\
& \stackrel{(\mathrm{b})}{=} q \log (n+1)-H\left(N_{\mathrm{E}}(1), \ldots, N_{\mathrm{E}}(q) \mid X_{1}^{n}\right) \\
& \stackrel{(\mathrm{c})}{=} q \log (n+1)-\sum_{i=1}^{q} H\left(N_{\mathrm{E}}(i) \mid X_{1}^{n}\right) \\
& \stackrel{(\mathrm{d})}{=} q \log (n+1)-\sum_{i=1}^{q} \mathbb{E}\left[H\left(\operatorname{bin}\left(n \hat{P}_{X_{1}^{n}}(i), \eta\right)\right)\right],
\end{aligned}
$$

where (a) follows from the bound in (5), the fact that $\hat{P}_{Y_{1}^{n}}$ sums to unity, and by letting $\mathcal{X}=\{1, \ldots, q\}$ without loss of generality, (b) follows from defining the number of erasures that occur on the input symbol $i \in \mathcal{X}$ as the random variable $N_{\mathrm{E}}(i) \triangleq n \hat{P}_{X_{1}^{n}}(i)-n \hat{P}_{Y_{1}^{n}}(i) \in\left\{0, \ldots, n \hat{P}_{X_{1}^{n}}(i)\right\}$, (c) holds because $\left\{N_{\mathrm{E}}(i): i \in \mathcal{X}\right\}$ are conditionally independent given $X_{1}^{n}$, (d) holds because each $N_{\mathrm{E}}(i)$ is a binomial random variable with $n \hat{P}_{X_{1}^{n}}(i)$ trials and success probability $\eta$ conditioned on $X_{1}^{n}$, and each term $\mathbb{E}\left[H\left(\operatorname{bin}\left(n \hat{P}_{X_{1}^{n}}(i), \eta\right)\right)\right]$ represents the expectation of a binomial entropy with respect to the distribution of $X_{1}^{n}$. If it can be shown that any encoder, which achieves vanishing probability of error for rates "close to" the noisy permutation channel capacity, must satisfy $\hat{P}_{X_{1}^{n}}(i) \geq \alpha$ for all $i \in \mathcal{X}$ for some constant $\alpha \in(0,1)$ with high probability, then (47), (77), and Lemma 1 would yield (74). However, proving such a lower bound on $\hat{P}_{X_{1}^{n}}$ appears to be challenging (if at all possible), since we essentially have to develop a "probabilistic pigeonhole principle" to argue that "good" encoders need to utilize all the symbols in $\mathcal{X}$ significantly.

\section{CONCLUSION}

In closing, we first briefly reiterate our main contributions. Propelled by existing literature in coding theory, communication networks, and molecular and biological communications, we formulated the information theoretic notion of noisy permutation channel capacity for the problem of reliably transmitting information through a noisy permutation channel, i.e., a DMC followed by an independent random permutation transformation. We then derived achievability and converse bounds on noisy permutation channel capacities in Theorems 1,2 , and 3 (as well as in (11)). These results gave rise to an exact characterization of the noisy permutation channel capacity of strictly positive and full rank DMCs in Theorem 4. Furthermore, in our effort to prove these results and acquire a deeper understanding of noisy permutation channel capacity, we elucidated a simple construction of symmetric channels that dominate given DMCs in the degradation sense in Proposition 1, and established an intuitive monotonicity relation between noisy permutation channel capacity and degradation in Theorem 6.

We next propose some directions for future research. Evidently, addressing any of the open problems explicated in Conjectures 1, 2, and (74) is an excellent starting point to furthering this line of work. After these conjectures are resolved, our ultimate objective is to establish the noisy permutation channel capacity of general DMCs (whose row stochastic matrices can have zero entries). We remark that determining the noisy permutation channel capacities of DMCs with zero entries appears to be more intractable than strictly positive DMCs, because zero entries introduce a combinatorial flavor to the problem. ${ }^{14}$ So, completely settling the noisy permutation channel capacity question for general DMCs is likely to be quite challenging. Finally, there are several other open questions that parallel aspects of classical information theoretic development such as:

1) Finding tight bounds on the average probability of error (akin to classical error exponent analysis), cf. [65, Chapter 5].

2) Developing strong converse results, cf. [19, Section 22.1], [65, Theorem 5.8.5].

3) Establishing exact asymptotics for the maximum achievable value of $|\mathcal{M}|$ (akin to "finite blocklength analysis"), cf. [66], [67, Chapter II.4], and the references therein.

4) Extending the noisy permutation channel model by replacing DMCs with other kinds of memoryless channels or networks, e.g., additive white Gaussian noise (AWGN) channels or multiple-access channels (MACs), and by using more general or "realistic" algebraic operations that are applied to the output codewords, e.g., random permutations that belong to subgroups of the

\footnotetext{
${ }^{14}$ This combinatorial aspect of the problem is similar to (but not exactly the same as) the zero error capacity problem, cf. [63]. It is well-known that calculating the zero error capacity of channels is very challenging, and the best known approaches use semidefinite programming relaxations such as the Lovász $\vartheta$ function, cf. [64].
} 
symmetric group. (For example, when modeling out-oforder delivery of packets in a communication network, all permutations of the packets are not equally likely; indeed, the first two transmitted packets are quite likely to arrive swapped at the receiver, but the first and last transmitted packets are very unlikely to change their relative ordering.)

Altogether, our main results and these future directions illustrate that the study of noisy permutation channel capacity begets a fairly rich, relevant, and seemingly solvable class of new problems.

\section{APPENDiX A \\ PROOF OF PROPOSITION 1}

Proof. To prove this result, we seek to find $q-\mathrm{SC}(\delta)$ 's with $\delta \in\left[0, \frac{q-1}{q}\right]$ such that $P_{Z \mid X}$ is a degraded version of $q-\mathrm{SC}(\delta)$. Indeed, it is straightforward to see that the upper bound on $\delta$ in the proposition statement satisfies

$$
\frac{\nu}{1-\nu+\frac{\nu}{q-1}} \leq \frac{q-1}{q}
$$

because (78) is equivalent to

$$
\frac{\nu(q-1)}{(q-1)-\nu(q-2)} \leq \frac{q-1}{q} \quad \Leftrightarrow \quad \nu \leq \frac{1}{2}
$$

for any $q \in \mathbb{N} \backslash\{1\}$, and the latter bound is always true since $|\mathcal{Y}| \geq 2$. Furthermore, we have equality in (78) if and only if $\nu=\frac{1}{2}$, which happens precisely when $|\mathcal{Y}|=2$ and all rows of $P_{Z \mid X}$ are equal to $\left(\frac{1}{2}, \frac{1}{2}\right)$. In this case, $P_{Z \mid X}=S_{\delta} P_{Z \mid X}$ for all $\delta \in[0,1]$, and the sufficient condition for degradation in the proposition statement holds trivially. So, we will assume without loss of generality that $\nu<\frac{1}{2}$ in the rest of the proof.

To construct $q-\mathrm{SC}(\delta)$ 's with $\delta \in\left[0, \frac{q-1}{q}\right)$ such that $P_{Z \mid X}$ is a degraded version of $q-\mathrm{SC}(\delta)$, we must ensure that $P_{Z \mid X}=$ $S_{\delta} Q$ for some row stochastic matrix $Q \in \mathbb{R}^{q \times|\mathcal{Y}|}$. Equivalently, $S_{\delta}^{-1} P_{Z \mid X}$ must be a row stochastic matrix. A direct calculation yields $S_{\delta}^{-1}=S_{\tau}$ with (cf. [35, Proposition 4])

$$
\tau=\frac{-\delta}{1-\delta-\frac{\delta}{q-1}}
$$

i.e., $S_{\delta}^{-1}=S_{\tau}$ has the structure shown in (13) (but with $\tau$ replacing $\delta$ ). Since the rows of $S_{\tau}$ sum to unity, the rows of $S_{\delta}^{-1} P_{Z \mid X}=S_{\tau} P_{Z \mid X}$ also sum to unity. Thus, it suffices to verify that the minimum entry of $S_{\tau} P_{Z \mid X}$ is non-negative.

For $\delta \in\left[0, \frac{q-1}{q}\right)$, we have $\tau \leq 0$, which means that the principal diagonal elements of $S_{\tau}$ are at least unity, and the offdiagonal elements of $S_{\tau}$ are non-positive. Hence, the minimum entry of $S_{\tau} P_{Z \mid X}$ is lower bounded by

$$
\begin{aligned}
\min _{\substack{i \in\{1, \ldots, q\} \\
j \in\{1, \ldots,|\mathcal{Y}|\}}}\left[S_{\tau} P_{Z \mid X}\right]_{i, j} & \geq(1-\tau) \nu+\tau(1-\nu) \\
& =\frac{\nu-\delta\left(1-\nu+\frac{\nu}{q-1}\right)}{1-\delta-\frac{\delta}{q-1}},
\end{aligned}
$$

where the inequality uses the fact that the maximum entry of $P_{Z \mid X}$ is upper bounded by $1-\nu$ (because $P_{Z \mid X}$ is a stochastic matrix), and the equality follows from substituting (79). So, a sufficient condition that ensures that the minimum entry of $S_{\tau} P_{Z \mid X}$ is non-negative is

$$
\frac{\nu-\delta\left(1-\nu+\frac{\nu}{q-1}\right)}{1-\delta-\frac{\delta}{q-1}} \geq 0 \quad \Leftrightarrow \quad \delta \leq \frac{\nu}{1-\nu+\frac{\nu}{q-1}} .
$$

This completes the proof.

\section{APPENDIX B}

PROOF OF LEMMA 4

Proof. For the binary hypothesis problem in (17), define the "translated empirical distribution of $X_{1}^{n}$ " random vector

$$
T_{n} \triangleq \hat{P}_{X_{1}^{n}}-C_{n} \in \mathcal{T}_{n},
$$

where the constant vector $C_{n}=\left(c_{1}, \ldots, c_{|\mathcal{X}|}\right) \in \mathbb{R}^{|\mathcal{X}|}$ (which can depend on $n$ ) will be chosen later, and $\mathcal{T}_{n}=$ $\left\{\left(\frac{k_{1}}{n}-c_{1}, \ldots, \frac{k_{|\mathcal{X}|}}{n}-c_{|\mathcal{X}|}\right): k_{1}, \ldots, k_{|\mathcal{X}|} \in \mathbb{N} \cup\{0\}, k_{1}+\right.$ $\left.\cdots+k_{|\mathcal{X}|}=n\right\}$. Moreover, for ease of exposition, let $T_{n}^{-}$ and $T_{n}^{+}$denote versions of the random variable $T_{n}$ with probability distributions $P_{T_{n}}^{-}$and $P_{T_{n}}^{+}$induced by $P_{X}^{\otimes n}$ and $Q_{X}^{\otimes n}$, respectively, such that

$$
\begin{aligned}
& P_{T_{n}}^{-}(t) \triangleq \mathbb{P}\left(T_{n}^{-}=t\right)=\mathbb{P}\left(\hat{P}_{X_{1}^{n}}=t+C_{n} \mid H=0\right), \\
& P_{T_{n}}^{+}(t) \triangleq \mathbb{P}\left(T_{n}^{+}=t\right)=\mathbb{P}\left(\hat{P}_{X_{1}^{n}}=t+C_{n} \mid H=1\right),
\end{aligned}
$$

for all $t \in \mathcal{T}_{n}$. Then, we clearly have

$$
P_{T_{n}}=\frac{1}{2} P_{T_{n}}^{-}+\frac{1}{2} P_{T_{n}}^{+} .
$$

It is straightforward to verify that $T_{n}$ is a sufficient statistic of $X_{1}^{n}$ for performing inference about $H$. So, the ML decoder of $H$ based on $X_{1}^{n}, \hat{H}_{\mathrm{ML}}^{n}\left(X_{1}^{n}\right)$, is a function of $T_{n}$ without loss of generality (see (19)), and we denote it as $\hat{H}_{\mathrm{ML}}^{n}: \mathcal{T}_{n} \rightarrow\{0,1\}, \hat{H}_{\mathrm{ML}}^{n}\left(T_{n}\right)$ with abuse of notation. Thus, we have $P_{\mathrm{ML}}^{(n)}=\mathbb{P}\left(\hat{H}_{\mathrm{ML}}^{n}\left(T_{n}\right) \neq H\right)$, and (23) implies that

$$
\left\|P_{X}^{\otimes n}-Q_{X}^{\otimes n}\right\|_{\mathrm{TV}}=\left\|P_{T_{n}}^{+}-P_{T_{n}}^{-}\right\|_{\mathrm{TV}} .
$$

It therefore suffices to lower bound the right hand side.

Similar to the proof of [43, Lemma 4.2(iii)], observe that

$$
\begin{aligned}
& \left\|\mathbb{E}\left[T_{n}^{+}\right]-\mathbb{E}\left[T_{n}^{-}\right]\right\|_{2}^{2} \\
& =\left\|\sum_{t \in \mathcal{T}_{n}} t\left(P_{T_{n}}^{+}(t)-P_{T_{n}}^{-}(t)\right)\right\|_{2}^{2} \\
& \stackrel{(\mathrm{a})}{=} \sum_{i=1}^{|\mathcal{X}|}\left(\sum_{t \in \mathcal{T}_{n}}\left(\frac{P_{T_{n}}^{+}(t)-P_{T_{n}}^{-}(t)}{\sqrt{P_{T_{n}}(t)}}\right) t_{i} \sqrt{P_{T_{n}}(t)}\right)^{2} \\
& \stackrel{\text { (b) }}{\leq} 4 \underbrace{\left(\frac{1}{4} \sum_{t \in \mathcal{T}_{n}} \frac{\left(P_{T_{n}}^{+}(t)-P_{T_{n}}^{-}(t)\right)^{2}}{P_{T_{n}}(t)}\right)}_{\triangleq \mathrm{LC}\left(P_{T_{n}}^{+} \| P_{T_{n}}^{-}\right)}\left(\sum_{i=1}^{|\mathcal{X}|} \sum_{t \in \mathcal{T}_{n}} t_{i}^{2} P_{T_{n}}(t)\right) \\
& =4 \mathrm{LC}\left(P_{T_{n}}^{+} \| P_{T_{n}}^{-}\right) \mathbb{E}\left[\left\|T_{n}\right\|_{2}^{2}\right] \\
& \stackrel{\text { (c) }}{\leq} 4 \mathbb{E}\left[\left\|T_{n}\right\|_{2}^{2}\right]\left\|P_{T_{n}}^{+}-P_{T_{n}}^{-}\right\|_{\mathrm{TV}} \text {, }
\end{aligned}
$$

where we let $t=\left(t_{1}, \ldots, t_{|\mathcal{X}|}\right)$ in (a), (b) follows from the Cauchy-Schwarz-Bunyakovsky inequality, LC $(\cdot \| \cdot)$ denotes the 
Vincze-Le Cam distance or triangular discrimination between two probability distributions [68], [69], and (c) upper bounds Vincze-Le Cam distance using TV distance (via the observation that $\left|P_{T_{n}}^{+}(t)-P_{T_{n}}^{-}(t)\right| \leq P_{T_{n}}^{+}(t)+P_{T_{n}}^{-}(t)$ for all $\left.t \in \mathcal{T}_{n}\right)$. We note that (82) is precisely a vector version of [43, Lemma 4.2(iii)]. Hence, combining (81) and (83), we get

$$
\left\|P_{X}^{\otimes n}-Q_{X}^{\otimes n}\right\|_{\mathrm{TV}} \geq \frac{\left\|\mathbb{E}\left[T_{n}^{+}\right]-\mathbb{E}\left[T_{n}^{-}\right]\right\|_{2}^{2}}{4 \mathbb{E}\left[\left\|T_{n}\right\|_{2}^{2}\right]} .
$$

We now select the vector $C_{n}$. Since the numerator of the bound in (84) is invariant to the value of $C_{n}$, the best bound of the form (84) is obtained by minimizing the second moment $\mathbb{E}\left[\left\|T_{n}\right\|_{2}^{2}\right]$. Thus, $C_{n}$ is given by

$$
C_{n}=\mathbb{E}\left[\hat{P}_{X_{1}^{n}}\right]=\frac{1}{2} P_{X}+\frac{1}{2} Q_{X},
$$

using the binary hypothesis testing model (17), where we employ the well-known fact that mean-squared error is minimized by the mean (see, e.g., [70, Section 1.7, Example 7.17]). With this choice of $C_{n}$, notice that

$$
\begin{aligned}
\mathbb{E}\left[T_{n}^{-}\right] & =\frac{1}{2} P_{X}-\frac{1}{2} Q_{X}, \\
\mathbb{E}\left[T_{n}^{+}\right] & =\frac{1}{2} Q_{X}-\frac{1}{2} P_{X}, \\
\mathbb{E}\left[\left\|T_{n}\right\|_{2}^{2}\right] & =\sum_{x \in \mathcal{X}} \mathbb{V} \mathbb{R}\left(\hat{P}_{X_{1}^{n}}(x)\right) .
\end{aligned}
$$

Using these expressions, we can simplify the second moment method bound in (84) and obtain the bound in the lemma statement.

We remark that with the choice of $C_{n}$ in (85), (82) can be perceived as a vector version of the HCR bound in statistics [44], [45], where the Vincze-Le Cam distance replaces the usual $\chi^{2}$-divergence.

\section{APPENDIX C \\ PROOF OF LEMMA 5}

Proof. To upper bound the ML decoding probability of error $P_{\mathrm{ML}}^{(n)}$, we combine (23) and Lemma 4 to get

$$
P_{\mathrm{ML}}^{(n)} \leq \frac{1}{2}\left(1-\frac{\left\|P_{X}-Q_{X}\right\|_{2}^{2}}{4 \sum_{x \in \mathcal{X}} \operatorname{VAR}\left(\hat{P}_{X_{1}^{n}}(x)\right)}\right) .
$$

We now compute the right hand side of this bound explicitly. Observe using (85) that for every $x \in \mathcal{X}$,

$$
\begin{aligned}
\mathbb{V A R}\left(\hat{P}_{X_{1}^{n}}(x)\right)= & \frac{1}{n^{2}} \mathbb{E}\left[\left(\sum_{i=1}^{n} \mathbb{1}\left\{X_{i}=x\right\}\right)^{2}\right] \\
& -\left(\frac{P_{X}(x)+Q_{X}(x)}{2}\right)^{2} \\
= & \frac{P_{X}(x)+Q_{X}(x)}{2 n}-\left(\frac{P_{X}(x)+Q_{X}(x)}{2}\right)^{2} \\
& +\frac{1}{n^{2}} \sum_{\substack{1 \leq i, j \leq n \\
i \neq j}} \mathbb{E}\left[\mathbb{1}\left\{X_{i}=x\right\} \mathbb{1}\left\{X_{j}=x\right\}\right]
\end{aligned}
$$

$$
\begin{aligned}
= & \frac{P_{X}(x)+Q_{X}(x)}{2 n}-\left(\frac{P_{X}(x)+Q_{X}(x)}{2}\right)^{2} \\
& +\left(\frac{n-1}{2 n}\right)\left(P_{X}(x)^{2}+Q_{X}(x)^{2}\right) \\
= & \frac{P_{X}(x)\left(1-P_{X}(x)\right)}{2 n} \\
& +\frac{Q_{X}(x)\left(1-Q_{X}(x)\right)}{2 n} \\
& +\frac{\left(P_{X}(x)-Q_{X}(x)\right)^{2}}{4} \\
\leq & \frac{1}{4 n}+\frac{\left(P_{X}(x)-Q_{X}(x)\right)^{2}}{4},
\end{aligned}
$$

where the equalities follow from straightforward algebraic manipulations, and the final inequality holds because $t(1-t) \leq \frac{1}{4}$ for all $t \in[0,1]$. Plugging this inequality into (86) yields

$$
\begin{aligned}
P_{\mathrm{ML}}^{(n)} & \leq \frac{1}{2}\left(1-\frac{\left\|P_{X}-Q_{X}\right\|_{2}^{2}}{\frac{|\mathcal{X}|}{n}+\left\|P_{X}-Q_{X}\right\|_{2}^{2}}\right) \\
& =\frac{|\mathcal{X}|}{2|\mathcal{X}|+2 n\left\|P_{X}-Q_{X}\right\|_{2}^{2}} \\
& \leq \frac{|\mathcal{X}|}{2|\mathcal{X}|+2 n^{2 \epsilon_{n}}},
\end{aligned}
$$

where the final inequality follows from applying (24). This completes the proof.

\section{APPENDIX D \\ PROOF OF LEMMA 6}

Proof.

Part 1: For the convenience of readers unfamiliar with the notion of iterated random maps, we translate the proofs of [52, Theorem 3.1, Proposition 4.1] into information theoretic language. (We also refer readers to [71, Remark III.2], which shows the forward direction.)

Suppose $P_{Z \mid X}$ satisfies Doeblin $\left(Q_{Z}, \eta\right)$. Then, construct the channel $P_{Z \mid X^{\prime}}$ with input alphabet $\mathcal{X} \cup\{$ E $\}$ and output alphabet $\mathcal{Y}$ such that

$$
P_{Z \mid X^{\prime}}(z \mid x)= \begin{cases}\frac{P_{Z \mid X}(z \mid x)-\eta Q_{Z}(z)}{1-\eta}, & \text { for } x \in \mathcal{X} \\ Q_{Z}(z), & \text { for } x=\mathrm{E}\end{cases}
$$

for all $z \in \mathcal{Y}$ and $x \in \mathcal{X} \cup\{\mathrm{E}\}$, where $P_{Z \mid X}(z \mid x)-\eta Q_{Z}(z) \geq$ 0 due to Definition 5, and $\sum_{z \in \mathcal{Y}} P_{Z \mid X}(z \mid x)-\eta Q_{Z}(z)=1-$ $\eta$. It follows via a direct calculation that $P_{Z \mid X}=q-\mathrm{EC}(\eta)$. $P_{Z \mid X^{\prime}}$ (i.e., $P_{Z \mid X}$ is the product of the stochastic matrices $q$-EC $(\eta)$ and $\left.P_{Z \mid X^{\prime}}\right)$, which means that $P_{Z \mid X}$ is a degraded version of $q-\mathrm{EC}(\eta)$.

To prove the reverse direction, suppose $P_{Z \mid X}$ is a degraded version of $q-\mathrm{EC}(\eta)$. Then, using Definition 2, there exists a channel $P_{Z \mid X^{\prime}}$ with input alphabet $\mathcal{X} \cup\{\mathrm{E}\}$ and output alphabet $\mathcal{Y}$ such that $P_{Z \mid X}=q-\mathrm{EC}(\eta) \cdot P_{Z \mid X^{\prime}}$. Hence, it is straightforward to show that for every $x \in \mathcal{X}$ and $y \in \mathcal{Y}$,

$$
\begin{aligned}
P_{Z \mid X}(z \mid x) & =(1-\eta) P_{Z \mid X^{\prime}}(z \mid x)+\eta P_{Z \mid X^{\prime}}(z \mid \mathrm{E}) \\
& \geq \eta P_{Z \mid X^{\prime}}(z \mid \mathrm{E}),
\end{aligned}
$$


where the inequality holds because $(1-\eta) P_{Z \mid X^{\prime}}(z \mid x) \geq 0$. Thus, employing Definition 5, this implies that $P_{Z \mid X}$ satisfies $\operatorname{Doeblin}\left(P_{Z \mid X^{\prime}}(\cdot \mid \mathrm{E}), \eta\right)$. This completes the proof of part 1.

Part 2: We refer readers to [55, Lemma 4] for a proof of this part. (It is worth juxtaposing $\eta_{*}\left(P_{Z \mid X}\right)$ with [72, Equations (58) and (102)], which state that contraction coefficients of operator convex $f$-divergences characterize the extremal erasure probability $\eta$ such that $P_{Z \mid X}$ is dominated by a $q-\mathrm{EC}(\eta)$ in the "less noisy" sense; see [72] for details.)

\section{REFERENCES}

[1] A. Makur, "Bounds on permutation channel capacity," in Proceedings of the IEEE International Symposium on Information Theory (ISIT), Los Angeles, CA, USA, June 21-26 2020, pp. 1-6.

[2] A. Makur, "Information capacity of BSC and BEC permutation channels," in Proceedings of the 56th Annual Allerton Conference on Communication, Control, and Computing, Monticello, IL, USA, October 2-5 2018, pp. 1112-1119.

[3] S. N. Diggavi and M. Grossglauser, "On transmission over deletion channels," in Proceedings of the 39th Annual Allerton Conference on Communication, Control, and Computing, Monticello, IL, USA, October 3-5 2001, pp. 573-582.

[4] M. Mitzenmacher, "Polynomial time low-density parity-check codes with rates very close to the capacity of the $q$-ary random deletion channel for large q," IEEE Transactions on Information Theory, vol. 52, no. 12, pp. 5496-5501, December 2006

[5] J. J. Metzner, "Simplification of packet-symbol decoding with errors, deletions, misordering of packets, and no sequence numbers," IEEE Transactions on Information Theory, vol. 55, no. 6, pp. 2626-2639, June 2009.

[6] S. Kudekar, S. Kumar, M. Mondelli, H. D. Pfister, E. Şaşoğlu, and R. L. Urbanke, "Reed-Muller codes achieve capacity on erasure channels," IEEE Transactions on Information Theory, vol. 63, no. 7, pp. 42984316, July 2017.

[7] Y. Xu and T. Zhang, "Variable shortened-and-punctured Reed-Solomon codes for packet loss protection," IEEE Transactions on Broadcasting, vol. 48, no. 3, pp. 237-245, September 2002.

[8] M. Gadouleau and A. Goupil, "Binary codes for packet error and packet loss correction in store and forward," in Proceedings of the International ITG Conference on Source and Channel Coding (SCC), no. 25, Siegen, Germany, January 18-21 2010, pp. 1-6.

[9] J. M. Walsh, S. Weber, and C. wa Maina, "Optimal rate-delay tradeoffs and delay mitigating codes for multipath routed and network coded networks," IEEE Transactions on Information Theory, vol. 55, no. 12, pp. 5491-5510, December 2009.

[10] M. Kovačević and D. Vukobratović, "Subset codes for packet networks," IEEE Communications Letters, vol. 17, no. 4, pp. 729-732, April 2013.

[11] M. Kovačević and D. Vukobratović, "Perfect codes in the discrete simplex," Designs, Codes and Cryptography, vol. 75, no. 1, pp. 81-95, April 2015.

[12] M. Kovačević and V. Y. F. Tan, "Codes in the space of multisetsCoding for permutation channels with impairments," IEEE Transactions on Information Theory, vol. 64, no. 7, pp. 5156-5169, July 2018.

[13] S. M. H. T. Yazdi, H. M. Kiah, E. Garcia-Ruiz, J. Ma, H. Zhao, and O. Milenkovic, "DNA-based storage: Trends and methods," IEEE Transactions on Molecular, Biological, and Multi-Scale Communications, vol. 1, no. 3, pp. 230-248, September 2015.

[14] H. M. Kiah, G. J. Puleo, and O. Milenkovic, "Codes for DNA sequence profiles," IEEE Transactions on Information Theory, vol. 62, no. 6, pp. 3125-3146, June 2016.

[15] R. Heckel, I. Shomorony, K. Ramchandran, and D. N. C. Tse, "Fundamental limits of DNA storage systems," in Proceedings of the IEEE International Symposium on Information Theory (ISIT), Aachen, Germany, June 25-30 2017, pp. 3130-3134.

[16] M. Kovačević and V. Y. F. Tan, "Asymptotically optimal codes correcting fixed-length duplication errors in DNA storage systems," IEEE Coтmunications Letters, vol. 22, no. 11, pp. 2194-2197, November 2018.

[17] I. Shomorony and R. Heckel, "Capacity results for the noisy shuffling channel," in Proceedings of the IEEE International Symposium on Information Theory (ISIT), Paris, France, July 7-12 2019, pp. 762-766.

[18] T. M. Cover and J. A. Thomas, Elements of Information Theory, 2nd ed. Hoboken, NJ, USA: John Wiley \& Sons, Inc., 2006.
[19] Y. Polyanskiy and Y. Wu, "Lecture notes on information theory," May 2019, Department of Electrical Engineering and Computer Science, MIT, Cambridge, MA, USA, Lecture Notes 6.441.

[20] O. Kosut and L. Sankar, "New results on third-order coding rate for universal fixed-to-variable source coding," in Proceedings of the IEEE International Symposium on Information Theory (ISIT), Honolulu, HI, USA, June 29-July 4 2014, pp. 2689-2693.

[21] T. M. Cover, "Broadcast channels," IEEE Transactions on Information Theory, vol. IT-18, no. 1, pp. 2-14, January 1972.

[22] P. P. Bergmans, "Random coding theorem for broadcast channels with degraded components," IEEE Transactions on Information Theory, vol IT-19, no. 2, pp. 197-207, March 1973.

[23] A. El Gamal and Y.-H. Kim, Network Information Theory. New York, NY, USA: Cambridge University Press, 2011.

[24] D. Blackwell, "Comparison of experiments," in Proceedings of the Second Berkeley Symposium on Mathematical Statistics and Probability (Berkeley, CA, USA, July 31-August 12 1950), J. Neyman, Ed. Berkeley, CA, USA: University of California Press, 1951, pp. 93-102.

[25] S. Sherman, "On a theorem of Hardy, Littlewood, Polya, and Blackwell," Proceedings of the National Academy of Sciences of the United States of America (PNAS), vol. 37, no. 12, pp. 826-831, December 1951.

[26] C. Stein, "Notes on a seminar on theoretical statistics. I. Comparison of experiments," University of Chicago, Tech. Rep., 1951.

[27] M. Leshno and Y. Spector, "An elementary proof of Blackwell's theorem," Mathematical Social Sciences, Elsevier, vol. 25, no. 1, pp. 95-98, December 1992.

[28] E. Torgersen, "Stochastic orders and comparison of experiments," in Stochastic Orders and Decision Under Risk, ser. Lecture NotesMonograph Series, K. Mosler and M. Scarsini, Eds., vol. 19. Hayward, CA, USA: Institute of Mathematical Statistics, 1991, pp. 334-371.

[29] E. Torgersen, Comparison of Statistical Experiments, ser. Encyclopedia of Mathematics and Its Applications. New York, NY, USA: Cambridge University Press, 1991.

[30] T. Richardson and R. Urbanke, Modern Coding Theory. Cambridge, UK: Cambridge University Press, 2008.

[31] A. W. Marshall, I. Olkin, and B. C. Arnold, Inequalities: Theory of Majorization and Its Applications, 2nd ed., ser. Springer Series in Statistics. New York, NY, USA: Springer, 2011.

[32] G. Dahl, "Matrix majorization," Linear Algebra and its Applications, Elsevier, vol. 288, pp. 53-73, February 1999.

[33] G. Dahl, "Majorization polytopes," Linear Algebra and its Applications, Elsevier, vol. 297, pp. 157-175, August 1999.

[34] A. Makur, "Information contraction and decomposition," Sc.D. Thesis in Electrical Engineering and Computer Science, Massachusetts Institute of Technology, Cambridge, MA, USA, May 2019.

[35] A. Makur and Y. Polyanskiy, "Comparison of channels: Criteria for domination by a symmetric channel," IEEE Transactions on Information Theory, vol. 64, no. 8, pp. 5704-5725, August 2018.

[36] A. Makur and Y. Polyanskiy, "Less noisy domination by symmetric channels," in Proceedings of the IEEE International Symposium on Information Theory (ISIT), Aachen, Germany, June 25-30 2017, pp. 2463-2467.

[37] E. Mossel, K. Oleszkiewicz, and A. Sen, "On reverse hypercontractivity," Geometric and Functional Analysis, vol. 23, no. 3, pp. 1062-1097, June 2013.

[38] J. A. Adell, A. Lekuona, and Y. Yu, "Sharp bounds on the entropy of the Poisson law and related quantities," IEEE Transactions on Information Theory, vol. 56, no. 5, pp. 2299-2306, May 2010.

[39] W. Hoeffding, "Probability inequalities for sums of bounded random variables," Journal of the American Statistical Association, vol. 58, no. 301, pp. 13-30, March 1963.

[40] G. W. Wornell, "Inference and information," May 2017, Department of Electrical Engineering and Computer Science, MIT, Cambridge, MA, USA, Lecture Notes 6.437

[41] A. B. Tsybakov, Introduction to Nonparametric Estimation, ser. Springer Series in Statistics. New York, NY, USA: Springer, 2009.

[42] D. A. Levin, Y. Peres, and E. L. Wilmer, Markov Chains and Mixing Times, 1st ed. Providence, RI, USA: American Mathematical Society, 2009.

[43] W. Evans, C. Kenyon, Y. Peres, and L. J. Schulman, "Broadcasting on trees and the Ising model," The Annals of Applied Probability, vol. 10, no. 2, pp. 410-433, May 2000.

[44] J. M. Hammersley, "On estimating restricted parameters," Journal of the Royal Statistical Society, Series B (Methodological), vol. 12, no. 2, pp. 192-240, 1950. 
[45] D. G. Chapman and H. Robbins, "Minimum variance estimation without regularity assumptions," The Annals of Mathematical Statistics, vol. 22, no. 4, pp. 581-586, December 1951.

[46] R. A. Horn and C. R. Johnson, Topics in Matrix Analysis. New York, NY, USA: Cambridge University Press, 1991.

[47] V. Rakočević and H. K. Wimmer, "A variational characterization of canonical angles between subspaces," Journal of Geometry, vol. 78 , no. 1, pp. 122-124, 2003.

[48] M. Bloch and J. Barros, Physical-Layer Security: From Information Theory to Security Engineering. New York, NY, USA: Cambridge University Press, 2011.

[49] R. A. Horn and C. R. Johnson, Matrix Analysis, 2nd ed. New York, NY, USA: Cambridge University Press, 2013.

[50] I. Sason and S. Shamai, Performance Analysis of Linear Codes under Maximum-Likelihood Decoding: A Tutorial, ser. Foundations and Trends in Communications and Information Theory, S. Verdú, Ed. Hanover, MA, USA: now Publishers Inc., 2006, vol. 3, no. 1-2.

[51] R. W. Keener, Theoretical Statistics: Topics for a Core Course, ser. Springer Texts in Statistics. New York, NY, USA: Springer, 2010.

[52] R. Bhattacharya and E. C. Waymire, "Iterated random maps and some classes of Markov processes," in Stochastic Processes: Theory and Methods, ser. Handbook of Statistics, D. N. Shanbhag and C. R. Rao, Eds., vol. 19. Amsterdam, Netherlands: North-Holland, Elsevier, 2001, pp. $145-170$.

[53] W. Doeblin, "Sur les proprietes asymptotiques de mouvement régis par certains types de chaînes simples," Bulletin Mathématique de la Société Roumaine des Sciences, vol. 39, no. 1, pp. 57-115, 1937, in French.

[54] W. Doeblin, "Exposé de la théorie des chaînes simples constantes de Markov à un nombre fini d'états," Revue Mathématique de l'Union Interbalkanique, vol. 2, pp. 77-105, 1938, in French.

[55] A. Gohari, O. Günlü, and G. Kramer, "Coding for positive rate in the source model key agreement problem,” May 2019, arXiv:1709.05174v5 [cs.IT].

[56] J. E. Cohen, Y. Iwasa, G. Rautu, M. B. Ruskai, E. Seneta, and G. Zbăganu, "Relative entropy under mappings by stochastic matrices," Linear Algebra and its Applications, Elsevier, vol. 179, pp. 211-235, January 1993.

[57] K. B. Athreya and P. Ney, "A new approach to the limit theory of recurrent Markov chains," Transactions of the American Mathematical Society, vol. 245, pp. 493-501, November 1978.

[58] E. Nummelin, "A splitting technique for Harris recurrent Markov chains," Zeitschrift für Wahrscheinlichkeitstheorie und Verwandte Gebiete, vol. 43, no. 4, pp. 309-318, December 1978.

[59] G. Grimmett, "Percolation and disordered systems," in Lectures on Probability Theory and Statistics: Ecole d'Eté de Probabilités de SaintFlour XXVI-1996, ser. Lecture Notes in Mathematics, P. Bernard, Ed. vol. 1665. Berlin, Heidelberg, Germany: Springer, 1997, pp. 153-300.

[60] T. Feder, "Reliable computation by networks in the presence of noise," IEEE Transactions on Information Theory, vol. 35, no. 3, pp. 569-571, May 1989.

[61] A. Makur, E. Mossel, and Y. Polyanskiy, "Broadcasting on random directed acyclic graphs," IEEE Transactions on Information Theory, vol. 66, no. 2, pp. 780-812, February 2020.

[62] A. Makur, E. Mossel, and Y. Polyanskiy, "Broadcasting on random networks," in Proceedings of the IEEE International Symposium on Information Theory (ISIT), Paris, France, July 7-12 2019, pp. 1632 1636.

[63] C. E. Shannon, "The zero error capacity of a noisy channel," IRE Transactions on Information Theory, vol. 2, no. 3, pp. 8-19, September 1956.

[64] L. Lovász, "On the Shannon capacity of a graph,” IEEE Transactions on Information Theory, vol. IT-25, no. 1, pp. 1-7, January 1979.

[65] R. G. Gallager, Information Theory and Reliable Communication. New York, NY, USA: John Wiley \& Sons, Inc., 1968.

[66] Y. Polyanskiy, H. V. Poor, and S. Verdú, "Channel coding rate in the finite blocklength regime," IEEE Transactions on Information Theory, vol. 56, no. 5, pp. 2307-2359, May 2010.

[67] V. Y. F. Tan, Asymptotic Estimates in Information Theory with NonVanishing Error Probabilities, ser. Foundations and Trends in Communications and Information Theory, S. Verdú, Ed. Hanover, MA, USA: now Publishers Inc., 2014, vol. 11, no. 1-2.

[68] I. Vincze, "On the concept and measure of information contained in an observation," in Contributions to Probability: A Collection of Papers Dedicated to Eugene Lukacs, J. Gani and V. K. Rohatgi, Eds. New York, NY, USA: Academic Press, 1981, pp. 207-214.

[69] L. Le Cam, Asymptotic Methods in Statistical Decision Theory, ser Springer Series in Statistics. New York, NY, USA: Springer, 1986.
[70] E. L. Lehmann and G. Casella, Theory of Point Estimation, 2nd ed., ser. Springer Texts in Statistics. New York, NY, USA: Springer, 1998.

[71] M. Raginsky, "Strong data processing inequalities and $\Phi$-Sobolev inequalities for discrete channels," IEEE Transactions on Information Theory, vol. 62, no. 6, pp. 3355-3389, June 2016.

[72] A. Makur and L. Zheng, "Comparison of contraction coefficients for $f$-divergences," Problems of Information Transmission, vol. 56, no. 2, pp. 103-156, April 2020. 